\title{
Single-cell transcriptome profiling reveals neutrophil heterogeneity and orchestrated maturation during homeostasis and bacterial infection
}

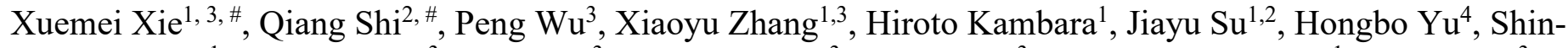 \\ Young Park ${ }^{1}$, Rongxia Guo ${ }^{3}$, Qian Ren ${ }^{3}$, Sudong Zhang, ${ }^{3}$, Yuanfu Xu ${ }^{3}$, Leslie E. Silberstein ${ }^{1}$, Tao Cheng ${ }^{3}$, \\ Fengxia $\mathrm{Ma}^{3, *}$, Cheng $\mathrm{Li}^{2}{ }^{2}$ and Hongbo R. Luo ${ }^{1, *}$ \\ ${ }^{1}$ Department of Pathology, Harvard Medical School; \\ Department of Lab Medicine, The Stem Cell Program, Children's Hospital Boston; \\ Dana-Farber/Harvard Cancer Center \\ Boston MA 02115 USA
}

${ }^{2}$ School of Life Sciences, Center for Bioinformatics, Center for Statistical Science, Peking University, Beijing, 100871, China

${ }^{3}$ The State Key Laboratory of Experimental Hematology, Institute of Hematology and Blood Diseases Hospital, Chinese Academy of Medical Sciences and Peking Union Medical College, 288 Nanjing Road, Tianjin, 300020, China

\author{
${ }^{4}$ VA Boston Healthcare System, Department of Pathology and Laboratory Medicine, 1400 VFW Parkway \\ West Roxbury, MA 02132 USA \\ \# These authors contributed equally to this work. \\ * To whom all correspondence should be addressed. \\ Enders Research Building, Room 811 \\ Boston, MA 02115, USA
}

Hongbo R. Luo: Hongbo.Luo@childrens.harvard.edu

(Lead Contact) Phone: 617-919-2303

Cheng Li: $\quad$ cheng_li@pku.edu.cn

Fengxia Ma: mafengxia@ihcams.ac.cn

Running title: A single-cell resolution neutrophil transcriptional landscape

Keywords: Neutrophil, single-cell, transcriptome, granulopoiesis, heterogeneity, maturation, innate immunity infection. 


\section{Highlights}

- A comprehensive single-cell resolution transcriptional landscape of mouse neutrophil maturation and fate decision under steady-state and bacterial infection conditions.

- The pathogen clearance machinery in neutrophils is continuously and gradually built during neutrophil differentiation, maturation, and aging, driven by both known and uncharacterized transcription factors.

- The three mature neutrophil subsets in peripheral blood, including a novel ISG-expressing subset, are derived from distinct bone marrow neutrophil precursors.

- Bacterial infection reprograms the genetic architecture of neutrophil populations, alters dynamic transition between each subpopulation, and primes neutrophils for augmented functionality without affecting overall neutrophil heterogeneity.

- Bacterial infection-induced emergency granulopoiesis is mediated by augmented proliferation of early stage neutrophil progenitors and accelerated post-mitotic maturation. 


\section{Summary}

The full neutrophil heterogeneity and differentiation landscape remains incompletely characterized. Here we profiled $>25,000$ differentiating and mature mouse neutrophils using single-cell RNA sequencing to provide a comprehensive transcriptional landscape of neutrophil maturation, function, and fate decision in their steady state and during bacterial infection. Eight neutrophil populations were defined by distinct molecular signatures. The three mature peripheral blood neutrophil subsets arise from distinct maturing bone marrow neutrophil subsets. Driven by both known and uncharacterized transcription factors, neutrophils gradually acquire microbicidal capability as they traverse the transcriptional landscape, representing an evolved mechanism for fine-tuned regulation of an effective but balanced neutrophil response. Bacterial infection reprograms the genetic architecture of neutrophil populations, alters dynamic transition between each subpopulation, and primes neutrophils for augmented functionality without affecting overall heterogeneity. In summary, these data establish a reference model and general framework for studying neutrophil-related disease mechanisms, biomarkers, and therapeutic targets at single-cell resolution. 


\section{Introduction}

Neutrophils migrate from the circulating blood to infected tissues in response to inflammatory stimuli, where they protect the host by phagocytosing, killing, and digesting bacterial and fungal pathogens (Cossio et al., 2019; Darrah and Andrade, 2012; Lee et al., 2003; Ley et al., 2018; Nauseef and Borregaard, 2014; NicolasAvila et al., 2017; Segal, 2005). Neutrophil function must be tightly controlled during infection and inflammation: excessive neutrophil accumulation or hyper-responsiveness can be detrimental (Baggiolini, 2001; Castanheira and Kubes, 2019; Davis et al., 2003; Wipke and Allen, 2001), while defects in neutrophil development, trafficking, or function can result in immunological and hematological disorders (Bunting et al., 2002; Burg and Pillinger, 2001; Dinauer, 2019; Fodil et al., 2016; Kruger et al., 2015; Witko-Sarsat et al., 2000).

In adults, neutrophils are mainly produced in the bone marrow (BM) through stepwise progression of myeloid progenitors (Cowland and Borregaard, 2016a; Lawrence et al., 2018; Ward et al., 2000). Neutrophil maturation typically follows five main morphological stages: myeloblasts (MBs), promyelocytes (PMs), myelocytes (MCs), metamyelocytes (MMs), band neutrophils and segmented neutrophils (BC/SCs). Cell division is halted at an early stage of neutrophil differentiation (Klausen et al., 2004; Mora-Jensen et al., 2011), while post-mitotic maturation after the last cell division occurs in the BM, with mature neutrophils released through the venular endothelium into the bloodstream as non-dividing polymorphonuclear neutrophils (PMNs) (Cowland and Borregaard, 2016a).

Neutrophil populations are not, however, homogenous (Adrover et al., 2016; Ley et al., 2018; $\mathrm{Ng}$ et al., 2019; Nicolas-Avila et al., 2017; Scapini et al., 2016; Silvestre-Roig et al., 2016; Yvan-Charvet and Ng, 2019): differentiation and maturation produce distinct neutrophils subpopulations which may be pre-programmed with different functions; discrete microenvironments can modify neutrophil function and behavior; and rapid neutrophil aging, their short lifespan, and mechanically-induced cellular responses as they enter and exit capillaries (Doerschuk, 2000; Wang and Doerschuk, 2002) contribute to neutrophil heterogeneity. Neutrophil classification has traditionally relied on morphology, surface marker expression, or gradient separation, which while simple and robust do not capture the full neutrophil compartment repertoire. Some neutrophil subpopulations overlap, making nomenclature confusing and contributing to controversies regarding neutrophil function and ontogeny. In addition, the exact function of some neutrophil subpopulations and the molecular bases of heterogeneity are varied and remain elusive.

Single-cell RNA sequencing (scRNA-seq) is a powerful tool to explore immune cell heterogeneity (Adlung and Amit, 2018; Papalexi and Satija, 2018; Stubbington et al., 2017). Here we adopt an unbiased, systematic approach to dissect mouse neutrophil populations in the bone marrow, peripheral blood, and spleen at singlecell resolution. In doing so, we provide the first comprehensive reference map of differentiating and mature 
neutrophil transcriptional states in both healthy and E. coli-infected hosts. Our analysis addresses critical deficiencies in our understanding of neutrophil biology, and the resource will provide the wider community with new opportunities to further dissect the complex molecular regulation of neutrophil maturation, function, and fate, including for therapeutic impact.

\section{Results}

\section{Mouse neutrophil atlas in the steady state}

To characterize the gene expression programs dictating neutrophil differentiation, maturation and steady-state heterogeneity, we obtained a comprehensive scRNA-seq map of mouse neutrophils under steady-state conditions. $\mathrm{Gr}^{+}$cells were isolated from the bone marrow (BM-Gr1), peripheral blood (PB-Gr1), and spleen (SP-Gr1) by FACS (Fig.1a). To capture the whole spectrum of neutrophil maturation and identify potential neutrophil populations with lower Gr1 antigen (mainly Ly6G) expression, we also included Gr1 ${ }^{\text {low }}$ and a few $\mathrm{Gr1}^{-}$cells in each sample. In addition, to gain insights into granulopoiesis in its entirety, we included a sample enriched for $\mathrm{c}-\mathrm{Kit}^{+} \mathrm{BM}$ HSPCs mixed with BM-Gr1 cells at a 2:3 ratio to artificially create a BM-cKit/Gr1 population. The sorted high-quality intact single cells (Fig.S1a-e) were processed for RNA-seq using the 10x Genomics Chromium platform (Fig.1a).

To obtain a harmonized atlas, we performed an integrated analysis of cells pooled from different organs (Fig.S1f) and projected into two dimensions with Uniform Manifold Approximation and Projection (UMAP) (Becht et al., 2018a) based on their transcriptomic profiles. Unbiased, graph-based clustering identified seven major cell populations (Fig.S1g-I and Table S2). Lymphocytes and monocytes from different samples overlapped in the same defined clusters, indicating that there was little batch effect between samples. Since the majority of the cells were $\mathrm{Gr}^{+}$, the largest cell cluster identified by this analysis was the neutrophil population, as intended (Fig.S1g).

To dissect neutrophil heterogeneity, we examined the neutrophil-related populations (MPs and neutrophils). Unsupervised clustering partitioned differentiating and mature neutrophils into eight clusters (G0-4; G5a-c;

Fig.1b), most containing cells derived from multiple tissues with overlapping UMAP coordinates. G0-4 mainly originated from the BM and represented neutrophils differentiating in the BM, while G5a-c mainly originated from peripheral tissues (Fig.1c). There was substantial differential gene expression between the groups (Fig.1d and Table S3). For quality assurance, we merged our raw data with another high-quality published dataset (Giladi et al., 2018) (Fig.S1j-k), which agreed with our data well, our data detecting even greater numbers of genes (Fig.S1k). We identified 1,098 differentially-expressed genes (DEGs) and 27 signature genes that distinguished each subpopulation (Fig.1e). In a gene ontology analysis of DEGs (Fig.1f), cell cycle-related 
genes were as expected highly expressed in earlier phases of neutrophil maturation (G0, G1, and G2). G0 and G1 cells were also enriched for protein synthesis genes related to rRNA processing and protein translation. Interestingly, genes related to cytokine production started to be expressed in dividing G2 cells.

\section{Neutrophil differentiation and maturation trajectories}

Since we intentionally included c-Kit ${ }^{+}$cells, significant numbers of CD34 ${ }^{+} \mathrm{G} 0$ cells were detected. According to known gene signatures (Karamitros et al., 2018; Nestorowa et al., 2016; Paul et al., 2015; Velten et al., 2017), we concluded that the G0 population mainly consisted of granulocyte-macrophage progenitors (GMPs) expressing typical genes such as Cd117 (Kit), Cd34, and Sox4 and neutrophil primary granule genes such as Mpo, Elane, and Prtn3 (Fig.1e). To investigate the interrelationship between neutrophil subpopulations, we conducted hierarchical clustering (Fig.1g). Consistent with UMAP clustering, neutrophils in the PB (G5a, G5b, and G5c) were closely associated but more remote from BM cells (G1-4). Using Monocle (Qiu et al., 2017a) to place differentiating neutrophil populations along possible granulopoiesis trajectories in pseudo-time (Fig.1h), neutrophil differentiation and maturation occurred on a tightly organized trajectory, starting from G1 cells in the $\mathrm{BM}$ and ending with G5 cells in the PB and the spleen. G1 to G2 cells underwent active proliferation, with cell division stopping abruptly thereafter (Fig.1i). A cluster of G3 cells followed G2 expansion and expressed secondary granule genes such as Ltf, Camp, and Ngp (Fig.1e). Neutrophil differentiation in the BM concluded with a more mature G4 population highly expressing Mmp8, a key granule protein for neutrophil-mediated host defenses, and Ccl6 which is important for neutrophil mobilization (Fig.1d-e). Finally, we measured the maturation score of each differentiating neutrophil population based on the expression of genes related to neutrophil differentiation and maturation (Table S4). G5 cells, which were mainly in the PB and peripheral tissues, were the most mature neutrophils, while G4 cells showed the highest maturation score among BM neutrophils (Fig.1j).

\section{A "sorting mechanism" for generating heterogeneous neutrophil granules}

A well-accepted mechanism explaining neutrophil granule heterogeneity is "targeting by timing of biosynthesis" (Borregaard and Cowland, 1997; Borregaard et al., 2007; Cowland and Borregaard, 2016a), i.e., granule proteins synthesized at the same time in developing neutrophils will end up in the same granule without granule type-specific sorting. We examined the expression of various granule genes in differentiating neutrophils. Lactoferrin-positive granules are often defined as specific (secondary) granules, while lactoferrinnegative but gelatinase-positive granules are known as gelatinase (tertiary) granules. As expected, a major antibacterial specific granule protein, CAP-18 (Camp product) (Sorensen et al., 1997), was expressed in G2 and G3 cells when specific granules were formed. Surprisingly, this protein, which is not in the tertiary granules and secretary vesicles, was also expressed in G4 neutrophils containing tertiary granules and secretary vesicles. Similarly, another specific granule protein, NGAL (Lcn2 product), which co-localizes completely with 
lactoferrin (Kjeldsen et al., 1994), was also highly expressed in G4 neutrophils (Fig.2a). Thus, "targeting by timing of biosynthesis" may not explain all granule heterogeneity, and some granule proteins must be tagged to direct them to particular granules.

Collagenase (Mmp8 product), a key innate immunity and inflammation enzyme, was thought to be localized exclusively in specific granules (Murphy et al., 1977) and co-expressed with lactoferrin. However, at single-cell level, collagenase was always co-expressed with gelatinase, with the highest expression detected in G4 neutrophils and almost no expression in G2 cells highly expressing lactoferrin (Fig.2a), suggesting that collagenase should be considered a gelatinase rather than a specific granule protein.

\section{scRNA-seq-morphology correlates}

We next compared scRNA-seq-defined neutrophil populations with the classic morphology-defined neutrophils. MBs, PMs, MCs, MMs, and mature neutrophils (including BCs and SCs) were isolated by FACS based on c-Kit and Ly6g expression (Satake et al., 2012), their identities confirmed morphologically (Fig.2d), and bulk RNAseq performed. Most of the molecular signatures identified by scRNA-seq were also detected in bulk RNA-seq data (Fig.2e and Fig.S2a). To further dissect morphological heterogeneity, a regression-based deconvolution approach was applied on bulk expression profiles. MB were a mixture of G0/G1, PM were G1, MC were G1/G2, MM were G2, and BC/SC were G3/G4 (Fig.2f). MBs highly expressed the stem cell marker Cd34 and translation-related genes such as Eef1a1, while the highest expression of primary granule-related genes was detected in the PM population (Fig.S2a-b). MCs and MMs were highly proliferative neutrophils expressing high levels of cell cycle-related genes, which were significantly downregulated in mature neutrophils (Fig.S2c). $\mathrm{BC} / \mathrm{SC}$ neutrophils expressed both G3 and G4 signature genes, confirming that this population is a mixture of G3 and G4 cells (Fig.S2a).

\section{The PB and spleen contain three distinct neutrophil subpopulations}

Three major neutrophil subpopulations were identified in the PB and spleen with 172 DEGs (Table S3, Fig.2g). Overall, BM and PB neutrophils were very different, implicating the microenvironment in driving neutrophil transcription. Similar to G4 BM cells, G5a cells highly expressed Mmp8 and S100a8 (Fig.2g) and genes related to neutrophil migration and inflammatory responses (Fig.2h). Interestingly, a group of G5b neutrophils expressed a set of interferon-stimulated genes (ISGs) such as Ifit3 and Isg15 (Fig.S2d). Although G5b appears to be a separate mature neutrophil subpopulation, they are not interferon-stimulated: unchallenged mice do not produce interferons, and unlike true interferon-induced ISG expression, many ISGs were not upregulated in the G5b population (e.g., Irf3, Ifitm6) (Fig.S2d).

Trajectory analysis showed that G5a and G5b neutrophils gradually developed into G5c neutrophils (Fig.S2e) the latter showing the highest aging score (Fig.2i-j). Aging is a main mechanism that accounts for neutrophil 
heterogeneity (Adrover et al., 2016): aged neutrophils are smaller with fewer granules and granular multi-lobed nuclei, produce more NETs, and migrate out of capillaries less efficiently (Casanova-Acebes et al., 2013; Doerschuk et al., 1994; Kolaczkowska et al., 2015; Tanji-Matsuba et al., 1998). By applying a two-component Gaussian mixture model, we further identified $15 \%$ of G5c neutrophils as aged, significantly higher than in G5b or G5a populations (Fig.2j). Although G5c cells appeared to more aged, the mitochondrial UMI percentage was not elevated in G5c cells, indicating continued viability in the PB and spleen (Fig.S2f). G5c marker genes were significantly enriched for ribosome biogenesis, cytoplasmic translation, post-transcriptional regulation, and LPS-mediated signaling pathways by GO analysis (Fig.2h). These results agree with recent studies showing that aged neutrophils are highly functional and may exhibit a higher phagocytic activity than non-aged neutrophils (Kolaczkowska, 2016; Uhl et al., 2016).

\section{The pathogen clearance machinery is continuously and gradually built during neutrophil differentiation, maturation, and aging}

We next assessed the expression of genes critical to several typical neutrophil-mediated microbial clearance functions. Phagocytosis, chemotaxis, and neutrophil activation scores increased drastically during the early stages of granulopoiesis, peaked at G3, and remained relatively stable thereafter (Fig.S3a-c). In neutrophils, phagocytic NADPH oxidase is responsible for pathogen-induced ROS production and ROS-mediated pathogen killing. Similarly, "NADPH oxidase score", calculated based on the expression of the seven NADPH oxidaserelated genes, increased during G0 to G1 and G2 transition, peaked at G3, and then decreased by $20 \%$ in mature neutrophils (Fig.S3d). However, the dynamics of the oxidase complex subunits varied through neutrophil differentiation (Fig.S3e): Cybb (gp91-phox) was maximally expressed in G2 and G3 cells and abruptly downregulated thereafter; and Ncf2 was upregulated in G3, peaked in G4 cells, and then remained stable. Sequential subunit expression ensures maximal stimulation-triggered NADPH oxidase activation at the later stages of neutrophil maturation and minimal activation in immature neutrophils in the BM. Collectively, this tightly orchestrated neutrophil maturation program ensures that maximum neutrophil activation only occurs when neutrophils are fully mature and mobilized to the PB so that innate host defense execution is effective and safe. Notably, genes related to mitochondria-mediated ROS production were significantly downregulated during neutrophil maturation, further supporting that neutrophil ROS production is mainly mediated by phagocytic NADPH oxidase (Fig.S3f).

Mature neutrophils derive energy mainly from glycolysis (Borregaard and Herlin, 1982). However, metabolismrelated genes (Fig.S3g), including those related to glycolysis (Fig.S3h) were downregulated in mature neutrophils. Similarly, genes related to glucose transportation were also not upregulated in mature neutrophils (Fig.S3i). These data suggest that glycolysis-dominant metabolism in neutrophils is likely to be driven by posttranscriptional or/and post-translational mechanisms rather than transcriptional upregulation of related genes. 


\section{Organ-specific transcriptome features}

Compared to early-stage maturing BM neutrophils, most neutrophils in the PB and SP were a mature G5 population with similar gene expression patterns (Fig.S3j). PB neutrophils expressed chemokines (e.g., Il1b), ribosomal genes (e.g., Rps27rt), and genes related to neutrophil activation and function (e.g., Csf3r, Fgl2). Notably, SP neutrophils highly expressed the transcription factors Fos and Junb, chemokine (C-X-C motif) ligand 2 (Cxcl2/Mip2a), HLA class II histocompatibility antigen gamma chain (Cd74), and certain myeloid transcription factors (e.g., Cebpb) (Fig.S3j). KEGG enrichment analysis revealed PB neutrophils were enriched for genes related to malaria and African trypanosomiasis, while SP neutrophils were more enriched for genes related to leishmaniasis and Yersinia infection, suggesting that PB and SP neutrophils may play distinct roles in combating different infections (Fig.S3k). SP neutrophils also expressed T cell differentiation, IL-17 signaling, TNF signaling, and antigen processing and presentation-related genes, suggesting a role for splenic neutrophils in adaptive immunity. Indeed, a recent study indicated that neutrophils can adopt some APC function (Vono et al., 2017), and splenic B cell-helper neutrophils participate in immunoglobulin diversification and production in the marginal zone (Puga et al., 2011).

\section{Unexpected complexity in neutrophil mobilization}

To further elucidate the relationship between the new neutrophil subpopulations, we traced cell fate and reconstructed cell lineage direction using the recently developed RNA 'velocity' approach (La Manno et al., 2018b) (Fig.3a). Consistent with Monocle (Fig.1h), BM maturation (from G2 to G4) followed a single main branch without significant division, with G3 bearing long vectors and indicating a strong tendency to progress to G4 (Fig.3a). Neutrophils are thought to mobilize from the BM to the PB only at full maturity. Interestingly, the trajectory of a significant number of G3 neutrophils was toward the peripheral G5a population, suggesting mobilization of G3 cells to the PB or tissue without first undergoing full G4 maturation (Fig.3a). BM G4 population split into: (i) the peripheral G5a population, and (ii) the ISG-related G5b population without entering the G5a stage. Thus, although G5a and G5b were most similar (Fig.1g), they are two separate and independent PB neutrophil populations, with G5a derived from BM G3 and G4 cells and G5b derived solely from G4 cells. G5a to G5b conversion was rarely detected in the PB (Fig.3a). Lastly, G5c cells were firmly at the end of neutrophil maturation and differentiation, showing the highest apoptosis scores (Fig.3b) and proportion of apoptotic cells ( $\sim 30 \%$; Fig.3c). There was also significant apoptosis in G5a and G5b cells (Fig.3c), suggesting that death programs can be independent of maturation.

A small number of immature neutrophils also circulate, which are thought to be derived from accidental release of cells closest to maturation (Broxmeyer, 2008; Furze and Rankin, 2008; Suratt et al., 2001). Both the G3 and G4 populations are differentiating neutrophils that mainly exist in the BM. However, we only detected G3 cells in the periphery of healthy mice (5\% of PB and $6 \%$ of SP neutrophils; Fig.1c). During BM granulopoiesis, only 
G3 neutrophils seem to be able to escape from the BM niche, migrate into $\mathrm{PB}$, and travel to other organs. PB and BM G3 cells consistently overlapped on velocity analysis, with some falling into the PB G5a cluster (Fig.3d). Further, PB G3 cells directly differentiated into G5a without going through G4, consistent with the absence of G4 cells in the PB and SP (Fig.3d).

\section{Both known and many uncharacterized transcription factors drive neutrophil subpopulations}

We next sought to characterize transcription factor (TF) dynamics across neutrophil differentiation and maturation, since tightly regulated transcriptional programs are likely to dictate neutrophil populations (Monticelli and Natoli, 2017; Theilgaard-Monch et al., 2005). We first examined the expression of TFs known to be involved in granulopoiesis and neutrophil function (Fig.3e). Genes related to stem cell maintenance and early lineage commitment such as Gata2, Irf8, and Runx1 were highly expressed in the G0 population. Genes highly expressed in G1 included Gfi1 and Cepba, which play essential roles in neutrophil development (Avellino and Delwel, 2017; Guo et al., 2012; Hock et al., 2003; Zhang et al., 1997), strongly suggesting that specific neutrophil lineage commitment occurs during G1. Cebpa has been implicated in cessation of cell proliferation during granulopoiesis (Porse et al., 2001; Slomiany et al., 2000). However, Cebpa expression was highest in proliferating G0 and G1 cells and then drastically declined in more mature cells, with the lowest expression found in the non-dividing G3 population, making it unlikely that Cebpa directly ceases proliferation of maturating neutrophils. Similarly, Cebpe is another terminal neutrophil differentiation factor (Bjerregaard et al., 2003; Gombart et al., 2003; Gombart et al., 2001; Khanna-Gupta et al., 2007; Larsen et al., 2014; Paul et al., 2016; Verbeek et al., 2001; Yamanaka et al., 1997) implicated in promoting cell cycle arrest (Gery et al., 2004; Nakajima et al., 2006), but Cebpe transcription was highest in proliferating G2 cells (Fig.3e). Thus, the transcriptional switch ending cell proliferation stage and initiating terminal differentiation is likely to be controlled by TFs and networks other than Cebpa and Cebpe.

To assess specific global gene regulatory networks associated with neutrophil maturity, we applied SCENIC analysis (Aibar et al., 2017). Based on regulon activity, cells were projected onto a low-dimensional subspace, reproducing similar developmental trajectories to those of the Seurat projections (Fig.3f). There was high consistency between Seurat clusters and SCENIC clusters (Fig.3g). HSPCs, G0, and G1 formed an aggregate cluster, suggesting a shared "primitive" regulatory state, whereas the other four non-neutrophil populations had dramatically different TF networks. To further dissect the regulatory differences between neutrophils and other cell types, we compared regulon activities from each neutrophil group versus all non-neutrophil populations using a generalized linear model (Lambrechts et al., 2018). This identified 19 neutrophil-specific networks, including previously reported TFs such as Cebpe, Spi1, and Klf5 (Fig.3h and Table S5). Importantly, this analysis also identified four new regulons, $N f i l 3, M a x, M l x$, and Xbp1, which are closely related to the expression of neutrophil-specific genes (Fig.3i). 
We next examined the regulatory events responsible for transitioning between consecutive neutrophil differentiation stages (Fig.3j). Coarse-grained clustering revealed at least five regulon groups with distinct activation patterns, including two early-activated, one late-activated, one globally-activated, and one specifically-inactivated after G2. While many TF networks like Cebpe, Ets1, Klf5, Rad21, and E2f2 contributed to neutrophil commitment, changes in Xbp1 and $M l x$ networks were specifically associated with G0/G1 transition. Additionally, the dramatic loss of regulatory networks such as E2f1, Nelfe, and Rb1 indicated a potential functional change between G2 and G3. As well as TFs known to be essential for neutrophil differentiation and maturation, we also identified numerous novel regulons responsible for transitioning, providing a foundation for further studying the molecular basis of neutrophil maturation and heterogeneity.

\section{Bacterial infection primes neutrophils for augmented functionality without affecting overall}

\section{heterogeneity}

We next investigated how bacterial infection affected neutrophil subpopulations (Fig.S4a), including in the liver and peritoneal cavity (Fig.S4a-c). At the same sequencing depth, the gene number and total UMIs both increased in neutrophils isolated from the PB, SP, and PB of E. coli-challenged mice compared to control mice, indicating elevated transcriptional activity during bacterial infection (Fig.S4d-e). Leveraging the fact that cells in each subpopulation from unchallenged mice share common signature genes, we were able to identify every population in challenged mice using a well-accepted data integration method (Stuart et al., 2019) (Fig.4a-b) and validated independently through unsupervised dimension reduction at both transcriptome and transcription regulatory network levels (Fig.4a-b and Fig.S6a). The expression of signature molecules (Fig.4c), NADPH oxidase components (Fig.S4f), and granular proteins (Fig.S4g) remained remarkably consistent after E. coli challenge. Thus, the identity of each neutrophil population was maintained during acute bacterial infection and the signature genes still successfully determined neutrophil identity under inflammatory conditions (Fig.4c).

Despite the overall subpopulation stability during bacterial infection, infection up- and downregulated numerous genes in each neutrophil subpopulation (Fig.4d). Several cytokines involved in early leukocyte recruitment during infection such as $I l 1 b$ and Ccl6 were downregulated in neutrophils from E. coli-challenged hosts. In differential gene expression analysis (Fig.S5a and Table S6), DEGs in G0 and G1 cells were also preferentially involved in regulating immune effector processes and ROS metabolism, respectively, suggesting that immune adaptation to bacterial infection could occur as early as within early progenitor cells (Fig.4e and Fig.S5b). Under steady-state conditions, protein translation genes were highly expressed in G0 and G1 cells (Fig.1f), consistent with their highly proliferative nature. During bacterial infection, these genes and genes related to protein folding were further upregulated in G2 and G3 cells, presumably to meet the elevated protein needs of functionalized neutrophils (Fig.S5b). In relatively mature G4 and G5 neutrophils, bacterial infection triggered significant upregulation of cytokine production and secretion genes (Fig.4e and Fig.S5b). 
Interestingly, genes related to response to interferon-beta were preferentially upregulated in G5a cells, while genes related to $\mathrm{T}$ cell proliferation and activation were upregulated in G5b cells (Fig.S5b), suggesting that these two neutrophil populations play different roles in bacterial infection. Finally, in bacteria-challenged hosts, neutrophil functions related to bactericidal activities including synthesis of granular proteins (Fig.4f), NADPH oxidase complex (Fig.4g), phagocytosis, and chemotaxis (Fig.4h) were all upregulated. Thus, during bacterial infection, core neutrophil subpopulations are maintained but genes related to pathogen clearance are upregulated at each stage of neutrophil maturation to maximize host defenses.

We also examined whether bacterial infection had a universal impact on transcriptional regulatory networks across neutrophil populations. Overall, there was a coherent drift in gene regulatory network activities in each subpopulation after bacterial challenge (Fig.S6a), perhaps driven by upregulation of defense-responseassociated TF networks like Irf7 and downregulation of metabolic TF networks like Foxp1 and Ctcf(Fig.S6b). Interestingly, we also identified TF networks (e.g. Fos and Atf4) showing different responses in immature (upregulated) and mature (downregulated) populations (Fig.S6b). These networks were gradually activated from G1 to G5 under normal conditions (Fig.S6c and Table S5), and their target genes were enriched for a variety of processes including signaling, biosynthesis, and transcriptional regulation. Presumably, bacterial challenge accelerates neutrophil maturation by upregulating these networks at earlier stages and thus reallocates cellular resources to defense responses by downregulating these networks at later stages.

\section{The liver displays a distinct extramedullary granulopoiesis program during bacterial infection}

The liver is a major contributor to embryonic and fetal hematopoiesis but only a minor contributor to adult hematopoiesis (Mafra et al., 2019). In healthy mice, neutrophil numbers in the liver were so low that we could not collect enough $\mathrm{Gr}^{+}$neutrophils for scRNA-seq. Extramedullary granulopoiesis during infection generates sufficient mature neutrophils for pathogen clearance (Kim, 2010) and enable scRNA-seq. Both the percentage and total number of neutrophils increased significantly in the liver after E. coli challenge (Fig.S6d). In terms of liver neutrophil composition (Fig.S6e), the granulopoiesis trajectory in the liver was more obvious than that in the spleen, where there was almost completely overlap with PB neutrophils (Fig.4a) and the percentage of G0, G1, and G2 cells were significantly lower (Fig.S6f). Interestingly, only about half of liver neutrophils overlapped with PB or BM neutrophils (Fig.S6e), the other half (mainly G3 and G4 neutrophils) forming a distinct liver-specific population (Fig.S6e). The origin of the liver G0-G2 cells remains elusive. HSPCs are mobilized from the BM to the peripheral tissue where they may contribute to extramedullary granulopoiesis. Alternatively, they can be generated locally from more primitive cells. A previous study suggested that extramedullary hematopoiesis in the adult mouse liver is associated with specific hepatic sinusoidal endothelial cells (Cardier and Barbera-Guillem, 1997). 


\section{The ISG-related G5b neutrophil population exists in both humans and mice and expands during infection}

The percentage of G5b cells increased significantly in E. coli-challenged hosts (Fig.4b). We investigated the distribution of G5b neutrophils in the spleen using a laser scanning cytometer (LSC), co-staining spleen tissue sections with an anti-S100a8 antibody and an anti-Ifit1 antibody to identify G5b neutrophils (Fig.5a). Under normal conditions, $\mathrm{S} 100 \mathrm{a} 8^{+}$neutrophils were uniformly distributed in the red pulp, while G5b $\left(\mathrm{S} 100 \mathrm{a} 8^{+} \mathrm{Ifit}^{+}\right)$ cells were preferentially subcapsular. After E. coli challenge, overall number of neutrophils in the spleen increased significantly, as did the percentage and number of G5b (Fig.5b), which were still preferentially subcapsular, their specialized location further demonstrating the uniqueness of this subpopulation. Although multiple ISGs (e.g. Ifitm1) were upregulated in basically all neutrophil subpopulations after bacterial stimulation (Fig.5c), many ISGs such as Isg15 and Oas2 which are specifically expressed in G5b were not upregulated, suggesting that ISG-related G5b expansion was not due to bacteria-induced ISG expression. We next examined whether this G5b population was also present in human blood by scRNA-seq of sorted human PB neutrophils from a healthy donor. Unsupervised clustering revealed three major neutrophil populations, one of which was a human G5b (hG5b) population accounting for $35 \%$ of the PB neutrophils (Fig.5d-e and Table S7). Similar to mouse G5b neutrophils, hG5b only expressed a subset of ISGs (Fig.5f).

\section{Bacterial infection accelerates G1 cell division and post-mitotic maturation without altering overall neutrophil differentiation}

Neutrophil populations significantly expand during bacterial infection (Kwak et al., 2015; Manz and Boettcher, 2014). However, the neutrophil differentiation and maturation trajectory was largely maintained in E. colichallenged mice (Fig.6a). The overall stability of the neutrophil differentiation program after bacterial infection was also demonstrated by correlation of SCENIC transcription regulatory networks in control and challenged samples (Fig.6b). We next explored the mechanisms elevating neutrophil production during acute bacterial infection. While both G1 and G2 cells are proliferative, the proliferation score increased only in the G1 population (Fig.6c), as were genes related to G2/M phase progression (Tirosh et al., 2016a), while genes related to S phase progression were paradoxically reduced in G2 cells during acute infection (Fig.6d). The accelerated cell proliferation seen during emergency granulopoiesis is therefore likely to be mediated by enhanced cell proliferation in the G1 population. This was also supported by the more drastic expansion of G1 rather than G2 population during acute infection (Fig $\mathbf{4 b})$.

During bacterial infection, the G3 and G4 pool must increase to produce more mature neutrophils. Accelerated progenitor cell proliferation also suggests increased input to the post-mitotic G3 and G4 cells. Nevertheless, the proportions of G3 and G4 cells were not increased in the BM of E. coli-challenged hosts (Fig.4b), indicating that post-mitotic maturation may be accelerated in the BM. To test this hypothesis, we labeled dividing cells 
with 5-ethynyl-2'-deoxyuridine (EdU) and tracked these cells post-mitotically in the BM and PB (Fig.6e). Based on the surface expression of Ly6G and Cxcr4 in differentiating neutrophils (Eash et al., 2010; Evrard et al., 2018; Giladi et al., 2018), we separated relatively immature (Ly6 $\mathrm{G}^{\text {low }} \mathrm{Cxcr}{ }^{\text {hi }}$ ), intermediate mature $\left(\mathrm{Ly} 6 \mathrm{G}^{\text {hi }} \mathrm{Cxcr} 4^{\text {high }}\right)$, and mature $\left(\mathrm{Ly} 6 \mathrm{G}^{\text {hi }} \mathrm{Cxcr}{ }^{\text {low }}\right)$ neutrophils, and examined the emergence of EdU-labeled cells in these subpopulations at different time points. In the BM of unchallenged mice, EdU-labeled cells entered the immature neutrophil stage after $2 \mathrm{~h}$, intermediate maturation after $12 \mathrm{~h}$, and became mature neutrophils at $48 \mathrm{~h}$. The duration of each stage was about $24 \mathrm{~h}$, and EdU ${ }^{+}$cells appeared in the PB and SP after $72 \mathrm{~h}$ (Fig.6f). In E. coli-challenged hosts, the percentage of $\mathrm{Ly} 6 \mathrm{G}^{\text {hi }} \mathrm{Cxcr} 4^{\text {low }}$ cells reduced significantly, and $\mathrm{Ly} 6 \mathrm{G}^{\text {low }} \mathrm{Cxcr} 4^{\text {hi }}$ and Ly $6 \mathrm{G}^{\mathrm{hi}} \mathrm{Cxcr} 4^{\mathrm{hi}}$ cells predominated in both the BM and PB. These neutrophils mobilized to the periphery following a similar dynamic pattern but over only two rather than three days, a drastic reduction in the postmitotic neutrophil maturation period in infected hosts (Fig.6f).

\section{Bacterial infection reprograms the neutrophil population structure and dynamic transitions between subpopulations}

Bacterial infection did not alter the overall identity of each neutrophil subpopulation but instead resulted in dynamic changes in each neutrophil population. In the BM, the proportion of G1 cells increased, indicating elevated proliferation of myeloid progenitors (Fig.4b and Fig.7a). The percentage of BM G2 cells remained the same, suggesting balanced influx from G1 cells and transformation from G2 to G3 cells. In velocity analysis (Fig.7b and Fig.S7a-b), the obvious transformation from G3 to G5a cells under homeostatic conditions was suppressed during infection, and G3 cells in infected hosts predominantly differentiated to G4 cells (Fig.7b). G4 cells decreased from 38\% to $30 \%$ in the BM but significantly increased in the PB and SP (Fig.4b and Fig.7a). PB G4 cells were mainly derived from the BM G3 cells (Fig.7b). Additionally, infection significantly suppressed the G5a and G5b to G5c transition, leading to a smaller G5c population in E. coli challenged hosts (Fig.7b). To assess neutrophil heterogeneity at the site of infection, neutrophils were extracted from the peritoneal cavity (PC) (Fig.7c-d). Very few immature cells were detected, with $>97 \%$ of neutrophils being mature (G5a-c). Interestingly, although G5c cells accounted for only about 25\% of PB neutrophils before bacterial challenge and $<10 \%$ PB neutrophils after bacterial challenge, $>45 \%$ of PC neutrophils in challenged mice were G5c cells (Fig.7d), suggesting that these cells may possess higher trans-endothelial migration capability than G5a or G5b cells. Supporting this hypothesis, the number of circulating G5c cells drastically reduced from $26 \%$ to $8.2 \%$ after bacterial challenge (Fig.4b).

Infection delays neutrophil death (Luo and Loison, 2008). Paradoxically, genes related to apoptosis or necroptosis were significantly upregulated in every neutrophil subpopulation in E. coli challenged mice (Fig.7e), indicating that the delayed neutrophil death in infected hosts is mainly determined by the activation of apoptotic factors and pathways rather the level of the related proteins. 


\section{Discussion}

A complete mechanistic understanding of neutrophil differentiation and function requires detailed knowledge of the gene expression alterations driving progression from one cellular state to the next or accompanying a particular functional population. Neutrophils are transcriptionally active (Silvestre-Roig et al., 2016; Zhang et al., 2004), and many aspects of transcriptional dynamics, including epigenetic regulation, are well documented (Grassi et al., 2018; Rönnerblad et al., 2014; Schuyler et al., 2016). Nevertheless, expression profiling of bulk, heterogeneous cell populations captures average expression states that may not represent any single cell. Here we used single-cell RNA sequencing to investigate the transcriptional landscape of neutrophil maturation and fate decisions under steady-state conditions and during bacterial infection at the single-cell level. Unbiased profiling of immature and mature neutrophils in the bone marrow, peripheral blood, and spleen provides new opportunities for exploring the transcriptional landscape of neutrophil development, function, and organ distribution.

It is well accepted that neutrophils are an inhomogeneous population. However, the presence of defined neutrophil subpopulations and whether they serve specific functions are a matter of debate. A common argument is that neutrophil heterogeneity may simply represent different neutrophil developmental stages, activation states, and microenvironments. Additionally, the specific function of circulating neutrophils lies on a continuum due to their rapid aging, the effect of the gastrointestinal microbiome, and accrued cellular damage as neutrophils traverse narrow capillaries. Such phenotypic differences should not be regarded as neutrophil subpopulations if all neutrophils generated in the BM or PB can exhibit those features. Our analysis revealed five BM neutrophil subpopulations (including the GMP population) during development and maturation including three dividing subsets and two post-mitotic maturing subsets. The peripheral blood contained three distinct neutrophil subsets defined by distinct molecular signatures. Their identity was stable and largely maintained during bacterial infection.

The discrete and definable ISG-expressing G5b neutrophil subpopulation was present in both humans and mice, expanded during bacterial infection, and was independent of neutrophil activation. These cells highly expressed a set of ISGs and increased in number in E. coli-challenged hosts. Although G5b neutrophils were more similar to G5a than G5c neutrophils, the majority of G5b neutrophils directly developed from BM G4 cells (Fig.7f). In the spleens of $E$. coli-stimulated hosts, $\mathrm{Gr}^{+}$neutrophils were uniformly distributed in the red pup, while G5b cells preferentially localized to the subcapsular region, further demonstrating that the G5b neutrophil subpopulation is unique and suggesting that this subpopulation may be pre-programmed with a different host defense function. Interferon and interferon-related pathways are implicated in both viral and non-viral infections and play a critical role in host defenses (Schneider et al., 2014). ISG-related G5b neutrophils may be primed to combat invading pathogens even before infection occurs. Thus, the G5b neutrophil subset appears to be a 
functionally different population with distinct molecular signatures that maintain their features throughout the short neutrophil lifespan.

This study generated the first comprehensive single-cell RNA-seq map of neutrophils under physiological conditions and during bacterial infection. A number of neutrophil subpopulations have previously been identified through functional and phenotypic associations in various models, including but are not limited to human CD177 (Hu et al., 2009; Silvestre-Roig et al., 2016; Wu et al., 2016; Zhou et al., 2018), olfactomedin$4^{+}$(OLFM4) (Clemmensen et al., 2012), TCR-expressing (Puellmann et al., 2006), CD49d $\mathrm{d}^{+}$EEGR ${ }^{\text {high }} \mathrm{CXCR} 4{ }^{\text {high }}$ angiogenic (Christoffersson et al., 2012; Massena et al., 2015), CD63 ${ }^{+}$ (Tirouvanziam et al., 2008), IL-13 (Chen et al., 2014), CD49+ (Cheung et al., 2010; Tsuda et al., 2004), IL-17producing (Taylor et al., 2014), CD62 L ${ }^{\mathrm{dim}} / \mathrm{CD} 16^{\text {bright }}$ and CD62L $\mathrm{L}^{\text {bright} / C D 16 ~}{ }^{\mathrm{dim}}$ (Pillay et al., 2012), immunosuppressive CD11 $\mathrm{c}^{\text {bright }} \mathrm{CD} 62 \mathrm{~L}^{\text {dim }} \mathrm{CD} 11 \mathrm{~b}^{\text {bright }} \mathrm{CD} 16^{\text {bright }}$ (Pillay et al., 2012), CD16 ${ }^{\text {dim }}$ banded (Leliefeld et al., 2018), CD62L ${ }^{\text {dim }}$ (Tak et al., 2017), mature CD10 ${ }^{+}$and immature CD10- (Marini et al., 2017), and tumorelicited immature c-Kit ${ }^{+}$(Rice et al., 2018) neutrophils. Additionally, Buckley et al. identified a subset of human CD54 (ICAM-1) ${ }^{\text {high } C X C R 1 ~}{ }^{\text {low }} V_{E G F R}{ }^{\text {high }}$ neutrophils representing cells migrating through an endothelial monolayer and then re-emerging by reverse transmigration (RT) (Buckley et al., 2006). More recently, Deniset et al revealed that splenic Ly $6 \mathrm{G}^{\text {high }}$ mature and Ly6 $\mathrm{G}^{\text {int }}$ immature neutrophils contribute to eradication of $S$. pneumoniae. Resident neutrophil progenitors $\left(\mathrm{CD} 11 \mathrm{~b}^{+} \mathrm{Ly} 6 \mathrm{G}^{\mathrm{int}} \mathrm{c}-\mathrm{Kit}^{+}\right)$in the spleen undergo emergency proliferation and mobilization from their splenic niche after pneumococcal stimulation to increase the effector mature neutrophil pool (Deniset et al., 2017). The molecular signatures of most of the aforementioned neutrophil subpopulations are unknown. Whether any of these neutrophil subpopulations overlap with or are derived from a particular neutrophil subset identified in current study will need to be determined.

Neutrophils are now known to be important in cancer. Two tumor-associated neutrophil (TAN) subpopulations are present in tumors: pro-inflammatory antitumorigenic N1 neutrophils and pro-tumorigenic N2 neutrophils (Fridlender et al., 2009; Giese et al., 2019; Sionov et al., 2015). N1 TANs showed a more immunostimulatory mRNA profile than N2 neutrophils, expressing higher levels of TNF- $\alpha$, CCL3, iNOS, and ICAM-1. In contrast, N2 TANs express higher levels of CCL2, CCL5, CCL17, VEGF, and arginase, an important immunosuppressor of the adaptive immune system (Fridlender et al., 2009; Mishalian et al., 2013; Shaul et al., 2016). In a mouse melanoma model, IFN- $\beta$ deficiency expands the number of TANs expressing elevated levels of genes encoding the proangiogenic factors VEGF and MMP9, CXCR4, and the transcription factors c-myc and STAT3, thereby augmenting tumor angiogenesis and growth (Jablonska et al., 2010). Sagiv et al. identified three distinct circulating neutrophil populations in cancer: high-density mature small anti-tumor neutrophils, low-density mature large pro-tumor neutrophils, and low-density immature large pro-tumor granulocyte-myeloid-derived suppressor cells (G-MDSC). MET expression, induced by inflammatory stimuli, is required for the recruitment 
of anti-tumor neutrophils (Finisguerra et al., 2015). Low-density neutrophils (LDN) were defined due to their appearance in the low-density layer of a Ficoll gradient (Carmona-Rivera and Kaplan, 2013; Garcia-Romo et al., 2011; Hacbarth and Kajdacsy-Balla, 1986). These cells may be responsible for sustaining chronic inflammation and have elevated capability to form NETs (Kanamaru et al., 2018; Khandpur et al., 2013). LDNs have a gene expression signature associated with a reduced inflammatory state (Sagiv et al., 2015). G-MDSC represents a group of immature neutrophils with immunosuppressive functions (Tcyganov et al., 2018; Veglia et

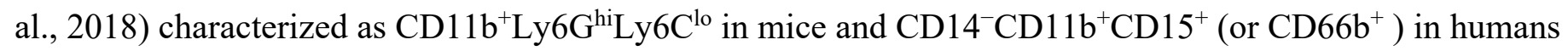
(Bronte et al., 2016). Condamine et al. identified lectin-type oxidized LDL receptor 1 (LOX-1) as a specific marker of human G-MDSCs associated with endoplasmic reticulum stress and lipid metabolism in cancer patients (Condamine et al., 2016). Comparative transcriptomic analyses of TANs, G-MDSCs, and naïve neutrophils suggested that these three subpopulations are distinct, with an unexpected close proximity of GMDSCs and naïve neutrophils compared with TANs (Fridlender et al., 2012). Structural genes and genes related to cytotoxicity were significantly downregulated in TANs. In contrast, many immune-related genes and pathways, including genes related to the antigen-presenting complex (e.g., MHC-II complex genes) and cytokines (e.g., TNF- $\alpha$, IL-1- $\alpha / \beta$ ), were upregulated in G-MDSCs and further upregulated in TANs. We examined the expression of genes related to each tumor-related neutrophil subpopulation but could not correlate any of these neutrophil subsets with any of our novel subpopulations, suggesting that these specialized neutrophils are reprogrammed and acquire unique molecular signatures in the tumor-bearing host. Alternatively, these functionally defined neutrophil subsets, whose identities often rely on expression of a few surface markers, may still be heterogenous, leading to difficulty in recognizing the involved neutrophil populations. Interestingly, a group of ISG-expressing tumor-infiltrating neutrophils was recently identified in human and mouse lung cancers (Zilionis et al., 2019). Nevertheless, the transcriptome of this neutrophil population is different to that of G5b neutrophils, again indicating significant neutrophil reprogramming in the tumor microenvironment.

Using mass cytometry (CyTOF) and cell cycle-based analysis, Evrard et al. identified three neutrophil subsets within the bone marrow: committed c-Kit ${ }^{\text {low/int }}$ proliferative neutrophil precursors expressing primary and secondary granule proteins (preNeu), $\mathrm{CXCR} 2^{\text {low }}$ non-proliferating immature neutrophils highly expressing secondary granule proteins, and CXCR2 ${ }^{\text {high }}$ mature neutrophils highly expressing gelatinase granule proteins (Evrard et al., 2018). Based on these features, these three populations are likely correlated with G1/G2, G3, and G4 neutrophils identified in our study based on RNA-seq data (Graphical Abstract). More recently, a proliferative unipotent neutrophil precursor that suppresses $\mathrm{T}$ cell activation and promotes tumor growth was identified in the mouse bone marrow that generates neutrophils after intra-bone marrow adoptive transfer. ScRNA-seq analysis of these cells further revealed two populations: an early-stage ckit ${ }^{+} \mathrm{Gfi} 1{ }^{\text {low }} \mathrm{Cebpa}^{\text {hi }} \mathrm{Ly} 6 \mathrm{G}^{\text {low }}$ progenitor with stem cell morphology and a late-stage ckit ${ }^{+} \mathrm{Gfi}^{\mathrm{hi}} \mathrm{Cebpa}^{\text {low }} \mathrm{Ly} 6 \mathrm{G}^{+}$precursor with morphological 
features similar to transient neutrophil precursors (Zhu et al., 2018). Further analysis suggested that the latestage progenitors were mostly similar to the preNeu population identified by Evrard et al. (Zhu et al., 2018) (Graphical Abstract). Notably, in an earlier study, Kim et al. also defined a population of proliferative late-

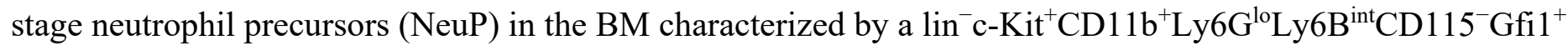
signature (Kim et al., 2017) that should be located in the G1/G2 population. It was later found that this NeuP population was highly heterogeneous and contained other myeloid progenitors (G0 cells) (Zhu et al., 2018). It is commonly believed that neutrophils mobilize from the BM to the PB only when they become fully mature. RNA velocity analysis revealed unexpected complexity of neutrophil mobilization from the BM to PB. Under homeostatic conditions, a significant number of G3 neutrophils were mobilized to the PB or tissue without going through the most mature G4 cells. Collectively, these results demonstrated that immature PB neutrophils in healthy hosts are mainly derived from more primitive early stage post-mitotic G3 cells, which were more mobile than the more mature G4 cells. Thus, full maturation may not be a pre-requisite for neutrophil mobilization. Early-stage post-mitotic G3 cells can be mobilized from the BM to the periphery via a maturationindependent mechanism to complete their development in the PB and/or SP (Fig.7f). Interestingly, the transformation from G3 to G5a cells was suppressed during infection and G3 cells in infected hosts predominantly differentiated to G4 cells.

Infection, long-term inflammation, and cancer can all reprogram granulopoiesis to produce neutrophils with different gene expression patterns. Indeed, we observed global changes in the neutrophil transcriptome in response to a bacterial stimulus. Nevertheless, the core identity of each neutrophil population and the overall neutrophil differentiation program remained the same during bacterial infection. The infection-triggered increase in neutrophils was mainly due to accelerated cell division in the more primitive G1 population rather than the G2 population, which was responsible for the majority of cell proliferation during homeostasis. One explanation for such a mechanism is that, under steady-state conditions, proliferation of G2 cells is already relatively high, so accelerating division of the more primitive G1 cells would provide a more efficient way to expand the neutrophil population. Infection also significantly shortened the period of post-mitotic maturation, leading to faster turnover of maturing neutrophils in the BM. Taken together, bacterial infection reprogrammed the structure of the neutrophil population and the dynamic transition between each subpopulation but did not produce distinct, functionally different neutrophil subpopulations in infected hosts.

\section{ACKNOWLEDGEMENTS}

Cell sorting was performed at the HSCI/DRC Flow Core (NIH P30DK036836). H. Luo is supported by National Institutes of Health grants (1 R01 AI142642, 1 R01 AI145274, 1 R01 AI141386, R01HL092020, and P01 HL095489) and a grant from FAMRI (CIA 123008). Q.S. and C.L. were supported by Natural Science 
Foundation of China (31871266), Chinese National Key Projects of Research and Development (2016YFA0100103), and NSFC Key Research Grant 71532001. F. Ma is supported by the grants from National Basic Research Program of China (2015CB964903), Chinese Academy of Medical Sciences (CAMS) Innovation Fund for Medical Sciences (2017-I2M-1-015, 2016-I2M-1-017), the Non-profit Central Research Institute Fund of Chinese Academy of Medical Sciences (2018RC31002, 2018PT32034, 2017PT31033), National Natural Sciences Foundation of China (31271484 and 31471116), Natural Science Foundation of Tianjin City (18JCYBJC25700).

\section{AUTHOR CONTRI BUTIONS}

Conceptualization, H.R.L., C.L., and F.M.; Methodology, H.R.L., L.S., and C.L.; Investigation, X.X., Q.S., P.W., X.Z., J.S., R.G., Q.R., S.Z., H.Y., S.P. and H.K.; Formal Analysis, X.X., Q.S., P.W., X.Z., J.S., R.G.,

Q.R., S.Z., H.Y., S.P. and H.K.; Writing - Original Draft, H.R.L., X.X., J.S., and Q.S.; Writing -Review \& Editing, H.R.L., C.L., X.X., J.S., Q.S., and F.M.; Funding Acquisition, T.C., C.L., F.M., L.S. and H.R.L.; Resources, H.R.L., C.L., T.C., Y.X., and L.S.; Supervision, H.R.L., Y.X., C.L., and F.M.; Project Administration, H.R.L., C.L., Y.X., T.C., and F.M.

\section{DECLARATION OF INTERESTS}

The authors declare no competing financial interests. 


\section{REFERENCES}

Adlung, L., and Amit, I. (2018). From the Human Cell Atlas to dynamic immune maps in human disease. Nat Rev Immunol 18, 597-598.

Adrover, J.M., Del Fresno, C., Crainiciuc, G., Cuartero, M.I., Casanova-Acebes, M., Weiss, L.A., HuergaEncabo, H., Silvestre-Roig, C., Rossaint, J., Cossio, I., et al. (2019). A Neutrophil Timer Coordinates Immune Defense and Vascular Protection. Immunity 50, 390-402.e310.

Adrover, J.M., Nicolas-Avila, J.A., and Hidalgo, A. (2016). Aging: A Temporal Dimension for Neutrophils. Trends Immunol 37, 334-345.

Aibar, S., González-Blas, C.B., Moerman, T., Imrichova, H., Hulselmans, G., Rambow, F., Marine, J.-C., Geurts, P., Aerts, J., and van den Oord, J. (2017). SCENIC: single-cell regulatory network inference and clustering. Nature methods 14,1083\%@1548-7105.

Avellino, R., and Delwel, R. (2017). Expression and regulation of C/EBPalpha in normal myelopoiesis and in malignant transformation. Blood 129, 2083-2091.

Babior, B.M., Lambeth, J.D., and Nauseef, W. (2002). The neutrophil NADPH oxidase. Arch Biochem Biophys $397,342-344$.

Baggiolini, M. (2001). Chemokines in pathology and medicine. J Intern Med 250, 91-104.

Becht, E., McInnes, L., Healy, J., Dutertre, C.-A., Kwok, I.W.H., Ng, L.G., Ginhoux, F., and Newell, E.W. (2018a). Dimensionality reduction for visualizing single-cell data using UMAP. Nature biotechnology 37, 38.

Becht, E., McInnes, L., Healy, J., Dutertre, C.A., Kwok, I.W.H., Ng, L.G., Ginhoux, F., and Newell, E.W. (2018b). Dimensionality reduction for visualizing single-cell data using UMAP. Nat Biotechnol.

Benaglia, T., Chauveau, D., Hunter, D., and Young, D. (2009). mixtools: An R package for analyzing finite mixture models.

Bjerregaard, M.D., Jurlander, J., Klausen, P., Borregaard, N., and Cowland, J.B. (2003). The in vivo profile of transcription factors during neutrophil differentiation in human bone marrow. Blood 101, 4322-4332.

Bolger, A.M., Lohse, M., and Usadel, B. (2014). Trimmomatic: a flexible trimmer for Illumina sequence data. Bioinformatics 30, 2114-2120.

Borregaard, N., and Cowland, J.B. (1997). Granules of the human neutrophilic polymorphonuclear leukocyte. Blood 89, 3503-3521.

Borregaard, N., and Herlin, T. (1982). Energy Metabolism of Human Neutrophils during Phagocytosis. The Journal of Clinical Investigation 70, 550-557. 
Borregaard, N., Sorensen, O.E., and Theilgaard-Monch, K. (2007). Neutrophil granules: a library of innate immunity proteins. Trends Immunol 28, 340-345.

Bronte, V., Brandau, S., Chen, S.H., Colombo, M.P., Frey, A.B., Greten, T.F., Mandruzzato, S., Murray, P.J., Ochoa, A., Ostrand-Rosenberg, S., et al. (2016). Recommendations for myeloid-derived suppressor cell nomenclature and characterization standards. Nature communications 7, 12150.

Broxmeyer, H.E. (2008). Chemokines in hematopoiesis. Curr Opin Hematol 15, 49-58.

Buckley, C.D., Ross, E.A., McGettrick, H.M., Osborne, C.E., Haworth, O., Schmutz, C., Stone, P.C., Salmon, M., Matharu, N.M., Vohra, R.K., et al. (2006). Identification of a phenotypically and functionally distinct population of long-lived neutrophils in a model of reverse endothelial migration. J Leukoc Biol 79, 303-311.

Bunting, M., Harris, E.S., McIntyre, T.M., Prescott, S.M., and Zimmerman, G.A. (2002). Leukocyte adhesion deficiency syndromes: adhesion and tethering defects involving beta 2 integrins and selectin ligands. Curr Opin Hematol 9, 30-35.

Burg, N.D., and Pillinger, M.H. (2001). The neutrophil: function and regulation in innate and humoral immunity. Clin Immunol 99, 7-17.

Butler, A., Hoffman, P., Smibert, P., Papalexi, E., and Satija, R. (2018). Integrating single-cell transcriptomic data across different conditions, technologies, and species. Nat Biotechnol.

Cardier, J.E., and Barbera-Guillem, E. (1997). Extramedullary hematopoiesis in the adult mouse liver is associated with specific hepatic sinusoidal endothelial cells. Hepatology (Baltimore, Md) 26, 165-175.

Carmona-Rivera, C., and Kaplan, M.J. (2013). Low-density granulocytes: a distinct class of neutrophils in systemic autoimmunity. Semin Immunopathol 35, 455-463.

Casanova-Acebes, M., Pitaval, C., Weiss, L.A., Nombela-Arrieta, C., Chèvre, R., A-González, N., Kunisaki, Y., Zhang, D., van Rooijen, N., Silberstein, L.E., et al. (2013). Rhythmic modulation of the hematopoietic niche through neutrophil clearance. Cell 153, 1025-1035.

Castanheira, F.V.S., and Kubes, P. (2019). Neutrophils and NETs in modulating acute and chronic inflammation. Blood 133, 2178-2185.

Chen, F., Wu, W., Millman, A., Craft, J.F., Chen, E., Patel, N., Boucher, J.L., Urban, J.F., Jr., and Kim, C.C. (2014). Neutrophils prime a long-lived effector macrophage phenotype that mediates accelerated helminth expulsion. $15,938-946$.

Cheraghali, A.M., Kumar, R., Knaus, E.E., and Wiebe, L.I. (1995). Pharmacokinetics and bioavailability of 5ethyl-2'-deoxyuridine and its novel (5R,6R)-5-bromo-6-ethoxy-5,6-dihydro prodrugs in mice. Drug metabolism and disposition: the biological fate of chemicals 23, 223-226. 
Cheung, D.S., Ehlenbach, S.J., Kitchens, R.T., Riley, D.A., Thomas, L.L., Holtzman, M.J., and Grayson, M.H. (2010). Cutting edge: CD49d+ neutrophils induce FcepsilonRI expression on lung dendritic cells in a mouse model of postviral asthma. J Immunol 185, 4983-4987.

Christoffersson, G., Vagesjo, E., Vandooren, J., Liden, M., Massena, S., Reinert, R.B., Brissova, M., Powers, A.C., Opdenakker, G., and Phillipson, M. (2012). VEGF-A recruits a proangiogenic MMP-9-delivering neutrophil subset that induces angiogenesis in transplanted hypoxic tissue. Blood 120, 4653-4662.

Clemmensen, S.N., Bohr, C.T., Rorvig, S., Glenthoj, A., Mora-Jensen, H., Cramer, E.P., Jacobsen, L.C., Larsen, M.T., Cowland, J.B., Tanassi, J.T., et al. (2012). Olfactomedin 4 defines a subset of human neutrophils. J Leukoc Biol 91, 495-500.

Condamine, T., Dominguez, G.A., Youn, J.I., Kossenkov, A.V., Mony, S., Alicea-Torres, K., Tcyganov, E., Hashimoto, A., Nefedova, Y., Lin, C., et al. (2016). Lectin-type oxidized LDL receptor-1 distinguishes population of human polymorphonuclear myeloid-derived suppressor cells in cancer patients. Science immunology 1.

Consortium, G.O. (2018). The gene ontology resource: 20 years and still GOing strong. Nucleic acids research 47, D330-D338.

Cossio, I., Lucas, D., and Hidalgo, A. (2019). Neutrophils as regulators of the hematopoietic niche. Blood 133, $2140-2148$.

Cowland, J.B., and Borregaard, N. (2016a). Granulopoiesis and granules of human neutrophils. Immunol Rev 273, 11-28.

Cowland, J.B., and Borregaard, N. (2016b). Granulopoiesis and granules of human neutrophils. Immunological reviews 273, 11-28.

Dahlgren, C., and Karlsson, A. (1999). Respiratory burst in human neutrophils. J Immunol Methods 232, 3-14.

Darrah, E., and Andrade, F. (2012). NETs: the missing link between cell death and systemic autoimmune diseases? Frontiers in immunology 3, 428.

Davis, C., Fischer, J., Ley, K., and Sarembock, I.J. (2003). The role of inflammation in vascular injury and repair. Journal of thrombosis and haemostasis : JTH 1, 1699-1709.

Deniset, J.F., Surewaard, B.G., Lee, W.-Y., and Kubes, P. (2017). Splenic Ly6G ${ }^{\text {high }}$ mature and Ly6Gi ${ }^{\text {nt }}$ immature neutrophils contribute to eradication of S. pneumoniae. The Journal of Experimental Medicine 214, $1333-1350$.

Dinauer, M.C. (2019). Inflammatory consequences of inherited disorders affecting neutrophil function. Blood 133, 2130-2139. 
Dobin, A., Davis, C.A., Schlesinger, F., Drenkow, J., Zaleski, C., Jha, S., Batut, P., Chaisson, M., and Gingeras, T.R. (2013). STAR: ultrafast universal RNA-seq aligner. Bioinformatics 29, 15-21.

Doerschuk, C.M. (2000). Leukocyte trafficking in alveoli and airway passages. Respir Res 1, 136-140.

Doerschuk, C.M., Markos, J., Coxson, H.O., English, D., and Hogg, J.C. (1994). Quantitation of neutrophil migration in acute bacterial pneumonia in rabbits. Journal of applied physiology (Bethesda, Md : 1985) 77, 2593-2599.

Eash, K.J., Greenbaum, A.M., Gopalan, P.K., and Link, D.C. (2010). CXCR2 and CXCR4 antagonistically regulate neutrophil trafficking from murine bone marrow. J Clin Invest 120, 2423-2431.

Eash, K.J., Means, J.M., White, D.W., and Link, D.C. (2009). CXCR4 is a key regulator of neutrophil release from the bone marrow under basal and stress granulopoiesis conditions. Blood 113, 4711-4719.

Evrard, M., Kwok, I.W.H., Chong, S.Z., Teng, K.W.W., Becht, E., Chen, J., Sieow, J.L., Penny, H.L., Ching, G.C., Devi, S., et al. (2018). Developmental Analysis of Bone Marrow Neutrophils Reveals Populations Specialized in Expansion, Trafficking, and Effector Functions. Immunity 48, 364-379.e368.

Ewels, P., Magnusson, M., Lundin, S., and Kaller, M. (2016). MultiQC: summarize analysis results for multiple tools and samples in a single report. Bioinformatics 32, 3047-3048.

Finisguerra, V., Di Conza, G., Di Matteo, M., Serneels, J., Costa, S., Thompson, A.A., Wauters, E., Walmsley, S., Prenen, H., Granot, Z., et al. (2015). MET is required for the recruitment of anti-tumoural neutrophils. Nature 522, 349-353.

Fodil, N., Langlais, D., and Gros, P. (2016). Primary Immunodeficiencies and Inflammatory Disease: A Growing Genetic Intersection. Trends Immunol 37, 126-140.

Fridlender, Z.G., Sun, J., Kim, S., Kapoor, V., Cheng, G., Ling, L., Worthen, G.S., and Albelda, S.M. (2009). Polarization of tumor-associated neutrophil phenotype by TGF-beta: "N1" versus "N2" TAN. Cancer Cell 16, 183-194.

Fridlender, Z.G., Sun, J., Mishalian, I., Singhal, S., Cheng, G., Kapoor, V., Horng, W., Fridlender, G., Bayuh, R., Worthen, G.S., et al. (2012). Transcriptomic analysis comparing tumor-associated neutrophils with granulocytic myeloid-derived suppressor cells and normal neutrophils. PLoS One 7, e31524.

Furze, R.C., and Rankin, S.M. (2008). Neutrophil mobilization and clearance in the bone marrow. Immunology $125,281-288$.

Garcia-Romo, G.S., Caielli, S., Vega, B., Connolly, J., Allantaz, F., Xu, Z., Punaro, M., Baisch, J., Guiducci, C., Coffman, R.L., et al. (2011). Netting neutrophils are major inducers of type I IFN production in pediatric systemic lupus erythematosus. Sci Transl Med 3, 73ra20. 
Gery, S., Gombart, A.F., Fung, Y.K., and Koeffler, H.P. (2004). C/EBPepsilon interacts with retinoblastoma and E2F1 during granulopoiesis. Blood 103, 828-835.

Giese, M.A., Hind, L.E., and Huttenlocher, A. (2019). Neutrophil plasticity in the tumor microenvironment. Blood 133, 2159-2167.

Giladi, A., Paul, F., Herzog, Y., Lubling, Y., Weiner, A., Yofe, I., Jaitin, D., Cabezas-Wallscheid, N., Dress, R., Ginhoux, F., et al. (2018). Single-cell characterization of haematopoietic progenitors and their trajectories in homeostasis and perturbed haematopoiesis. Nat Cell Biol 20, 836-846.

Gombart, A.F., Kwok, S.H., Anderson, K.L., Yamaguchi, Y., Torbett, B.E., and Koeffler, H.P. (2003). Regulation of neutrophil and eosinophil secondary granule gene expression by transcription factors C/EBP epsilon and PU.1. Blood 101, 3265-3273.

Gombart, A.F., Shiohara, M., Kwok, S.H., Agematsu, K., Komiyama, A., and Koeffler, H.P. (2001).

Neutrophil-specific granule deficiency: homozygous recessive inheritance of a frameshift mutation in the gene encoding transcription factor CCAAT/enhancer binding protein--epsilon. Blood 97, 2561-2567.

Grassi, L., Pourfarzad, F., Ullrich, S., Merkel, A., Were, F., Carrillo-de-Santa-Pau, E., Yi, G., Hiemstra, I.H., Tool, A.T.J., Mul, E., et al. (2018). Dynamics of Transcription Regulation in Human Bone Marrow Myeloid Differentiation to Mature Blood Neutrophils. Cell reports 24, 2784-2794.

Gu, Z., Eils, R., and Schlesner, M. (2016). Complex heatmaps reveal patterns and correlations in multidimensional genomic data. Bioinformatics 32,2847-2849\%@1460-2059.

Guo, H., Ma, O., Speck, N.A., and Friedman, A.D. (2012). Runx1 deletion or dominant inhibition reduces Cebpa transcription via conserved promoter and distal enhancer sites to favor monopoiesis over granulopoiesis. Blood 119, 4408-4418.

Hacbarth, E., and Kajdacsy-Balla, A. (1986). Low density neutrophils in patients with systemic lupus erythematosus, rheumatoid arthritis, and acute rheumatic fever. Arthritis Rheum 29, 1334-1342.

Harnett, M.M. (2007). Laser scanning cytometry: understanding the immune system in situ. Nat Rev Immunol 7, 897-904.

Henderson, L.M., and Chappel, J.B. (1996). NADPH oxidase of neutrophils. Biochim Biophys Acta 1273, 87107.

Heyworth, P.G., Cross, A.R., and Curnutte, J.T. (2003). Chronic granulomatous disease. Curr Opin Immunol $15,578-584$. 
Hock, H., Hamblen, M.J., Rooke, H.M., Traver, D., Bronson, R.T., Cameron, S., and Orkin, S.H. (2003). Intrinsic requirement for zinc finger transcription factor Gfi-1 in neutrophil differentiation. Immunity 18, 109120.

Hu, N., Westra, J., Huitema, M.G., Bijl, M., Brouwer, E., Stegeman, C.A., Heeringa, P., Limburg, P.C., and Kallenberg, C.G. (2009). Coexpression of CD177 and membrane proteinase 3 on neutrophils in antineutrophil cytoplasmic autoantibody-associated systemic vasculitis: anti-proteinase 3-mediated neutrophil activation is independent of the role of CD177-expressing neutrophils. Arthritis Rheum 60, 1548-1557.

Jablonska, J., Leschner, S., Westphal, K., Lienenklaus, S., and Weiss, S. (2010). Neutrophils responsive to endogenous IFN-beta regulate tumor angiogenesis and growth in a mouse tumor model. J Clin Invest 120, 1151-1164.

Jaiswal, S., Jamieson, C.H., Pang, W.W., Park, C.Y., Chao, M.P., Majeti, R., Traver, D., van Rooijen, N., and Weissman, I.L. (2009). CD47 is upregulated on circulating hematopoietic stem cells and leukemia cells to avoid phagocytosis. Cell 138, 271-285.

Kanamaru, R., Ohzawa, H., Miyato, H., Matsumoto, S., Haruta, H., Kurashina, K., Saito, S., Hosoya, Y., Yamaguchi, H., Yamashita, H., et al. (2018). Low density neutrophils (LDN) in postoperative abdominal cavity assist the peritoneal recurrence through the production of neutrophil extracellular traps (NETs). Scientific reports 8,632 .

Karamitros, D., Stoilova, B., Aboukhalil, Z., Hamey, F., Reinisch, A., Samitsch, M., Quek, L., Otto, G., Repapi, E., Doondeea, J., et al. (2018). Single-cell analysis reveals the continuum of human lympho-myeloid progenitor cells. Nature Immunology 19, 85-97.

Katharine M. Mullen, I.H.M.v.S. (2012). nnls: The Lawson-Hanson algorithm for non-negative least squares (NNLS).

Khandpur, R., Carmona-Rivera, C., Vivekanandan-Giri, A., Gizinski, A., Yalavarthi, S., Knight, J.S., Friday, S., Li, S., Patel, R.M., Subramanian, V., et al. (2013). NETs are a source of citrullinated autoantigens and stimulate inflammatory responses in rheumatoid arthritis. Sci Transl Med 5, 178 ra140.

Khanna-Gupta, A., Sun, H., Zibello, T., Lee, H.M., Dahl, R., Boxer, L.A., and Berliner, N. (2007). Growth factor independence-1 (Gfi-1) plays a role in mediating specific granule deficiency (SGD) in a patient lacking a gene-inactivating mutation in the C/EBPepsilon gene. Blood 109, 4181-4190.

Kim, C.H. (2010). Homeostatic and pathogenic extramedullary hematopoiesis. Journal of blood medicine 1, 1319. 
Kim, M.-H., Yang, D., Kim, M., Kim, S.-Y., Kim, D., and Kang, S.-J. (2017). A late-lineage murine neutrophil precursor population exhibits dynamic changes during demand-adapted granulopoiesis. Scientific reports 7 , 39804.

Kjeldsen, L., Bainton, D.F., Sengelov, H., and Borregaard, N. (1993). Structural and functional heterogeneity among peroxidase-negative granules in human neutrophils: identification of a distinct gelatinase-containing granule subset by combined immunocytochemistry and subcellular fractionation. Blood 82, 3183-3191.

Kjeldsen, L., Bainton, D.F., Sengelov, H., and Borregaard, N. (1994). Identification of neutrophil gelatinaseassociated lipocalin as a novel matrix protein of specific granules in human neutrophils. Blood 83, 799-807.

Klausen, P., Bjerregaard, M.D., Borregaard, N., and Cowland, J.B. (2004). End-stage differentiation of neutrophil granulocytes in vivo is accompanied by up-regulation of p27kip1 and down-regulation of CDK2, CDK4, and CDK6. J Leukoc Biol 75, 569-578.

Kolaczkowska, E. (2016). The older the faster: aged neutrophils in inflammation. Blood 128, 2280-2282.

Kolaczkowska, E., Jenne, C.N., Surewaard, B.G., Thanabalasuriar, A., Lee, W.Y., Sanz, M.J., Mowen, K., Opdenakker, G., and Kubes, P. (2015). Molecular mechanisms of NET formation and degradation revealed by intravital imaging in the liver vasculature. Nature communications 6, 6673.

Kowalczyk, M.S., Tirosh, I., Heckl, D., Rao, T.N., Dixit, A., Haas, B.J., Schneider, R.K., Wagers, A.J., Ebert, B.L., and Regev, A. (2015). Single-cell RNA-seq reveals changes in cell cycle and differentiation programs upon aging of hematopoietic stem cells. Genome research 25, 1860-1872.

Kruger, P., Saffarzadeh, M., Weber, A.N., Rieber, N., Radsak, M., von Bernuth, H., Benarafa, C., Roos, D., Skokowa, J., and Hartl, D. (2015). Neutrophils: Between host defence, immune modulation, and tissue injury. PLoS Pathog 11, e1004651.

Kwak, H.J., Liu, P., Bajrami, B., Xu, Y., Park, S.Y., Nombela-Arrieta, C., Mondal, S., Sun, Y., Zhu, H., Chai, L., et al. (2015). Myeloid cell-derived reactive oxygen species externally regulate the proliferation of myeloid progenitors in emergency granulopoiesis. Immunity 42, 159-171.

La Manno, G., Soldatov, R., Zeisel, A., Braun, E., Hochgerner, H., Petukhov, V., Lidschreiber, K., Kastriti, M.E., Lonnerberg, P., Furlan, A., et al. (2018a). RNA velocity of single cells. Nature 560, 494-498.

La Manno, G., Soldatov, R., Zeisel, A., Braun, E., Hochgerner, H., Petukhov, V., Lidschreiber, K., Kastriti, M.E., Lönnerberg, P., Furlan, A., et al. (2018b). RNA velocity of single cells. Nature 560, 494-498.

Lambrechts, D., Wauters, E., Boeckx, B., Aibar, S., Nittner, D., Burton, O., Bassez, A., Decaluwé, H., Pircher, A., and Van den Eynde, K. (2018). Phenotype molding of stromal cells in the lung tumor microenvironment. Nature medicine 24,1277\%@1546-1170X. 
Larsen, M.T., Hager, M., Glenthoj, A., Asmar, F., Clemmensen, S.N., Mora-Jensen, H., Borregaard, N., and Cowland, J.B. (2014). miRNA-130a regulates C/EBP-epsilon expression during granulopoiesis. Blood 123, 1079-1089.

Lawrence, S.M., Corriden, R., and Nizet, V. (2018). The Ontogeny of a Neutrophil: Mechanisms of Granulopoiesis and Homeostasis. Microbiology and molecular biology reviews : MMBR 82.

Lee, W.L., Harrison, R.E., and Grinstein, S. (2003). Phagocytosis by neutrophils. Microbes Infect 5, $1299-1306$. Leliefeld, P.H.C., Pillay, J., Vrisekoop, N., Heeres, M., Tak, T., Kox, M., Rooijakkers, S.H.M., Kuijpers, T.W., Pickkers, P., Leenen, L.P.H., et al. (2018). Differential antibacterial control by neutrophil subsets. Blood advances 2, 1344-1355.

Ley, K., Hoffman, H.M., Kubes, P., Cassatella, M.A., Zychlinsky, A., Hedrick, C.C., and Catz, S.D. (2018). Neutrophils: New insights and open questions. Science immunology 3.

Luo, H.R., and Loison, F. (2008). Constitutive neutrophil apoptosis: mechanisms and regulation. Am J Hematol 83, 288-295.

Mafra, K., Nakagaki, B.N., Castro Oliveira, H.M., Rezende, R.M., Antunes, M.M., and Menezes, G.B. (2019). The liver as a nursery for leukocytes. J Leukoc Biol.

Manz, M.G., and Boettcher, S. (2014). Emergency granulopoiesis. Nat Rev Immunol 14, 302-314.

Marini, O., Costa, S., Bevilacqua, D., Calzetti, F., Tamassia, N., Spina, C., De Sabata, D., Tinazzi, E., Lunardi, C., Scupoli, M.T., et al. (2017). Mature CD10(+) and immature CD10(-) neutrophils present in G-CSF-treated donors display opposite effects on T cells. Blood 129, 1343-1356.

Martin, C., Burdon, P.C., Bridger, G., Gutierrez-Ramos, J.C., Williams, T.J., and Rankin, S.M. (2003).

Chemokines acting via CXCR2 and CXCR4 control the release of neutrophils from the bone marrow and their return following senescence. Immunity 19, 583-593.

Massena, S., Christoffersson, G., Vagesjo, E., Seignez, C., Gustafsson, K., Binet, F., Herrera Hidalgo, C., Giraud, A., Lomei, J., Westrom, S., et al. (2015). Identification and characterization of VEGF-A-responsive neutrophils expressing CD49d, VEGFR1, and CXCR4 in mice and humans. Blood 126, 2016-2026.

Mishalian, I., Bayuh, R., Levy, L., Zolotarov, L., Michaeli, J., and Fridlender, Z.G. (2013). Tumor-associated neutrophils (TAN) develop pro-tumorigenic properties during tumor progression. Cancer Immunol Immunother $62,1745-1756$.

Monticelli, S., and Natoli, G. (2017). Transcriptional determination and functional specificity of myeloid cells: making sense of diversity. Nat Rev Immunol 17, 595-607. 
Mora-Jensen, H., Jendholm, J., Fossum, A., Porse, B., Borregaard, N., and Theilgaard-Monch, K. (2011). Technical advance: immunophenotypical characterization of human neutrophil differentiation. J Leukoc Biol $90,629-634$.

Murphy, G., Reynolds, J.J., Bretz, U., and Baggiolini, M. (1977). Collagenase is a component of the specific granules of human neutrophil leucocytes. Biochem J 162, 195-197.

Nagase, H., Miyamasu, M., Yamaguchi, M., Imanishi, M., Tsuno, N.H., Matsushima, K., Yamamoto, K., Morita, Y., and Hirai, K. (2002). Cytokine-mediated regulation of CXCR4 expression in human neutrophils. J Leukoc Biol 71, 711-717.

Nakajima, H., Watanabe, N., Shibata, F., Kitamura, T., Ikeda, Y., and Handa, M. (2006). N-terminal region of CCAAT/enhancer-binding protein epsilon is critical for cell cycle arrest, apoptosis, and functional maturation during myeloid differentiation. J Biol Chem 281, 14494-14502.

Nauseef, W.M., and Borregaard, N. (2014). Neutrophils at work. Nat Immunol 15, 602-611.

Nestorowa, S., Hamey, F.K., Pijuan Sala, B., Diamanti, E., Shepherd, M., Laurenti, E., Wilson, N.K., Kent, D.G., and Gottgens, B. (2016). A single-cell resolution map of mouse hematopoietic stem and progenitor cell differentiation. Blood 128, e20-31.

Newman, A.M., Liu, C.L., Green, M.R., Gentles, A.J., Feng, W., Xu, Y., Hoang, C.D., Diehn, M., and Alizadeh, A.A. (2015). Robust enumeration of cell subsets from tissue expression profiles. Nature methods 12 , 453\%@1548-7105.

Ng, L.G., Ostuni, R., and Hidalgo, A. (2019). Heterogeneity of neutrophils. Nature Reviews Immunology 19, 255-265.

Nicolas-Avila, J.A., Adrover, J.M., and Hidalgo, A. (2017). Neutrophils in Homeostasis, Immunity, and Cancer. Immunity 46, 15-28.

Papalexi, E., and Satija, R. (2018). Single-cell RNA sequencing to explore immune cell heterogeneity. Nat Rev Immunol 18, 35-45.

Parlato, M., Souza-Fonseca-Guimaraes, F., Philippart, F., Misset, B., Adib-Conquy, M., and Cavaillon, J.M. (2014). CD24-triggered caspase-dependent apoptosis via mitochondrial membrane depolarization and reactive oxygen species production of human neutrophils is impaired in sepsis. J Immunol 192, 2449-2459.

Paul, F., Arkin, Y., Giladi, A., Jaitin, D.A., Kenigsberg, E., Keren-Shaul, H., Winter, D., Lara-Astiaso, D., Gury, M., Weiner, A., et al. (2015). Transcriptional Heterogeneity and Lineage Commitment in Myeloid Progenitors. Cell 163, 1663-1677. 
Paul, F., Arkin, Y., Giladi, A., Jaitin, D.A., Kenigsberg, E., Keren-Shaul, H., Winter, D., Lara-Astiaso, D., Gury, M., Weiner, A., et al. (2016). Transcriptional Heterogeneity and Lineage Commitment in Myeloid Progenitors. Cell 164, 325.

Pillay, J., Kamp, V.M., van Hoffen, E., Visser, T., Tak, T., Lammers, J.W., Ulfman, L.H., Leenen, L.P., Pickkers, P., and Koenderman, L. (2012). A subset of neutrophils in human systemic inflammation inhibits T cell responses through Mac-1. J Clin Invest 122, 327-336.

Porse, B.T., Pedersen, T.A., Xu, X., Lindberg, B., Wewer, U.M., Friis-Hansen, L., and Nerlov, C. (2001). E2F repression by $\mathrm{C} / \mathrm{EBPalpha}$ is required for adipogenesis and granulopoiesis in vivo. Cell 107, 247-258.

Puellmann, K., Kaminski, W.E., Vogel, M., Nebe, C.T., Schroeder, J., Wolf, H., and Beham, A.W. (2006). A variable immunoreceptor in a subpopulation of human neutrophils. Proc Natl Acad Sci U S A 103, 1444114446.

Puga, I., Cols, M., Barra, C.M., He, B., Cassis, L., Gentile, M., Comerma, L., Chorny, A., Shan, M., Xu, W., et al. (2011). B cell-helper neutrophils stimulate the diversification and production of immunoglobulin in the marginal zone of the spleen. Nat Immunol 13, 170-180.

Qiu, X., Mao, Q., Tang, Y., Wang, L., Chawla, R., Pliner, H.A., and Trapnell, C. (2017a). Reversed graph embedding resolves complex single-cell trajectories. Nature methods 14, 979.

Qiu, X., Mao, Q., Tang, Y., Wang, L., Chawla, R., Pliner, H.A., and Trapnell, C. (2017b). Reversed graph embedding resolves complex single-cell trajectories. Nat Methods 14, 979-982.

Rice, C.M., Davies, L.C., Subleski, J.J., Maio, N., Gonzalez-Cotto, M., Andrews, C., Patel, N.L., Palmieri, E.M., Weiss, J.M., Lee, J.M., et al. (2018). Tumour-elicited neutrophils engage mitochondrial metabolism to circumvent nutrient limitations and maintain immune suppression. Nature communications 9, 5099.

Robinson, M.D., McCarthy, D.J., and Smyth, G.K. (2010). edgeR: a Bioconductor package for differential expression analysis of digital gene expression data. Bioinformatics 26, 139-140.

Rönnerblad, M., Andersson, R., Olofsson, T., Douagi, I., Karimi, M., Lehmann, S., Hoof, I., de Hoon, M., Itoh, M., Nagao-Sato, S., et al. (2014). Analysis of the DNA methylome and transcriptome in granulopoiesis reveals timed changes and dynamic enhancer methylation. Blood 123, e79-e89.

Sagiv, J.Y., Michaeli, J., Assi, S., Mishalian, I., Kisos, H., Levy, L., Damti, P., Lumbroso, D., Polyansky, L., Sionov, R.V., et al. (2015). Phenotypic diversity and plasticity in circulating neutrophil subpopulations in cancer. Cell reports 10, 562-573. 
Satake, S., Hirai, H., Hayashi, Y., Shime, N., Tamura, A., Yao, H., Yoshioka, S., Miura, Y., Inaba, T., Fujita, N., et al. (2012). C/EBPbeta is involved in the amplification of early granulocyte precursors during candidemiainduced "emergency" granulopoiesis. J Immunol 189, 4546-4555.

Scapini, P., Marini, O., Tecchio, C., and Cassatella, M.A. (2016). Human neutrophils in the saga of cellular heterogeneity: insights and open questions. Immunol Rev 273, 48-60.

Schneider, W.M., Chevillotte, M.D., and Rice, C.M. (2014). Interferon-stimulated genes: a complex web of host defenses. Annu Rev Immunol 32, 513-545.

Schuyler, Ronald P., Merkel, A., Raineri, E., Altucci, L., Vellenga, E., Martens, Joost H.A., Pourfarzad, F., Kuijpers, Taco W., Burden, F., Farrow, S., et al. (2016). Distinct Trends of DNA Methylation Patterning in the Innate and Adaptive Immune Systems. Cell reports 17, 2101-2111.

Segal, A.W. (2005). How neutrophils kill microbes. Annu Rev Immunol 23, 197-223.

Segal, B.H., Leto, T.L., Gallin, J.I., Malech, H.L., and Holland, S.M. (2000). Genetic, biochemical, and clinical features of chronic granulomatous disease. Medicine (Baltimore) 79, 170-200.

Shaul, M.E., Levy, L., Sun, J., Mishalian, I., Singhal, S., Kapoor, V., Horng, W., Fridlender, G., Albelda, S.M., and Fridlender, Z.G. (2016). Tumor-associated neutrophils display a distinct N1 profile following TGFbeta modulation: A transcriptomics analysis of pro- vs. antitumor TANs. Oncoimmunology 5, e1232221.

Silvestre-Roig, C., Hidalgo, A., and Soehnlein, O. (2016). Neutrophil heterogeneity: implications for homeostasis and pathogenesis. Blood 127, 2173-2181.

Sionov, R.V., Fridlender, Z.G., and Granot, Z. (2015). The Multifaceted Roles Neutrophils Play in the Tumor Microenvironment. Cancer microenvironment : official journal of the International Cancer Microenvironment Society $8,125-158$.

Slomiany, B.A., D'Arigo, K.L., Kelly, M.M., and Kurtz, D.T. (2000). C/EBPalpha inhibits cell growth via direct repression of E2F-DP-mediated transcription. Mol Cell Biol 20, 5986-5997.

Sorensen, O., Arnljots, K., Cowland, J.B., Bainton, D.F., and Borregaard, N. (1997). The human antibacterial cathelicidin, hCAP-18, is synthesized in myelocytes and metamyelocytes and localized to specific granules in neutrophils. Blood 90, 2796-2803.

Stuart, T., Butler, A., Hoffman, P., Hafemeister, C., Papalexi, E., Mauck, W.M., 3rd, Hao, Y., Stoeckius, M., Smibert, P., and Satija, R. (2019). Comprehensive Integration of Single-Cell Data. Cell 177, 1888-1902.e1821. Stubbington, M.J.T., Rozenblatt-Rosen, O., Regev, A., and Teichmann, S.A. (2017). Single-cell transcriptomics to explore the immune system in health and disease. Science 358, 58-63. 
Subramanian, K.K., and Luo, H.R. (2009). Non-classical roles of NADPH-oxidase dependent Reactive Oxygen Species in Phagocytes In Granulocytes: Classification, Toxic Materials Produced and Pathology, R.H.a.S.

Kohlund, ed. (Nova Science Publishers, Inc.).

Suratt, B.T., Petty, J.M., Young, S.K., Malcolm, K.C., Lieber, J.G., Nick, J.A., Gonzalo, J.A., Henson, P.M., and Worthen, G.S. (2004). Role of the CXCR4/SDF-1 chemokine axis in circulating neutrophil homeostasis. Blood 104, 565-571.

Suratt, B.T., Young, S.K., Lieber, J., Nick, J.A., Henson, P.M., and Worthen, G.S. (2001). Neutrophil maturation and activation determine anatomic site of clearance from circulation. Am J Physiol Lung Cell Mol Physiol 281, L913-921.

Tak, T., Wijten, P., Heeres, M., Pickkers, P., Scholten, A., Heck, A.J.R., Vrisekoop, N., Leenen, L.P., Borghans, J.A.M., Tesselaar, K., et al. (2017). Human CD62L(dim) neutrophils identified as a separate subset by proteome profiling and in vivo pulse-chase labeling. Blood 129, 3476-3485.

Tanji-Matsuba, K., van Eeden, S.F., Saito, Y., Okazawa, M., Klut, M.E., Hayashi, S., and Hogg, J.C. (1998). Functional changes in aging polymorphonuclear leukocytes. Circulation 97, 91-98.

Taylor, P.R., Roy, S., Leal, S.M., Jr., Sun, Y., Howell, S.J., Cobb, B.A., Li, X., and Pearlman, E. (2014). Activation of neutrophils by autocrine IL-17A-IL-17RC interactions during fungal infection is regulated by IL6, IL-23, RORgammat and dectin-2. Nat Immunol 15, 143-151.

Tcyganov, E., Mastio, J., Chen, E., and Gabrilovich, D.I. (2018). Plasticity of myeloid-derived suppressor cells in cancer. Curr Opin Immunol 51, 76-82.

Theilgaard-Monch, K., Jacobsen, L.C., Borup, R., Rasmussen, T., Bjerregaard, M.D., Nielsen, F.C., Cowland, J.B., and Borregaard, N. (2005). The transcriptional program of terminal granulocytic differentiation. Blood $105,1785-1796$.

Tirosh, I., Izar, B., Prakadan, S.M., Wadsworth, M.H., Treacy, D., Trombetta, J.J., Rotem, A., Rodman, C., Lian, C., Murphy, G., et al. (2016a). Dissecting the multicellular ecosystem of metastatic melanoma by singlecell RNA-seq. Science 352, 189-196.

Tirosh, I., Venteicher, A.S., Hebert, C., Escalante, L.E., Patel, A.P., Yizhak, K., Fisher, J.M., Rodman, C., Mount, C., Filbin, M.G., et al. (2016b). Single-cell RNA-seq supports a developmental hierarchy in human oligodendroglioma. Nature 539, 309-313.

Tirouvanziam, R., Gernez, Y., Conrad, C.K., Moss, R.B., Schrijver, I., Dunn, C.E., Davies, Z.A., Herzenberg, L.A., and Herzenberg, L.A. (2008). Profound functional and signaling changes in viable inflammatory neutrophils homing to cystic fibrosis airways. Proc Natl Acad Sci U S A 105, 4335-4339. 
Trapnell, C., Cacchiarelli, D., Grimsby, J., Pokharel, P., Li, S., Morse, M., Lennon, N.J., Livak, K.J., Mikkelsen, T.S., and Rinn, J.L. (2014). The dynamics and regulators of cell fate decisions are revealed by pseudotemporal ordering of single cells. Nature biotechnology 32, 381-386.

Tsuda, Y., Takahashi, H., Kobayashi, M., Hanafusa, T., Herndon, D.N., and Suzuki, F. (2004). Three different neutrophil subsets exhibited in mice with different susceptibilities to infection by methicillin-resistant Staphylococcus aureus. Immunity 21, 215-226.

Uhl, B., Vadlau, Y., Zuchtriegel, G., Nekolla, K., Sharaf, K., Gaertner, F., Massberg, S., Krombach, F., and Reichel, C.A. (2016). Aged neutrophils contribute to the first line of defense in the acute inflammatory response. Blood 128, 2327-2337.

Veglia, F., Perego, M., and Gabrilovich, D. (2018). Myeloid-derived suppressor cells coming of age. Nat Immunol 19, 108-119.

Velten, L., Haas, S.F., Raffel, S., Blaszkiewicz, S., Islam, S., Hennig, B.P., Hirche, C., Lutz, C., Buss, E.C., Nowak, D., et al. (2017). Human haematopoietic stem cell lineage commitment is a continuous process. Nat Cell Biol 19, 271-281.

Verbeek, W., Wachter, M., Lekstrom-Himes, J., and Koeffler, H.P. (2001). C/EBPepsilon -/- mice: increased rate of myeloid proliferation and apoptosis. Leukemia 15, 103-111.

Vono, M., Lin, A., Norrby-Teglund, A., Koup, R.A., Liang, F., and Lore, K. (2017). Neutrophils acquire the capacity for antigen presentation to memory CD4(+) T cells in vitro and ex vivo. Blood 129, 1991-2001.

Wang, Q., and Doerschuk, C.M. (2002). The signaling pathways induced by neutrophil-endothelial cell adhesion. Antioxid Redox Signal 4, 39-47.

Ward, A.C., Loeb, D.M., Soede-Bobok, A.A., Touw, I.P., and Friedman, A.D. (2000). Regulation of granulopoiesis by transcription factors and cytokine signals. Leukemia 14, 973-990.

Wipke, B.T., and Allen, P.M. (2001). Essential role of neutrophils in the initiation and progression of a murine model of rheumatoid arthritis. J Immunol 167, 1601-1608.

Witko-Sarsat, V., Rieu, P., Descamps-Latscha, B., Lesavre, P., and Halbwachs-Mecarelli, L. (2000).

Neutrophils: molecules, functions and pathophysiological aspects. Lab Invest 80, 617-653.

Wu, Z., Liang, R., Ohnesorg, T., Cho, V., Lam, W., Abhayaratna, W.P., Gatenby, P.A., Perera, C., Zhang, Y., Whittle, B., et al. (2016). Heterogeneity of Human Neutrophil CD177 Expression Results from CD177P1 Pseudogene Conversion. PLoS genetics 12, e1006067. 
Yamanaka, R., Barlow, C., Lekstrom-Himes, J., Castilla, L.H., Liu, P.P., Eckhaus, M., Decker, T., WynshawBoris, A., and Xanthopoulos, K.G. (1997). Impaired granulopoiesis, myelodysplasia, and early lethality in CCAAT/enhancer binding protein epsilon-deficient mice. Proc Natl Acad Sci U S A 94, 13187-13192.

Yu, G., Wang, L.G., Han, Y., and He, Q.Y. (2012). clusterProfiler: an R package for comparing biological themes among gene clusters. OMICS 16, 284-287.

Yvan-Charvet, L., and Ng, L.G. (2019). Granulopoiesis and Neutrophil Homeostasis: A Metabolic, Daily Balancing Act. Trends Immunol 40, 598-612.

Zhang, D.-E., Zhang, P., Wang, N.-d., Hetherington, C.J., Darlington, G.J., and Tenen, D.G. (1997). Absence of granulocyte colony-stimulating factor signaling and neutrophil development in CCAAT enhancer binding protein $\alpha$-deficient mice. Proceedings of the National Academy of Sciences 94, 569-574.

Zhang, D., Chen, G., Manwani, D., Mortha, A., Xu, C., Faith, J.J., Burk, R.D., Kunisaki, Y., Jang, J.-E., Scheiermann, C., et al. (2015). Neutrophil ageing is regulated by the microbiome. Nature 525, 528-532. Zhang, X., Kluger, Y., Nakayama, Y., Poddar, R., Whitney, C., DeTora, A., Weissman, S.M., and Newburger, P.E. (2004). Gene expression in mature neutrophils: early responses to inflammatory stimuli. J Leukoc Biol 75 , $358-372$.

Zhou, G., Yu, L., Fang, L., Yang, W., Yu, T., Miao, Y., Chen, M., Wu, K., Chen, F., Cong, Y., et al. (2018). $\mathrm{CD} 177(+)$ neutrophils as functionally activated neutrophils negatively regulate IBD. Gut 67, 1052-1063.

Zhu, Y.P., Padgett, L., Dinh, H.Q., Marcovecchio, P., Blatchley, A., Wu, R., Ehinger, E., Kim, C., Mikulski, Z., Seumois, G., et al. (2018). Identification of an Early Unipotent Neutrophil Progenitor with Pro-tumoral Activity in Mouse and Human Bone Marrow. Cell reports 24, 2329-2341.e2328.

Zilionis, R., Engblom, C., Pfirschke, C., Savova, V., Zemmour, D., Saatcioglu, H.D., Krishnan, I., Maroni, G., Meyerovitz, C.V., Kerwin, C.M., et al. (2019). Single-Cell Transcriptomics of Human and Mouse Lung Cancers Reveals Conserved Myeloid Populations across Individuals and Species. Immunity 50, 1317 1334.e1310. 


\section{Figure legends}

Figure 1. Single-cell RNA-seq analysis of steady-state bone marrow (BM), peripheral blood (PB), and spleen (SP) neutrophils.

(A) Overview of study design.

(B) Uniform manifold approximation and projection (UMAP) of 12,285 neutrophils from BM, PB, and SP colored by cell type, sample origin, and inferred cluster identity, respectively. MP: myeloid progenitors. Cont: the contaminating population mainly consisted of low-quality cells so was discarded from further analysis. GM: low-quality G3-like cells (low UMI count per cell, low UMI count per gene, and high percentage of mitochondrial UMI counts). Similarly, unless otherwise stated, GM were excluded from all other downstream analyses.

(C) Proportions of the nine neutrophil clusters in four samples.

(D) Heatmap showing row-scaled expression of the 20 highest differentially expressed genes (DEGs, Bonferroni-corrected P-values $<0.05$, Student's t-test) per cluster for all neutrophils except cells from the GM population.

(E) Dot plot showing scaled expression of selected signature genes for each cluster colored by average expression of each gene in each cluster scaled across all clusters. Dot size represents the percentage of cells in each cluster with more than one read of the corresponding gene.

(F) Gene ontology (GO) analysis of DEGs for each cluster. Selected GO terms with Benjamini-Hochbergcorrected P-values $<0.05$ (one-sided Fisher's exact test) are shown and colored by gene ratio.

(G) Unsupervised hierarchical clustering of the eight clusters based on the average gene expression of cells in each cluster. Organ distribution of each cluster is shown on the left.

(H) Monocle trajectories of neutrophils colored by sample origin (left) and cluster identity (right). Each dot represents a single cell. Cell orders are inferred from the expression of the most variable genes across all cells. Trajectory directions were determined by biological prior.

(I) Heatmap showing row-scaled expression of cell cycle-related genes for G0-G4 neutrophils.

(J) Violin plot of maturation scores for each cluster. 
A

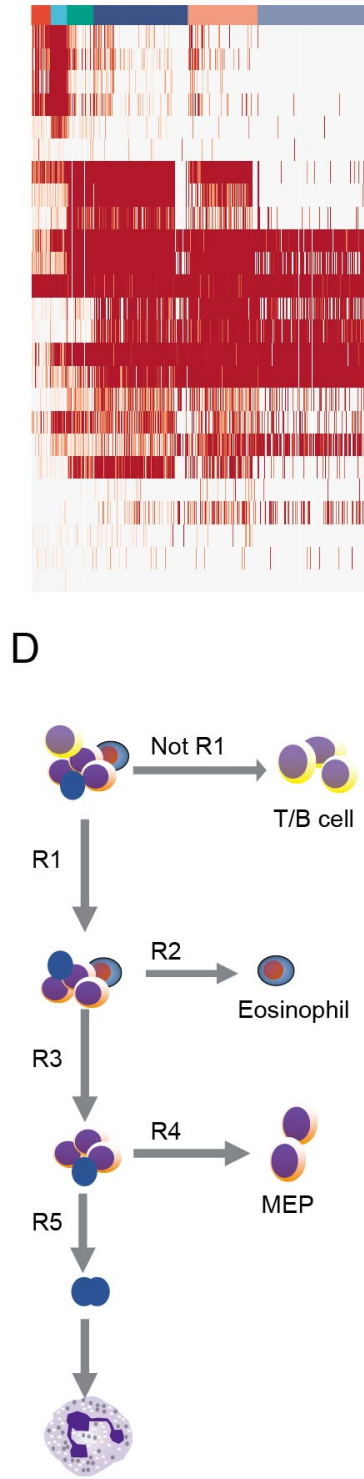

B
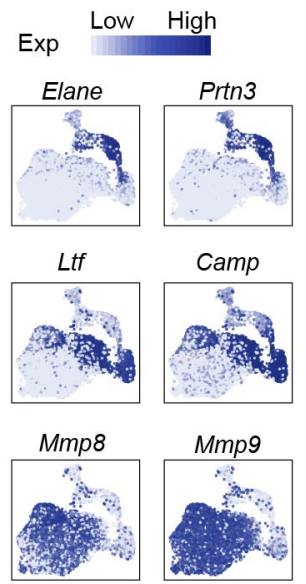

C
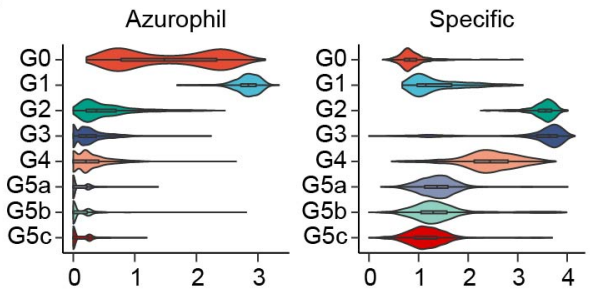

Gelatinase

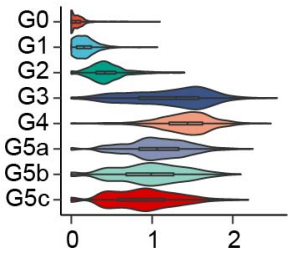

Secretory vesicle

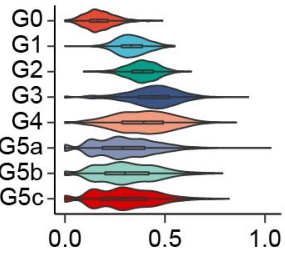

F
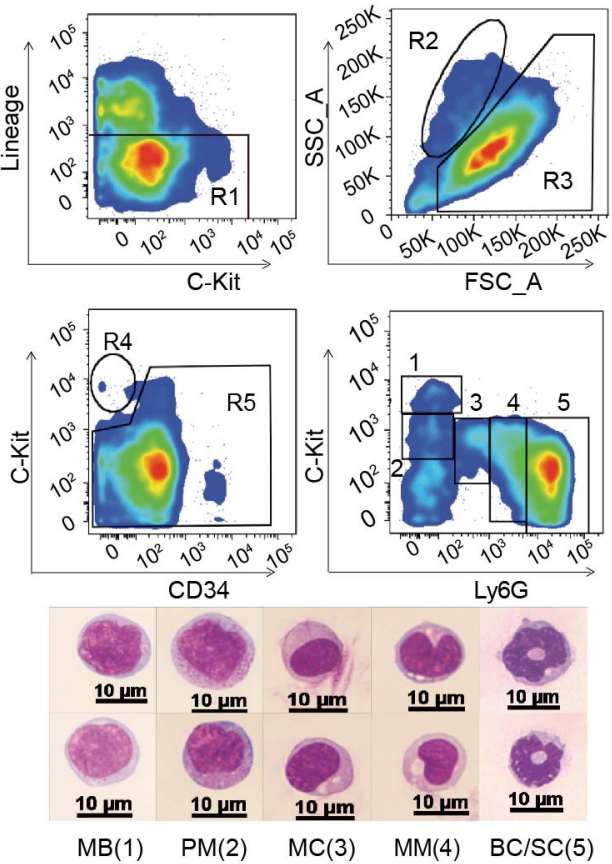

E
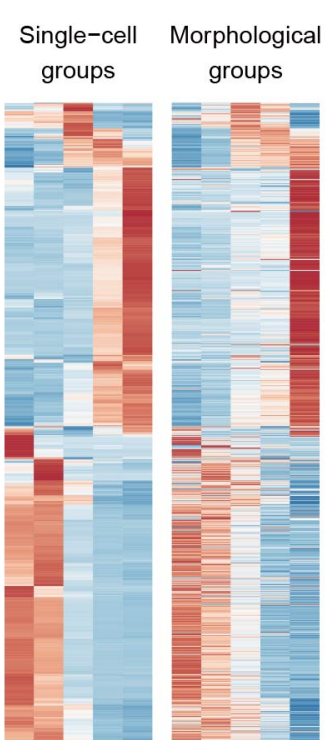

이웡엉
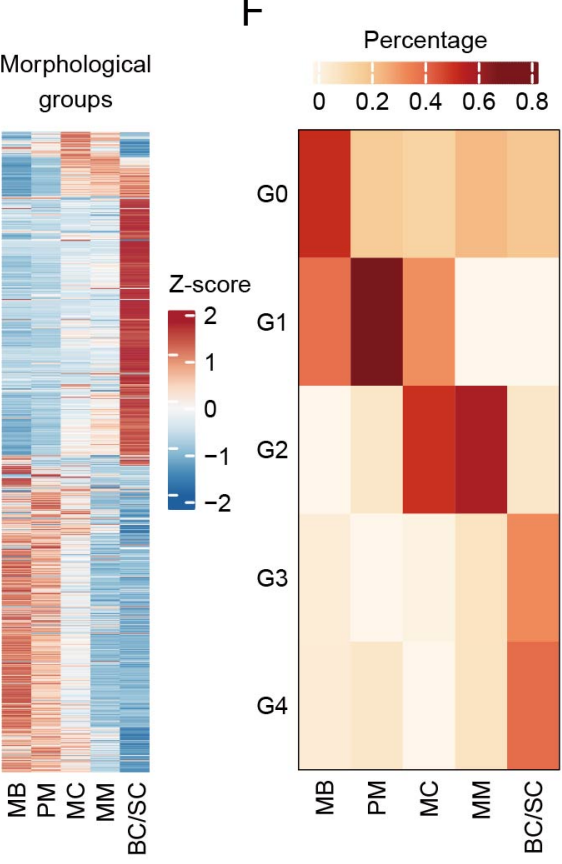

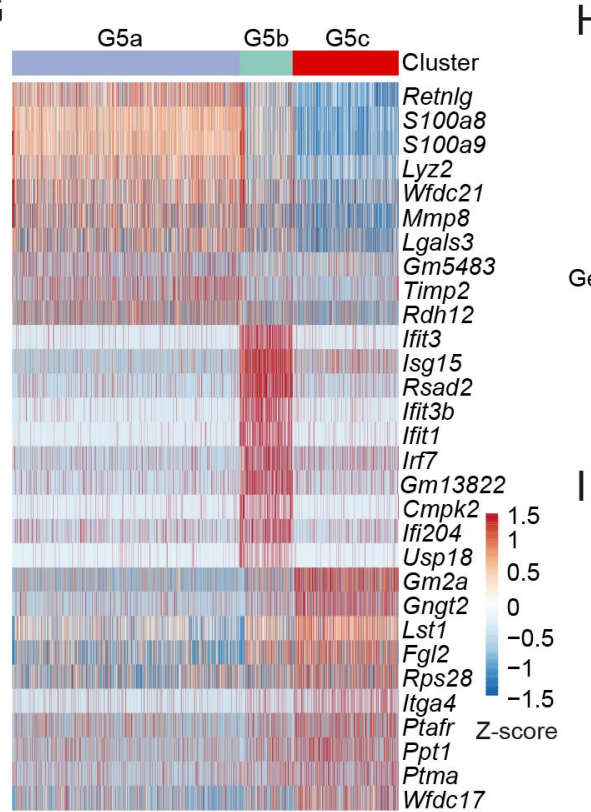

J
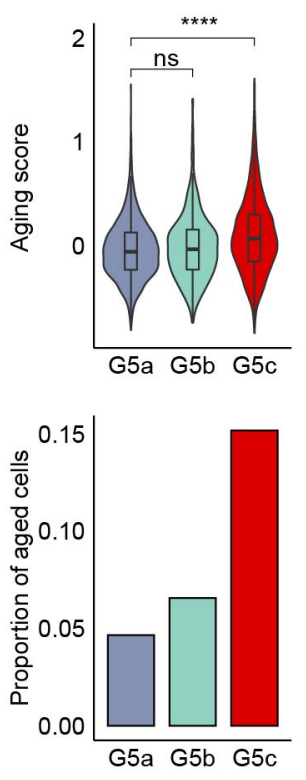


\section{Figure 2.}

\section{(A-F) Transcriptional landscape of neutrophils along differentiation and maturation trajectories.}

(A) Heatmap showing expression of neutrophil granule-related genes for all neutrophils.

(B) Expression of six typical neutrophil granule genes.

(C) Violin plots of azurophil score, specific score, gelatinase score, and secretory score for each cluster.

(D-F) scRNA-seq-defined differentiating neutrophil populations correlated with classical morphology-defined neutrophil subpopulations.

(D) FACS sorting and staining of five mouse BM neutrophil populations for bulk sequencing. Left: Gating diagram. R1 (CD4- CD8a-CD45R/B220-Ter119-) was selected, R2 (eosinophil) and R4 (MEP) were excluded, and remaining R5 (neutrophils) were selected. Top right: Same R1-R5 from (left) but showing FACS gating of five detailed neutrophil subpopulations and the morphology of the sorted cells, among which: 1 was c-

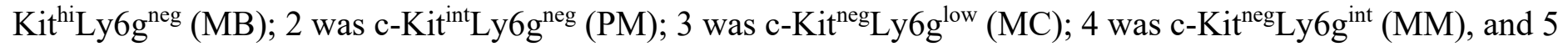
was c-Kit'Ly6ghi (BC/SC). Bottom right: Representative Wright-Giemsa staining of these populations (scale bar represents $10 \mu \mathrm{m}$ ); Data are representative of three independent experiments.

(E) Heatmaps showing row-scaled expression of scRNA-seq-defined DEGs across averaged single-cell groups (left) and morphological groups (right). Only genes detected in both scRNA-seq data and bulk RNA-seq data are visualized.

(F) Coefficient matrix showing deconvolution results of morphological bulk profiles. The 20 highest DEGs per single-cell group were selected as signatures for deconvolution. Each column is normalized by column sums.

\section{(G-H) Transcriptional landscape of mature neutrophils in the peripheral blood and spleen.}

(G) Heatmap showing row-scaled expression of the ten highest DEGs per cluster for G5a, G5b, and G5c neutrophils.

(H) Gene ontology (GO) analysis of DEGs for each of the three G5 clusters. Selected GO terms with Benjamini-Hochberg-corrected P-values $<0.05$ (one-sided Fisher's exact test) are shown.

\section{(I-J) Expression of neutrophil aging signatures.}

(I) Heatmap showing row-scaled expression of aging-related genes for all neutrophils.

(J) Top: Violin plot of aging score defined as weighted average Z-scores of aging-related genes for the three G5 clusters. Bottom: Proportions of aged cells in each G5 clusters. 
A

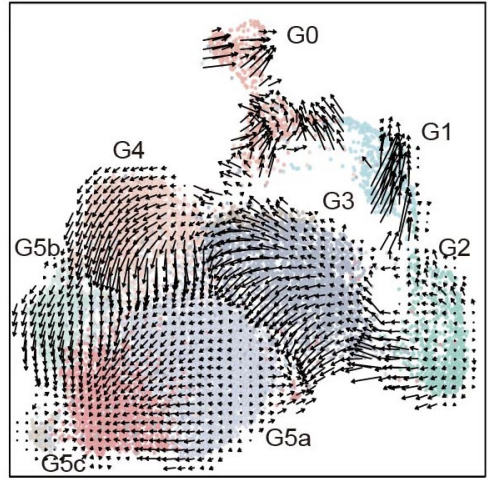

B

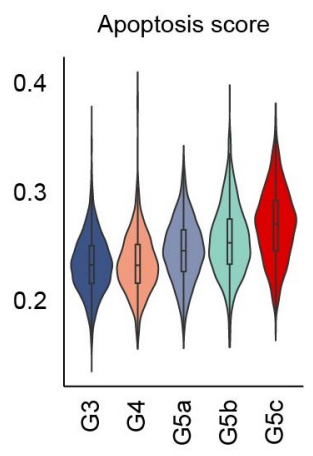

C

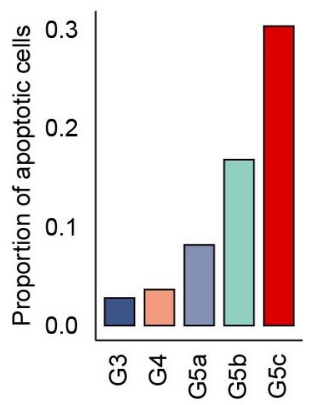

D

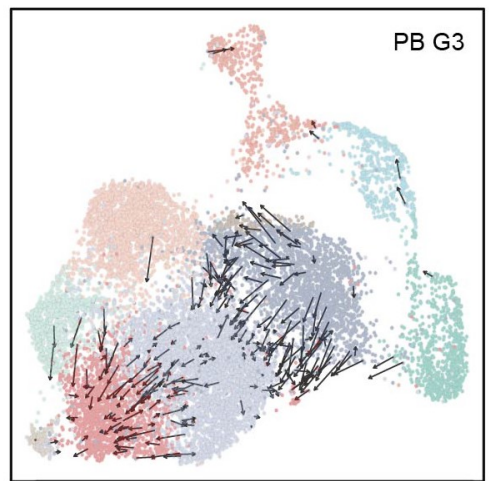

J

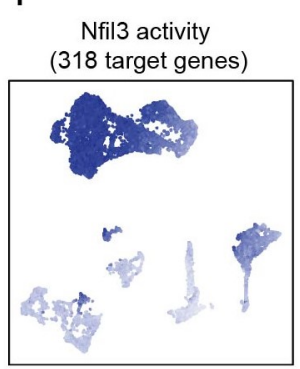

$\mathrm{F}$

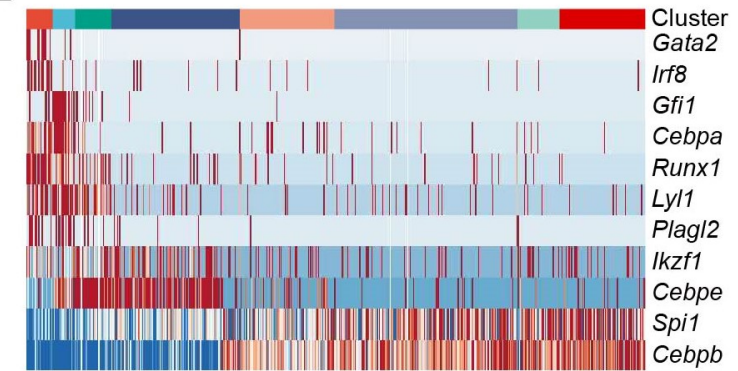

Cluster $\bullet \mathrm{R} 1 \quad \bullet \mathrm{R} 3 \quad \bullet \mathrm{R} 5 \quad \bullet \mathrm{R} 7$

G
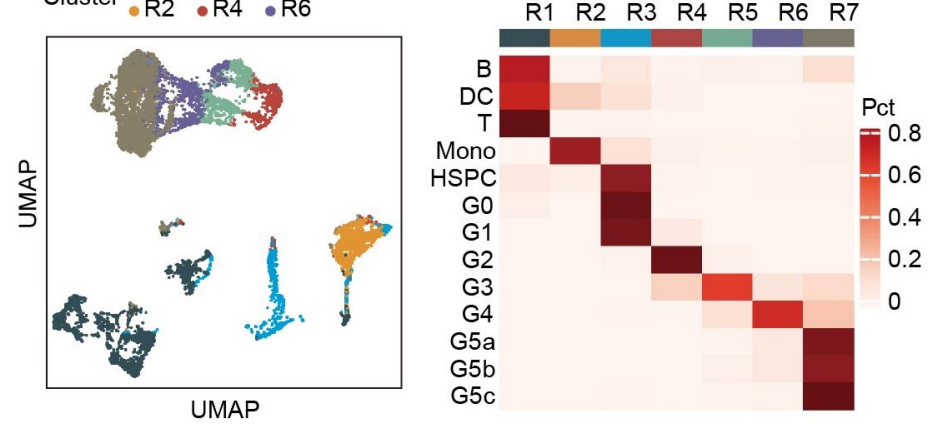

$\mathrm{H}$

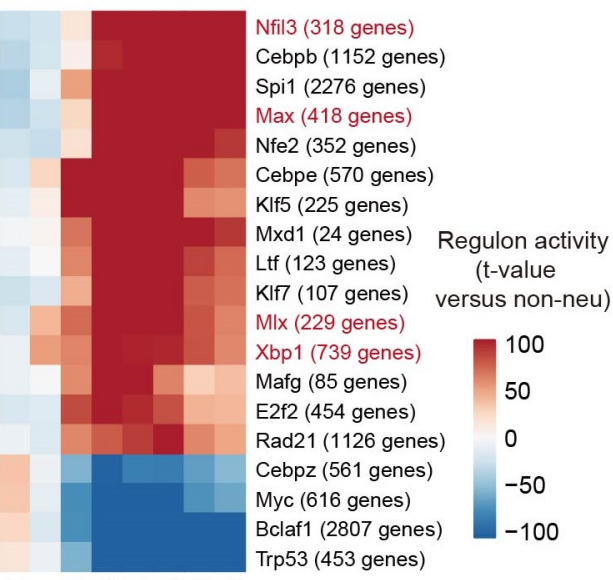

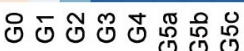

Xbp1 activity
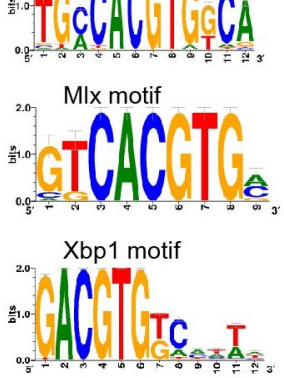

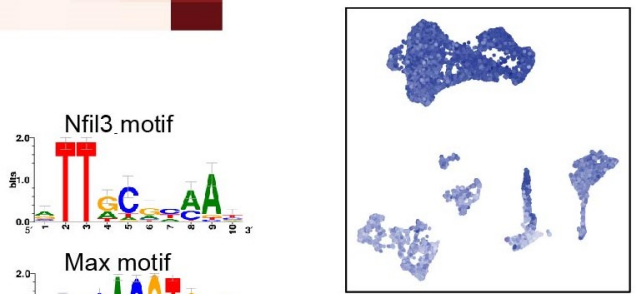

Max activity

(418 target genes)

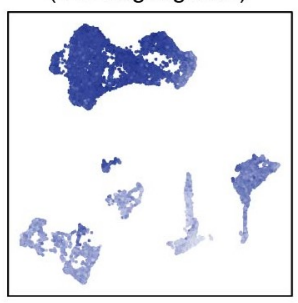

Mlx activity

(229 target genes)

(739 target genes)

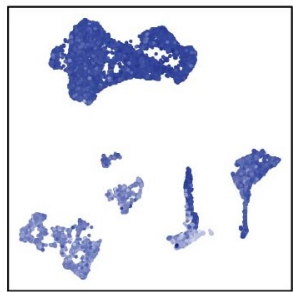
(t-value

versus previous stage)
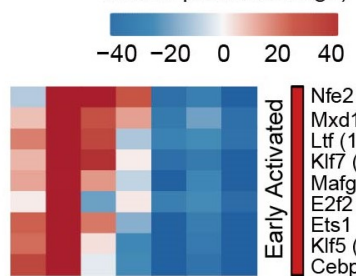

D.
Mfe2 (352 genes)
Mxd1 (24 genes)
Ltf (123 genes)
Klf7 (107 genes)
Mafg (85 genes)
E2f2 (454 genes)
Ets1 (600 genes)
Klf5 (225 genes)
Cebpe (570 genes)

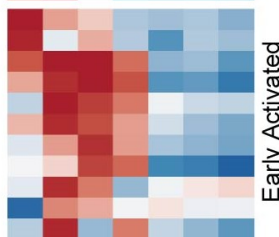

Mlx (229 genes)

Mlx (229 genes) Xbp1 (739 genes) Max (418 genes) Max (418 genes) Klf13 (551 genes) Zbtb7b (147 genes) Zfp595 (31 genes) Etv5 (73 genes) Tc77 (76 genes)

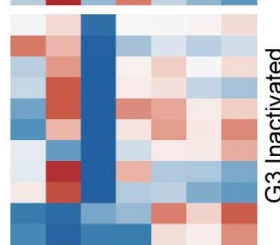

E2f1 (548 genes) Nelfe (559 genes) Pou2af1 (40 genes) Rb1 (179 genes) Rux1 (122 genes) Cux1 (122 genes) Ezh2 (2507 genes) Nfyb (332 genes) E2f8 (615 genes) Bclaf1 (2807 genes)
Trp53 (453 genes)

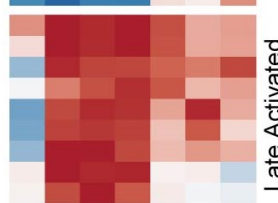

Cebpp (1152 genes) Nfil3 (318 genes) Tbx6 (27 genes) Irf9 (399 genes) IIf2 (521 genes) Irt2 (521 genes) irf5 (472 genes) Stat3 (415 genes)

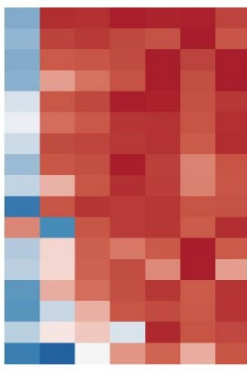
Irf7 (822 genes) Stat1 (630 genes) $\mathrm{Nfkb} 2$ (255 genes) Atf3 (207 genes) Fosl2 (321 genes) Ddit3 (180 genes) KIf3 (129 genes) Junb (675 genes) Jdp2 (36 genes) Irf8 (992 genes) $\mathrm{Nfe} 2 \mathrm{l} 2$ (229 genes) Irf1 (380 genes) Stat2 (359 genes) Fosb (526 genes)

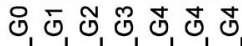
Mgr (16 genes)
Myc (616 genes) Fosl1 (12 genes) Ybx1 (1142 genes) KIf2 (603 genes) Jund (838 genes) Pgr (16 genes) 


\section{Figure 3.}

\section{(A-D) The origin and inter-relationship of neutrophil subpopulations.}

(A) Velocity analysis reveals the origin and inter-relationship of neutrophil subpopulations. Velocity fields were projected onto the UMAP plot.

(B) Violin plot of apoptosis scores (GO:0097193) for G3-G5 clusters.

(C) Proportions of apoptotic cells in each cluster identified by a two-component Gaussian mixture model.

(D) As in (A) but only of G3 neutrophils originating from PB.

\section{(E-J) The formation of neutrophil subpopulations is driven by both known and a large set of uncharacterized transcription factors.}

(E) Heatmap showing row-scaled gene expression of TFs known to be involved in granulopoiesis and neutrophil function.

(F) UMAP of the regulon activity matrix of neutrophils and 7209 non-neutrophils under normal conditions. Kmeans clustering was performed on the first 20 principal components (PCs) of the regulon activity matrix with cluster number $k=7$. Each cell is assigned the color of its K-means cluster.

(G) Confusion matrix showing the percentage overlap of Seurat transcriptome-based clusters with K-means regulon-based clusters.

(H) Heatmap of the t-values of regulon activity derived from a generalized linear model of the difference between cells from one neutrophil subpopulation and cells from other non-neutrophil populations. Only regulons with at least one absolute t-value $>100$ are visualized. Previously uncharacterized neutrophil-specific transcription factors are marked in red with binding motif shown on the right.

(I) Activities of the four newly identified neutrophil-specific regulons.

(J) As in (H), but t-values representing activity change between the current developmental stage and the previous one. Only regulons with at least one absolute t-value $>40$ are visualized. Regulons are hierarchically clustered based on activation pattern (red and orange: early-activated, yellow: G3-inactivated, green: lateactivated, blue: global-activated). 
A

Control $\bullet \mathrm{G} 0 \bullet \mathrm{G} 1 \cdot \mathrm{G} 2 \cdot \mathrm{G} 3 \cdot \mathrm{G} 4$ $\mathrm{G} 5 \mathrm{a} \bullet \mathrm{G} 5 \mathrm{~b} \bullet \mathrm{G} 5 \mathrm{c} \bullet \mathrm{GM}$

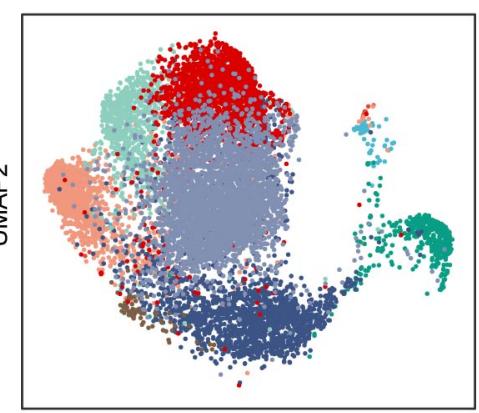

UMAP1

C $\begin{array}{lllll}\text { Z-score } & & & \\ 2 & 1 & 0 & -1 & \text { expression }\end{array}$ $\begin{array}{lllllll}2 & 1 & 0 & -1 & 0255075100 \\ & & & \bullet\end{array}$

Cd34-

Sox4- -

Rpl12-000000000....

Elane- $00000 \cdot 0 \cdot$

Prtn3-0000०0.0.

Mpo-000०००...

Chil3-0000000000.................. Tuba1b-000000.

Fcnb-. ०००००。

Ltf- 000000000

Ngp-0000000000......

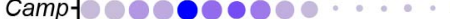
$m t-C 01-0000000000000000$ $m t-C 03-00000000000000 ० \mathrm{C}$ $m t-$ Co2Retnlg-0000000000000000 Mmp8- 00000000000000 $\mathrm{CxCl} 2 \ldots . . . . . . .$. Ccl6- 000000.00000000 Gm5483 . . . . . . . Stfa2l1 ….... Isg15 ….....

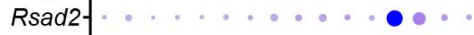

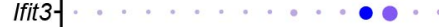
Fgl2 Gm2a Gngt2- …...... E.coli $-+\mathbf{+}+\mathbf{+}+-+++-+-+-+$

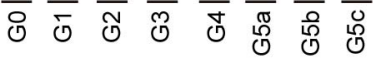
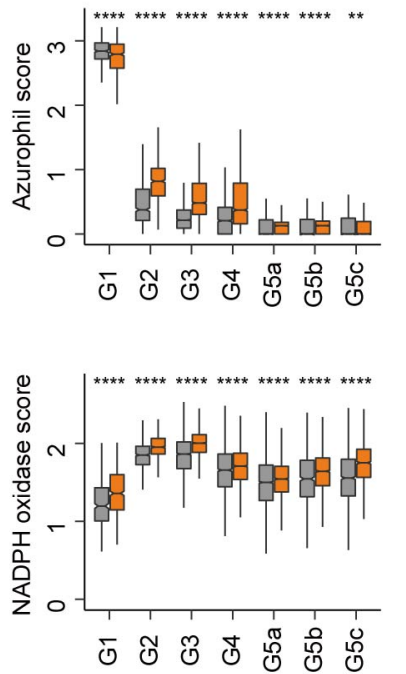

E.coli (24h) $\bullet \mathrm{GO} \bullet \mathrm{G} 1 \bullet \mathrm{G} 2 \bullet \mathrm{G} 3$

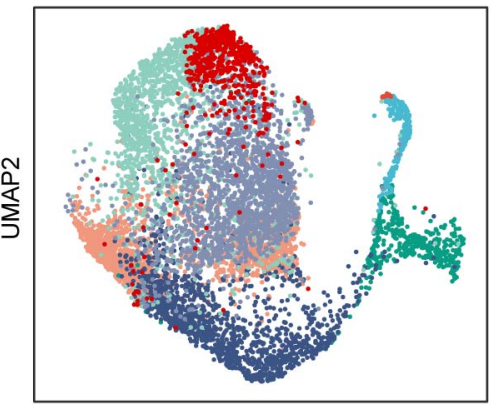

UMAP1

D

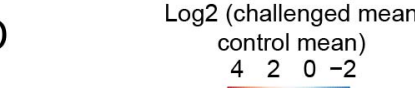
$\begin{array}{llll}4 & 2 & 0 & -2\end{array}$

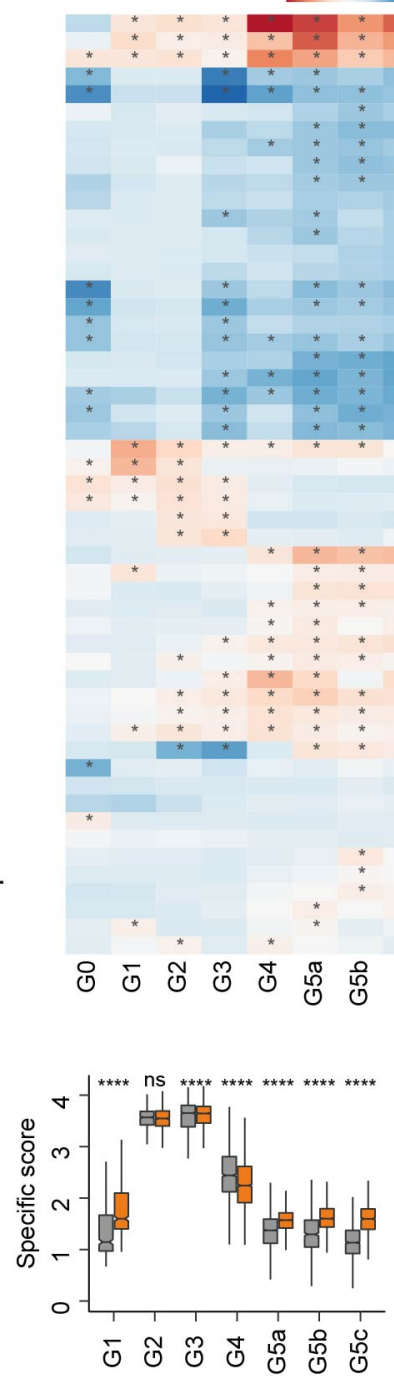

Wfdc17 Iftitm III 16
Ccl6
Mpc1 Btg2
Thibs1
Gm10076
Glipr1 Glipr1
Cytip
H2-Q10
Hist 1 1h1c $\mathrm{S} / \mathrm{l} 25 \mathrm{25} 3 \mathrm{3}$
Arrdc 3

Fos

Btg1

2310001 H17Rik Amica1
Gm9843 $\mathrm{Fg} / 2$
$\mathrm{Rps} 2 \mathrm{~g}$ rt Ifitm
Saa3
Hspa5 Calr SCtfa1 lgfbp6 6 Wfdc21 Wrdc2 Gyg Plac8
Prok2 Prok2
Tspo

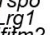
Lybo Nfkbia Xist Taglin2 Lybi
Zfp36/1
Ctsb Asnsd
Chil3
Ly6e Ly6 (1)

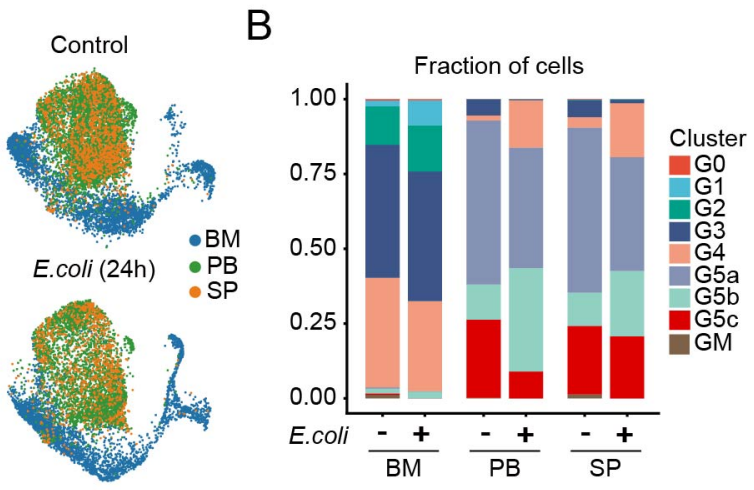

E

G0-G4 Gene ontology

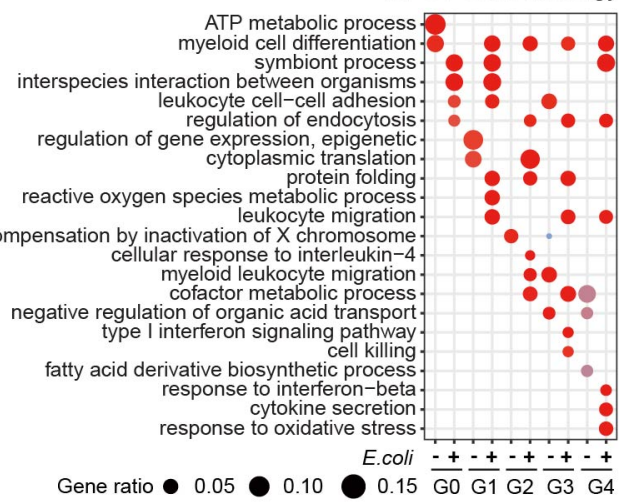

G5 Gene ontology

regulation of cellular amideplasmic translation metabolic process leukocyte migration. response to interferon-beta.

apoptotic signaling pathway in response to DNA damage. positive regulation of cytokine production. regulation of inflammatory response. celluar response to oxidative stress.
regulation of MHC class II biosynthetic process. regulation of cell shape
ERK1 and ERK2 cascade. cellular response to reactive oxygen species. interleukin-6 secretion.

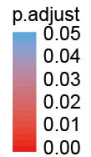

positive regulation of cytokine secyetesion. response to interferon-gamma cellular response to metal ion

cytokine-mediated signaling pathway response to molecule of bacterial origin.

cell killing -

E.coli $-+-+\div$

Gene ratio $\bullet 0.05 \bullet 0.10 \overline{\text { G5a }} \overline{\text { G5b }} \overline{\text { G5c }}$

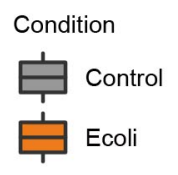

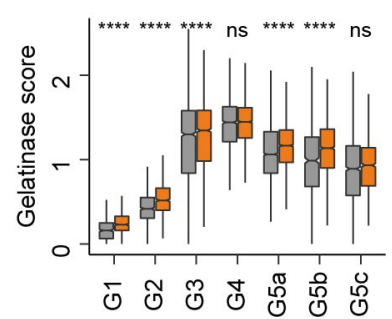

$\mathrm{H}$

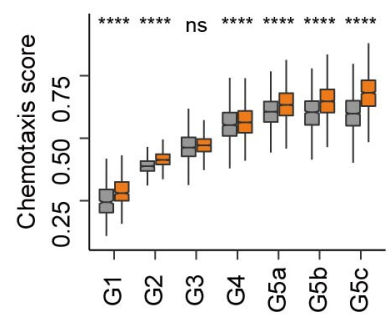

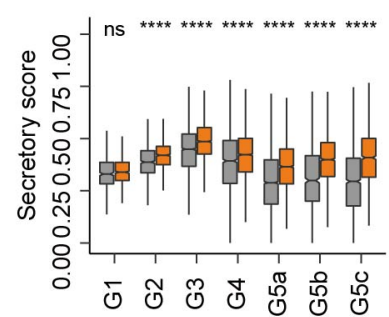

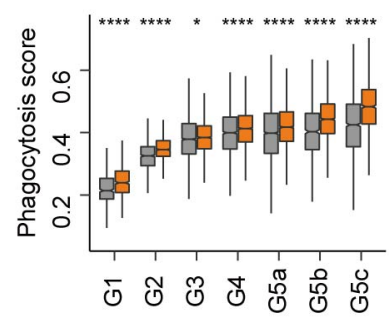




\section{Figure 4. Bacterial infection primes neutrophils for augmented functionality without affecting their overall heterogeneity.}

(A) Comparison of control and E. coli-challenged neutrophils originating from BM, PB, and SP. All neutrophils under both control and E. coli-challenged conditions $(13,687$ cells) are projected together by UMAP but are displayed separately by experimental condition.

(B) Comparisons of neutrophil composition between control and E. coli challenge in BM, PB, and SP before and after E. coli challenge.

(C) Dot plot showing scaled expression of signature genes for each cluster before and after E. coli challenge colored by average expression of each gene in each cluster scaled across all clusters. Dot size represents the percentage of cells in each cluster with more than one read of the corresponding gene.

(D) Heatmap showing $\log _{2}$ (fold-change) in gene expression of the representative cluster-based DEGs between control and E. coli-challenged neutrophils. The asterisks mean $\log _{2}$ (fold-change) $>1$ in corresponding cells.

(E) GO analysis of cluster-based DEGs between control and E. coli-challenged neutrophils. Selected GO terms with Benjamini-Hochberg-corrected P-values $<0.05$ (one-sided Fisher's exact test) are shown.

(F-H) Comparisons of functional scores between control and E. coli-challenged neutrophils for each cluster. (F): Granule scores; $(\mathrm{G})$ : NADPH oxidase complex score; $(\mathrm{H})$ : Chemotaxis score and phagocytosis score. ns, $\mathrm{P}>0.05 ; *, \mathrm{P}<=0.05 ; * *, \mathrm{P}<=0.01 ; * * *, \mathrm{P}<0.001, * * * *, \mathrm{P}<=0.0001$. student's t-test. 
A

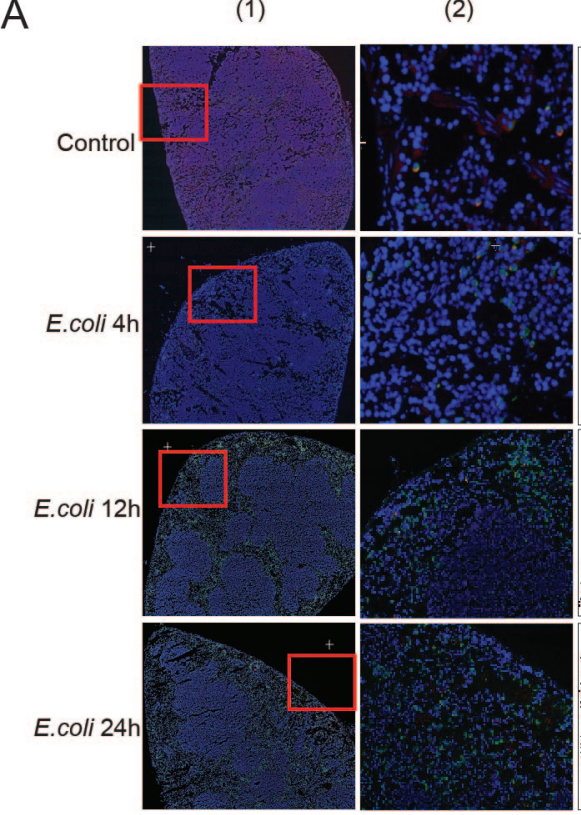

(3)

S100a8
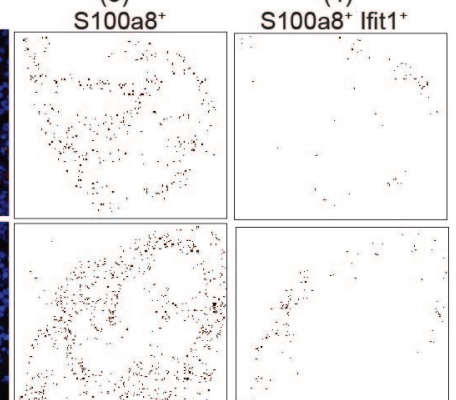

S100a8
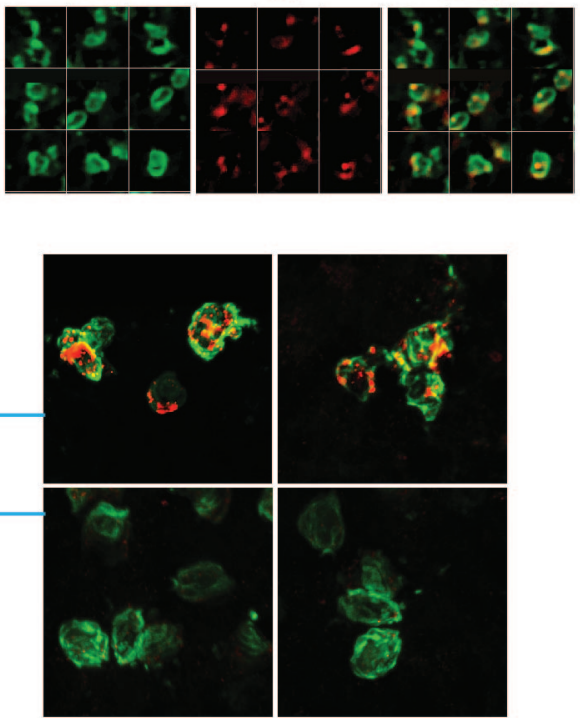

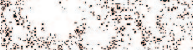

B
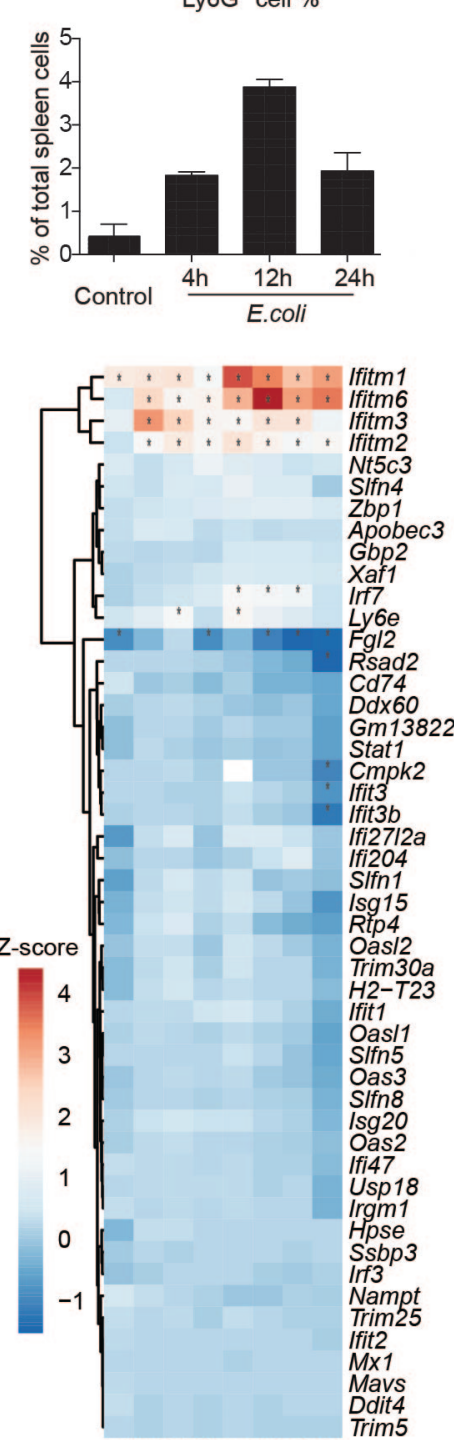

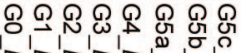

m!m!m!m $m$

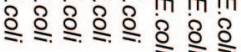

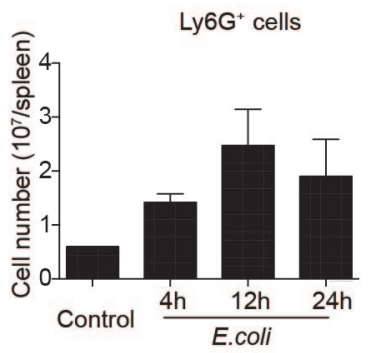

D
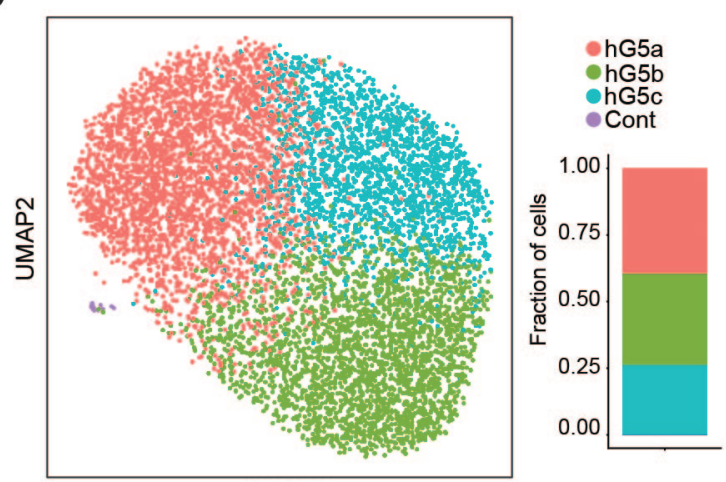

E

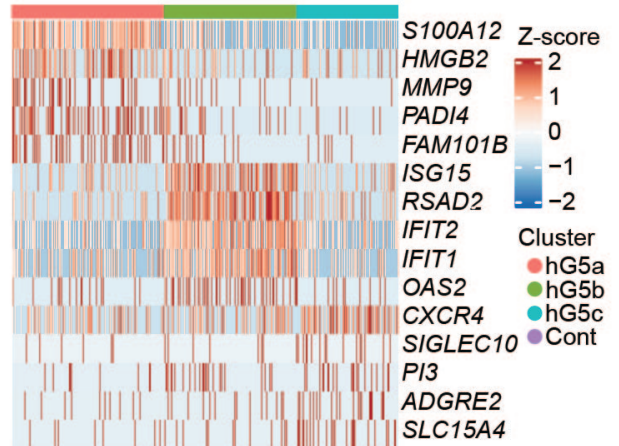

G5b cells
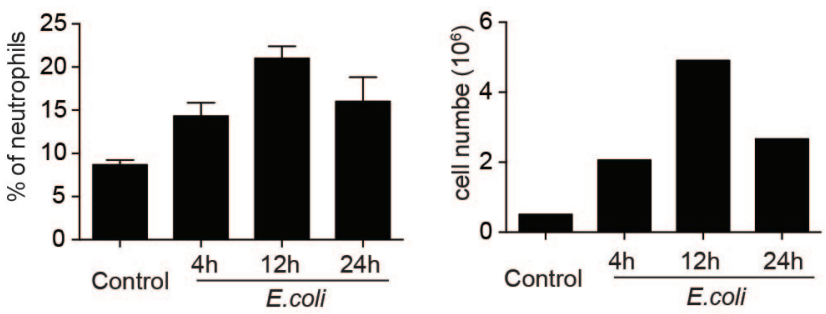

F

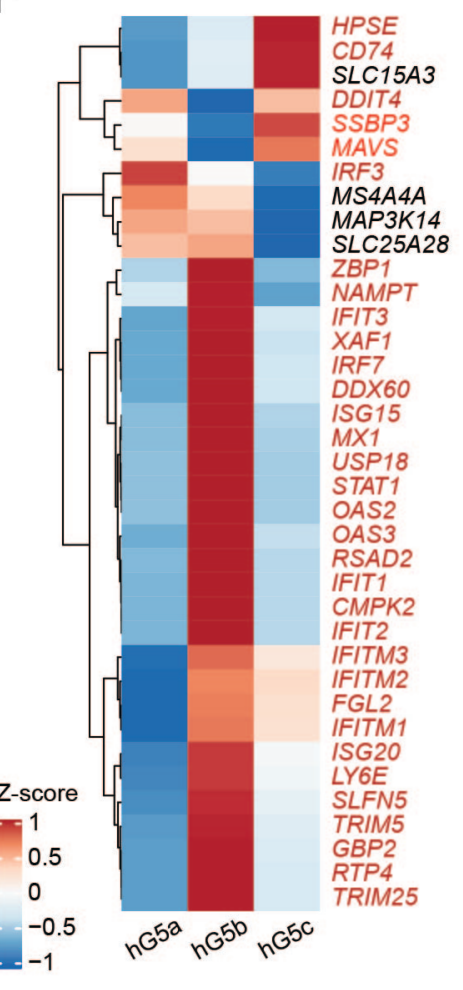




\section{Figure 5. The ISG-expressing neutrophil population is present in both humans and mice and expands during bacterial infection.}

(A) Quantitative image analysis of the spatial distribution of G5b in whole spleen sections. Left: Laser scanning cytometry (LSC) image analysis of the whole spleen. (1) Low-magnification image of axial spleen cryosection immunostained for DAPI (blue), S100a8 (green), and Ifit1 (red). (2) Representative images of spleens from the selected region from (1). (3) Localization diagram of $\mathrm{S} 100 \mathrm{a} 8^{+}$cells in the whole spleen from (1). Cells gated positive based on the fluorescence intensity in the S100a8 channel. Each dot represents a single cell. (4) Localization diagram of S100a8 ${ }^{+} \mathrm{Ifit}^{+}(\mathrm{G} 5 \mathrm{~b})$ cells gated from (3). Each dot represents a single cell. Right: LSC images (top) and confocal images (bottom) of representative G5b neutrophils.

(B) Quantification and relative frequency of spleen $\mathrm{Ly}_{6 \mathrm{~g}}{ }^{+}$cells (left, measured by FACS) and spleen G5b cells (right, S100a $8^{+} \mathrm{Ifit}^{+}$, measured by LSC) at different time points after E. coli infection. Results are the mean \pm SD of three independent experiments.

(C) Heatmap showing $\log _{2}$ fold change in expression of 49 ISGs before and after E. coli challenge for each cluster. Genes are marked with an asterisk if their expression changed significantly as identified by a Student's t-test (Bonferroni-corrected P-value $<0.05$ ).

(D) UMAP of neutrophils from human peripheral blood (PB) colored by cluster identity. The fraction of cells in each cluster is displayed on the right.

(E) Heatmap showing row-scaled expression of the five highest DEGs (Bonferroni-corrected P-values $<0.05$, Student's t-test) per cluster for all hG5 neutrophils.

(F) Heatmap showing expression of 37 ISGs for the three human neutrophil clusters. Genes marked in red are conserved across mouse and human. 
A

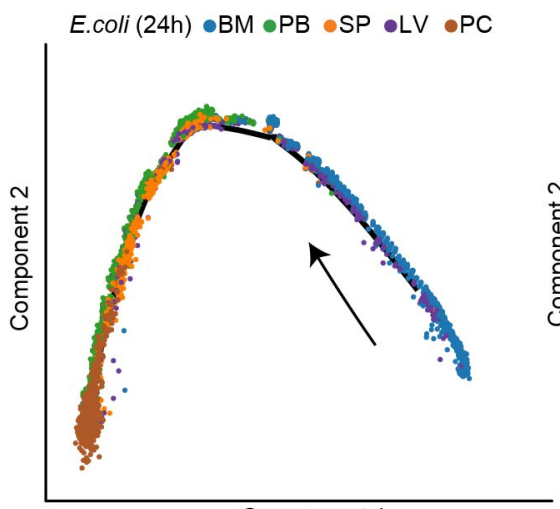

Component 1
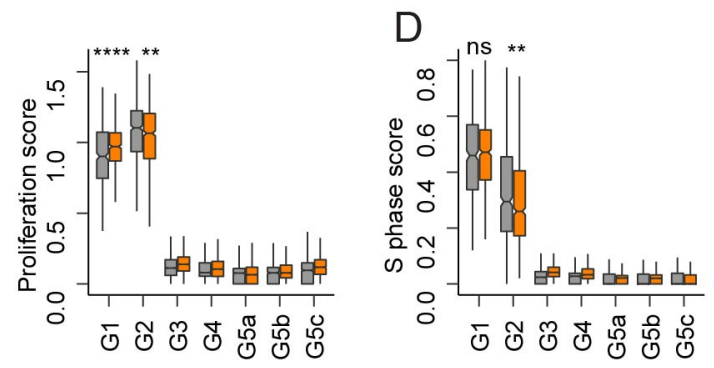

F

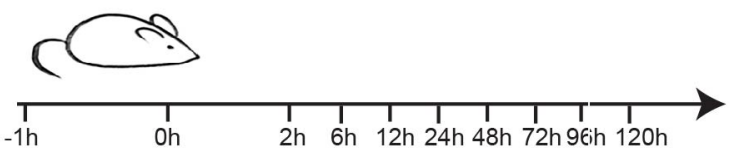

Edu

(i.p. injection) (i.p. $1 \times 10^{7 /}$ mouse)

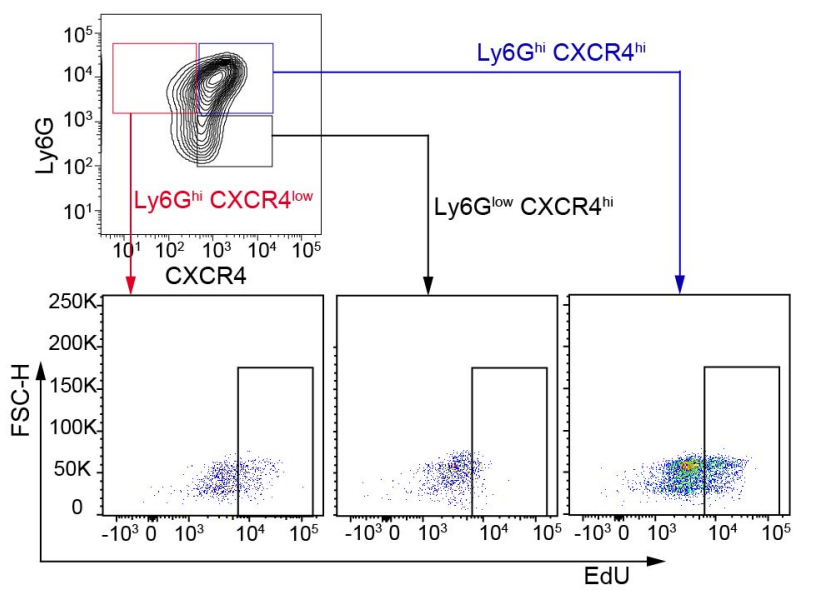

B

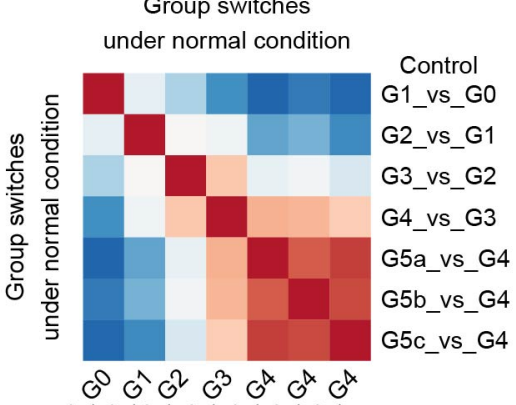

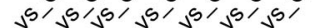

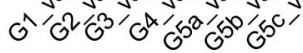

Pearson correlation

Group switches under normal condition

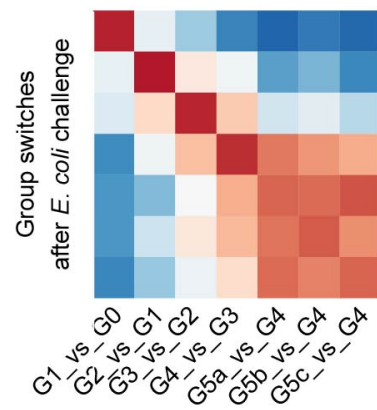

G1_vs_G0 (E.coli) G2_vs_G1 (E.coli) G3_vs_G2 (E.coli) G4_vs_G3 (E.coli) G5a_vs_G4(E.coli) G5b_vs_G4(E.coli) G5c_vs_G4 (E.coli)

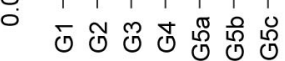
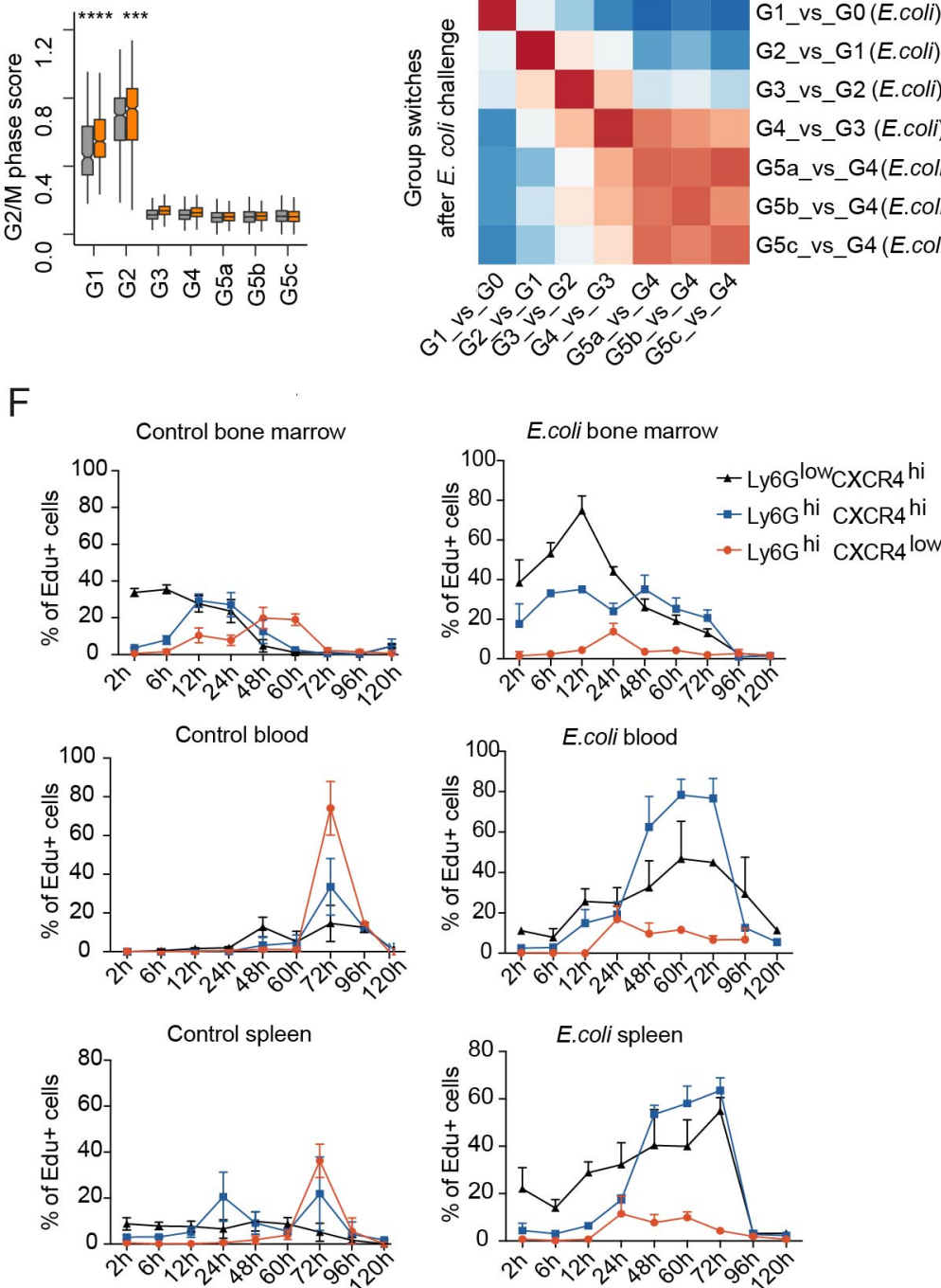


\section{Figure 6. Bacterial infection accelerates G1 cell division and post-mitotic maturation without altering overall neutrophil differentiation programs.}

(A) Monocle trajectories of E. coli-challenged neutrophils colored by sample origin (left) and cluster identity (right). Each dot represents a single cell. Cell orders are inferred from the expression of the most variable genes across all cells. The trajectory direction was determined by biological prior.

(B) Correlation matrices of t-values for regulon activity change during each group transition event under normal conditions (top) or after E. coli challenge (bottom). For each group transition event after challenge, the direct comparison to all normal transition events is demonstrated (bottom).

(C-D) Comparisons of proliferation score (C), S-phase score, and G2M score (D) between control and E. colichallenged neutrophils for each of the 8 clusters.

(E-F) In vivo EdU incorporation assay. (E) Top: Schematic. Bottom: Gating strategy of the three neutrophil subpopulations: immature (Ly6g ${ }^{\text {low }} \mathrm{Cxcr} 4{ }^{\text {hi }}$, black), intermediate (Ly6ghi $\mathrm{Cxcr} 4^{\text {hi }}$, blue), and mature

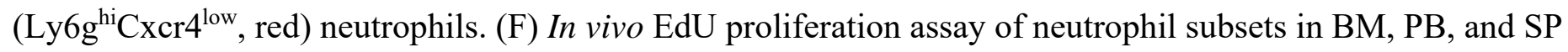
at sequential time points with or without E. coli challenge. Data are represented as percentages of EdU ${ }^{+}$cells in the corresponding gated subpopulation. Results are the mean $( \pm \mathrm{SD})$ of three independent experiments. 
A
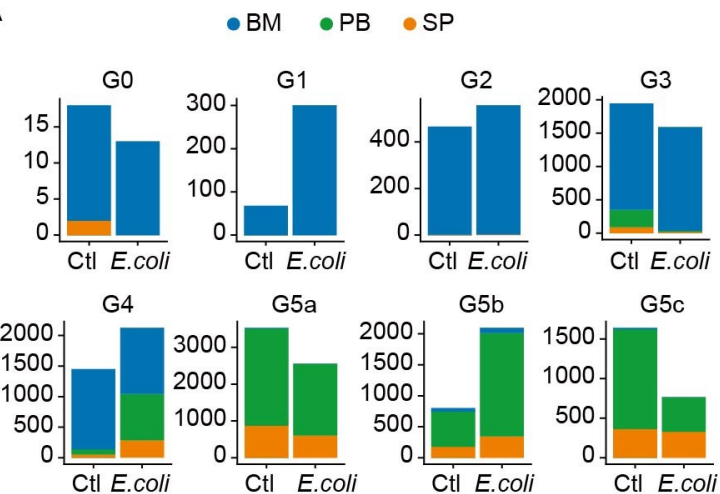

B

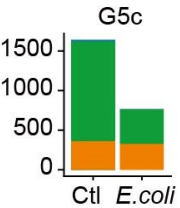

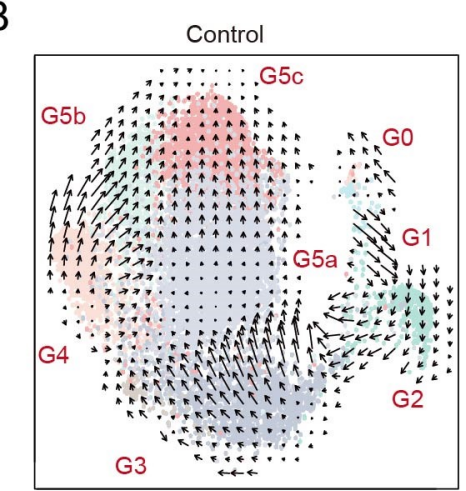

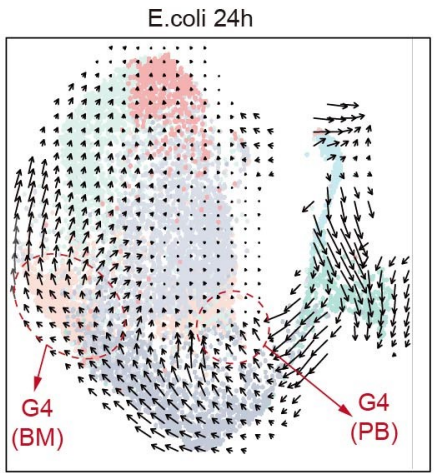

C

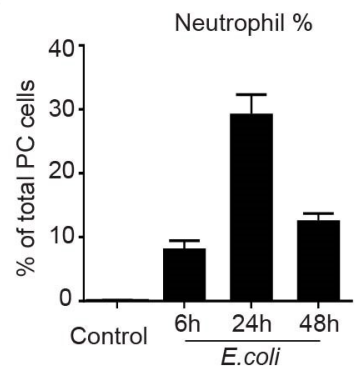

$E$

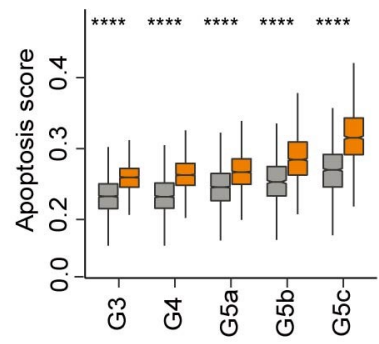

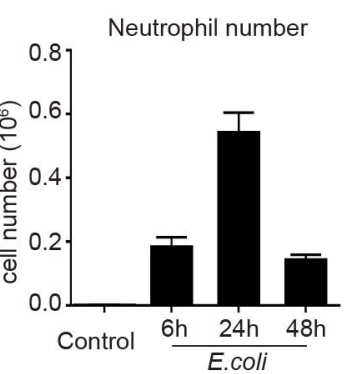

F

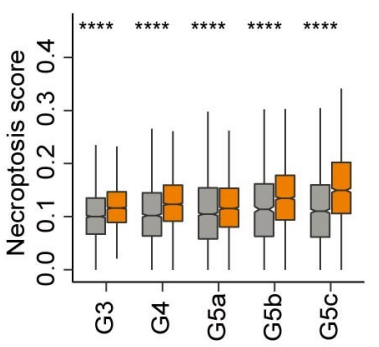

D

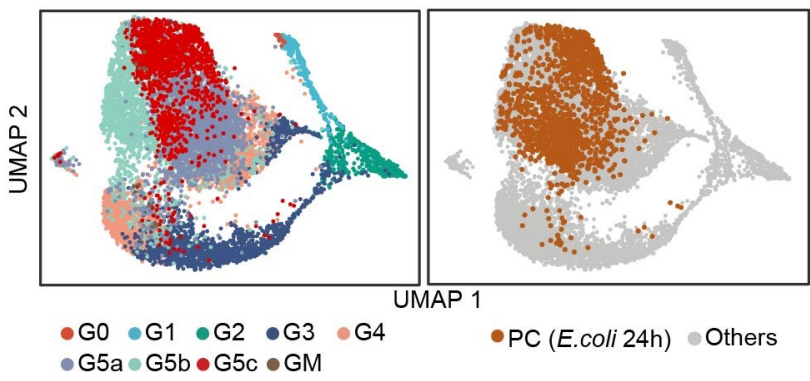

PC (E. coli $24 \mathrm{~h})$

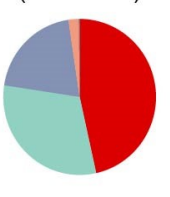

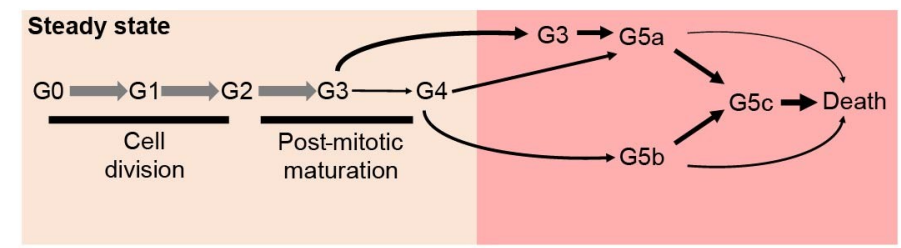

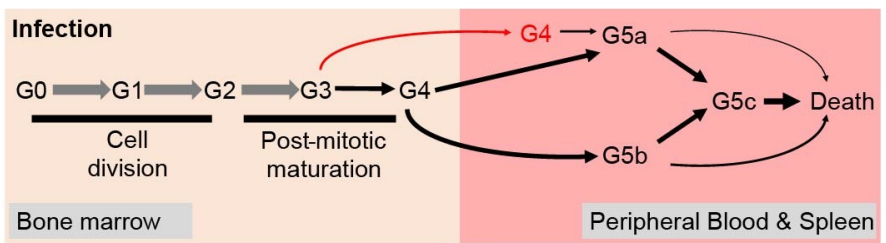


Figure 7. Bacterial infection reprograms the structure of the neutrophil population and the dynamic transition between each subpopulation.

(A) Comparisons of organ distributions of each neutrophil subpopulation before and after E. coli challenge, measured by cell number.

(B) Comparison of neutrophil dynamics (velocity field projected on the UMAP plot) before and after E. coli challenge.

(C) Neutrophil proportion and cell number in the peritoneal cavity measured at different time points after E. coli challenge. Results are the mean $( \pm \mathrm{SD})$ of three independent experiments.

(D) Left: UMAP of E. coli-challenged neutrophils from BM, PB, SP, liver (LV), and peritoneal cavity (PC) colored by cluster identity. Right: PC cells are highlighted in the UMAP plot. Proportions of each neutrophil clusters in LV were shown.

(E) Comparisons of apoptosis score and necroptosis score between control and E. coli-challenged neutrophils for G3-G5 clusters. ****, $\mathrm{P} \leq 0.0001$. Student's t-test.

(F) Dynamic transition between each subpopulation under steady-state and bacterial infection conditions. We cataloged differentiating and mature mouse neutrophils in an unbiased manner using single-cell RNA sequencing. Committed neutrophils include two proliferating subsets, two post-mitotic maturing subsets, and three functional mature subsets. Under homeostatic condition, the G5a and G5b cells in the PB arise from BM G4 and G3 cells, respectively. The transformation from G3 to G5a cells was suppressed during infection and G3 cells in infected hosts predominantly differentiated to G4 cells. 
Graphical Abstract

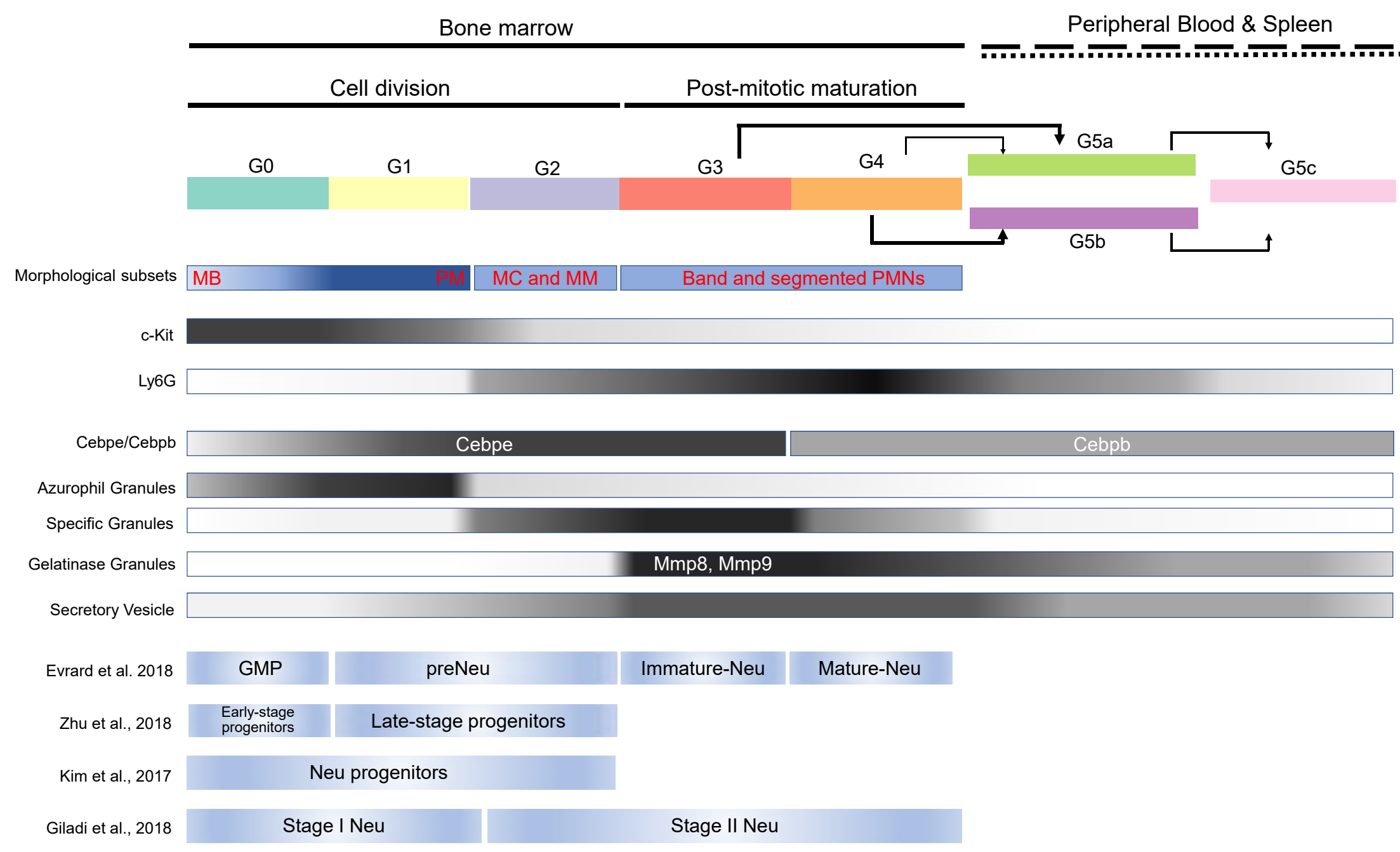




\section{KEY RESOURCES TABLE}

\section{LEAD CONTACT AND MATERIALS AVAILABILITY}

Further information and requests for resources and reagents should be directed to and will be fulfilled by the Lead Contact, Hongbo R. Luo (Hongbo.Luo@,childrens.harvard.edu).

\section{EXPERIMENTAL MODEL AND SUBJECT DETAILS}

\section{Mouse strains}

Female C57BL/6 mice were purchased from the Jackson Laboratory (Bar Harbor, ME). Eight-to-ten-week-old mice were used in all experiments. All animal experiments were conducted in accordance with the Animal Welfare Guidelines of the Children's Hospital Boston. The Children's Hospital Animal Care and Use Committee approved and monitored all procedures.

\section{Mouse peritonitis model}

Wild-type mice were intraperitoneally injected with $1 \times 10^{7}$ E. coli $\left(\mathrm{ATCC}^{\circledR} 19138^{\mathrm{TM}}\right)$ in $300 \mu \mathrm{PBS}$. At different timepoints after injection, mice were anesthetized with isoflurane, retro-orbital blood was collected, and then mice were sacrificed by euthanizing with $\mathrm{CO}_{2}$. Cells from different organs such as bone marrow, spleen, liver, and peritoneal exudate were collected as detailed below.

\section{METHOD DETAILS}

\section{Mouse neutrophil isolation}

Peripheral blood was collected by retro-orbital bleeding. 600-800 ml peripheral blood was diluted with $3 \mathrm{ml}$ HBSS containing $15 \mathrm{mM}$ EDTA. Cells were centrifuged for $10 \mathrm{~min}$ at $500 \mathrm{x}$ g. Red blood cells were lysed by resuspension in $5 \mathrm{ml} \mathrm{ACK} \mathrm{(ammonium-chloride-potassium)} \mathrm{lysis} \mathrm{buffer} \mathrm{(Thermo} \mathrm{Fisher} \mathrm{Scientific,} \mathrm{Waltham,}$ 
MA) for $5 \mathrm{~min}$ at RT. $10 \mathrm{ml}$ RPMI + 2\% FBS were added to stop lysis followed by centrifugation at $500 \mathrm{x} \mathrm{g}$ for 5 min. Cells were washed twice with $10 \mathrm{ml} \mathrm{HBSS}+2 \mathrm{mM}$ EDTA $+1 \%$ BSA before being re-suspended in 500 $\mu \mathrm{PBS}+1 \%$ BSA. For bone marrow neutrophil isolation, whole bone marrow cells were flushed from the femur, tibia, and ilia leg bones with $5 \mathrm{ml} \mathrm{HBSS}+2 \mathrm{mM}$ EDTA $+1 \%$ BSA and filtered through a $70 \mu \mathrm{m}$ cell strainer. Cells were centrifuged for $10 \mathrm{~min}$ at $500 \mathrm{x}$ g. Red blood cells were lysed with $1 \mathrm{ml}$ ACK lysis buffer for $2 \mathrm{~min}$ at room temperature (RT) and washed twice with HBSS $+2 \mathrm{mM}$ EDTA $+1 \%$ BSA and re-suspended in $200 \mu \mathrm{lBS}+1 \%$ BSA. c-kit-positive bone marrow cells were first enriched by positive selection using c-kit (CD117) microbeads (Miltenyi Biotec, Bergisch Gladbach, Germany) and further purified by FACS sorting ckit-positive cells. To isolate spleen and liver neutrophils, spleens and livers were gently disaggregated through a $70 \mu \mathrm{m}$ cell strainer with a $1 \mathrm{ml}$ syringe plunger. Whole spleen and liver cells were collected and red blood cells lysed using the same procedure as bone marrow cells. Finally, peritoneal cavity exudate cells were harvested by three successive washes with $10 \mathrm{ml} \mathrm{HBSS}+15 \mathrm{mM}$ EDTA $+1 \%$ BSA. After centrifugation, they were washed twice with the same solution and the cells re-suspended in $100 \mu \mathrm{PBS}+1 \%$ BSA. Of note, neutrophils display circadian oscillations in number and phenotype, and neutrophil aging is an intrinsically-driven bona fide circadian process (Adrover et al., 2019). Thus, all samples in this study were prepared from mice sacrificed at the same time each morning.

\section{Human sample collection}

Peripheral blood was collected from a male healthy donor aged 32 years in a heparin anticoagulant tube. $10 \mathrm{ml}$ ( $100 \%$ of blood volume) of $6 \%$ hydroxyethyl solution was added into the heparinized blood and inverted gently several times for adequate mixing. The blood was kept at RT for 30-45 min before pipetting the supernatant into a $50 \mathrm{ml}$ Falcon tube followed by centrifugation at $290 \mathrm{x}$ g for 5 mins without braking. Cells were washed twice and lysed with ACK to completely remove red blood cells. Samples were stained with Percp-cy5.5-conjugated anti-human CD33 antibody for 20 min and DAPI was added to cells prior to sorting by FACS with a FACSAria III cell sorter (BD Biosciences, Franklin Lakes, NJ). The Ethics Committee of Tianjin Blood Disease Hospital approved the study protocol, and the donor provided written informed consent for sample collection and data analysis.

\section{Single cell collection, library construction, and sequencing}

Single cell suspensions were stained for $30 \mathrm{~min}$ at $4^{\circ} \mathrm{C}$ with fluorophore-conjugated antibodies (APC/CY7conjugated anti-Gr1, FITC-conjugated-anti-CD45), filtered through $40 \mu \mathrm{m}$ cell strainers, and DAPI was added prior to sorting by FACS with a FACSAria III cell sorter (BD Biosciences). Designated cells were sorted into 
PBS containing $0.05 \%$ BSA following the $10 \mathrm{X}$ Genomics protocol. Cell preparation time before loading onto the 10X Chromium controller was $<2 \mathrm{~h}$. Cell viability and counting were evaluated with trypan blue by microscopy, and samples with viabilities $>85 \%$ were used for sequencing. Libraries were constructed using the Single Cell 3' Library Kit V2 (10X Genomics, Pleasanton, CA). Transcriptome profiles of individual cells were determined by 10X genomic-based droplet sequencing. Once prepared, indexed cDNA libraries were sequenced with paired-end reads on an Illumina NovaSeq6000 (Illumina, San Diego, CA).

\section{Bulk RNA isolation and sequencing}

BM cells were prepared as previously described. BM cells were first stained with: biotin-conjugated anti-CD4; biotin-conjugated anti-CD8a; biotin-conjugated anti-Ter119; and biotin-conjugated anti-B220/CD45R antibodies for $20 \mathrm{~min}$ and then stained with PE/cy7-conjugated streptavidin; APC-conjugated anti-c-kit; PEconjugated anti-Ly6G; FITC-conjugated anti-CD34 antibodies for $90 \mathrm{~min}$ at $4^{\circ} \mathrm{C} . \mathrm{MB}, \mathrm{PM}, \mathrm{MM}, \mathrm{MC}$, mature band, and segmented neutrophils were sorted with a Moflo cell sorter (Beckman Coulter, Brea, CA). Total RNA was extracted from those populations using the Qiagen RNeasy Mini Kit (Qiagen, Hilden, Germany). RNA quality was evaluated spectrophotometrically, and the quality was assessed with the Agilent 2100 Bioanalyzer (Agilent Technologies, Santa Clara, CA). All samples showed RNA integrity $>7.5$; RNA-seq libraries were prepared using the KAPA mRNA HyperPrep Kit (Illumina). Once prepared, indexed cDNA libraries were pooled in equimolar amounts and sequenced with paired-end reads on an Illumina HiSeq2500.

\section{Wright-Giemsa staining and examination of morphology-defined neutrophil populations}

The first recognizable cells of neutrophil lineage in the BM are myeloblasts (MBs), which are characterized by a high nuclear-to-cytoplasmic (NC) ratio and dispersed chromatin. MBs then irreversibly differentiate into promyelocytes (PMs), which are characterized by a round nucleus and azurophil granules, followed by myelocytes (MCs) characterized by a round nucleus and specific granules. Metamyelocytes (MMs) are characterized by nuclear indentations (kidney-shaped nuclei) and the emergence of secretary vesicles. Finally, MMs are divided into band cells (BCs) with a band-shaped nucleus and segmented cells (SCs, aka polymorphonuclear granulocytes) with a segmented nucleus. Cells were sorted and concentrated onto microscope slides by cytospinning. Slides were dried and stained using the Diff-Quick Stain Set (Siemens, Munich, Germany). Stained slides were rinsed under running tap water and air-dried for 10 min. Images were obtained under a microscope with a $63 x$ objective.

\section{EDU incorporation assay}


5-ethynyl-2'-deoxyuridine (EdU), a thymidine analogue, can track cells post-mitotically in the BM and PB (Fig.6e). EdU is incorporated into DNA in the S-phase of the cell cycle, and the half-life of EdU is only about $30 \mathrm{~min}$ (Cheraghali et al., 1995), so incorporation can only occur in the first 1-2 $\mathrm{h}$ after EdU intraperitoneal injection. After $1 \mathrm{~h}$ of i.p. injection with $0.5 \mathrm{mg}$ EdU, mice were injected with E. coli as above to induced peritonitis. Mice were sacrificed at designated timepoints, and BM, blood, and spleen cells were harvested followed by staining with fluorescent-conjugated antibodies: APC-conjugated anti-CD11b; APC/cy7conjugated anti-Ly6g; and PE-conjugated anti-CXCR4 antibodies. Labeled cells were fixed, permeabilized, and stained with azide dye using an EdU Proliferation Kit (BD Biosciences). Cells were further washed and analyzed using a BD FACSCanto II (BD Biosciences). Data were analyzed using FlowJo software (FloJo, BD Biosciences).

\section{Spleen cryosection preparation}

Spleens were fixed in 1\% formaldehyde (StatLab, McKinney, TX) for 4-8 h, rehydrated in 30\% sucrose solution for $72 \mathrm{~h}$, and snap frozen in OCT (Sakura Finetek, Japan). Single-cell-thick (5 $\mu \mathrm{m})$ spleen cryosections were obtained using a Leica Cryostat and the CryoJane tape transfer system (Leica Microsystems, Wetzlar, Germany). For immunofluorescent staining, slides were rehydrated in PBS for 10 min followed by rinsing in PBST (PBS + 0.1\% Tween20); blocking was performed with PBS + 10\% donkey serum for 20 min; the diluted primary rat anti-S100a8 (Thermo Fisher Scientific \#335806) and rabbit anti-Ifit1 (Abcam, Cambridge, UK \#ab236256) antibodies were added and incubated for $1 \mathrm{~h}$ at RT. After 3x washes with PBST, AlexaFluor 488conjugated donkey anti-rat antibody (Jackson ImmunoResearch, West Grove, PA \#141697) and $\mathrm{Cy}^{\mathrm{TM}} 3$ conjugated donkey anti-rabbit antibody (Jackson ImmunoResearch \#143460) were added and incubated for 30 min at RT. Slides were washed 3x with PBST and then stained with DAPI $(0.5 \mu \mathrm{M})$ for 3 min. Slides were rinsed in PBS and were covered with mounting solution (Vectashield, Vector Laboratories, Burlingame, CA).

\section{Laser scanning cytometry (LSC)}

Laser scanning cytometer (LSC) is an emerging technology that images and quantitatively analyzes cellular and subcellular criteria within tissues, re-interrogating identified cell subpopulation(s) for in situ characterization of the molecular and cellular events associated with those cells (Harnett, 2007; Kwak et al., 2015). LSC was performed with an iCys Research Imaging Cytometer four-laser system (Thorlabs, Newton, NJ). Each section was first scanned with a 10x objective using the $405 \mathrm{~nm}$ laser to generate low-resolution images of the DAPIstained nuclei and obtain a general view of the spleen. Subsequently, the sections were divided into small 
regions and scanned with a 40x dry objective lens to create high-resolution field images. Data were analyzed using iCys Cytometric Analysis Software (Thorlabs).

\section{Confocal imaging}

$20 \mu \mathrm{m}$ thick sections were prepared and stained as described above. Confocal images were obtained using the Zeiss LSM 700 Laser Scanning Confocal microscope (Carl Zeiss AG, Oberkochen, Germany). Data were analyzed using Imaris Software (Oxford Instruments, Abingdon, UK).

\section{QUANTIFICATION AND STATISTICAL ANALYSIS}

\section{scRNA-seq data processing}

The quality of sequencing reads was evaluated using FastQC and MultiQC. Cell Ranger v2.2.0 was used to align the sequencing reads (fastq) to the mm10 mouse transcriptome and quantify the expression of transcripts in each cell. This pipeline resulted in a gene expression matrix for each sample, which records the number of UMIs for each gene associated with each cell barcode. For human data, sequenced reads were aligned to the hg38 human transcriptome, then quantify the expression of transcripts in each cell using BD ${ }^{\mathrm{TM}}$ Rhapsody Whole Transcriptome Assay Analysis Pipeline. Unless otherwise stated, all downstream analyses were implemented using R v3.5.2 and the package Seurat v2.3.4 (Butler et al., 2018). Due to dissimilar data qualities, low-quality cells were filtered using sample-specific cutoffs (Table S1). The NormalizeData function was performed using default parameters to remove the differences in sequencing depth across cells.

For experiment described in Fig 1, cells from four samples were pooled and analyzed together. After rigorous quality control, we obtain 19,582 high-quality cells with average 1,241 genes per cell profiled, resulting in a total of 18,269 mouse genes detected in all cells (Fig.S1f). Clusters G1 to G5 were neutrophils at different maturation stages. G1 and G2 were early-stage neutrophils with higher expression of Elane, Mpo, Fcnb, and Camp (Fig.1d-e). Neutrophils are terminally differentiated. The transition from a proliferative cell to terminal differentiation was accompanied by a dramatic change in expression of important cell-cycle regulatory proteins, so we next performed a single-cell resolution analysis of cell cycle activation during neutrophil differentiation based on the expression of G1/S and G2/M phase-specific genes (Kowalczyk et al., 2015; Tirosh et al., 2016b) (Fig.1i). Cells in the G0 to G2 stages underwent active proliferation, while cell division stopped abruptly thereafter. CDC28 Protein Kinase Regulatory Subunit 2 (CKS2), Mki67, and Cdc20 were all strongly downregulated at the mRNA level. 
For experiment described in Fig 4, After excluding low-quality cells, a total of 25,897 cells, including 4421 cells from the BM (eBM_Gr1), 6232 cells from the PB (ePB_Gr1), 5989 cells from the SP (eSP_Gr1), 4435 cells from the liver (eLV_Gr1), and 4169 cells from the peritoneal cavity (ePC_Gr1) were available for analysis (Fig.S4b-c).

\section{Batch correction}

There was substantial variability between cells obtained from different samples, likely reflecting a combination biological and technical differences. In this case, the batch had little effect on partitioning cell types and thus cell clustering into neutrophils, B cells, T cells, monocytes, dendritic cells, erythrocytes, and progenitors. However, when clustering neutrophils alone, cells clustered first by sample rather than by biological clusters. Therefore, the ScaleData function was used to eliminate cell-cell variation in gene expression driven by batch and mitochondrial gene expression. Importantly, the batch-corrected data were only used for principal component analysis (PCA) and all steps relying on PCA (e.g., clustering, UMAP visualization); all other analyses (e.g., differential expression analysis) were based on the normalized data without batch correction.

\section{Dimension reduction}

Dimension reduction was performed at three stages of the analysis: the selection of variable genes, PCA, and uniform manifold approximation and projection (UMAP) (Becht et al., 2018b). The FindVariableGenes function $(y . c u t o f f=1$ for control total cells; y.cutoff $=1.2$ for control neutrophils; y.cutoff $=0.7$ for $E$. colichallenged total cells) was applied to select highly variable genes covering most biological information contained in the whole transcriptome. Then, the variable genes were used for PCA implemented with the RunPCA function. Next, we selected PCs 1-20 (for total cells) or 1-15 (for neutrophils) as input to perform the RunUMAP function to obtain bidimensional coordinates for each cell.

\section{Unsupervised clustering}

We performed the FindClusters function (resolution 0.3, 0.6, and 0.2 for control total cell, neutrophils, and $E$. coli-challenged total cells, respectively) to cluster cells using the Louvain algorithm based on the same PCs as RunUMAP function.

\section{Identification of differentially expressed genes}


We used the FindMarkers or FindAllMarkers function (test.use $=$ ' 't'”, logfc.threshold $=\log (2)$ ) based on normalized data to identify differentially expressed genes (DEGs). P-value adjustment was performed using Bonferroni correction based on the total number of genes in the dataset. DEGs with adjusted p-values $>0.05$ were filtered out. Gene ontology (GO) analysis was performed by using the R package clusterProfiler (Yu et al., 2012). In experiment described in Fig S5, we conducted differential gene expression analysis in each neutrophil subpopulation using the non-parametric Wilcoxon rank sum test and identified DEGs with an average expression fold-change $>2$.

\section{Developmental trajectory inference}

Pseudotime was generated with Monocle v2 (Qiu et al., 2017b) to infer the potential lineage differentiation trajectory. The newCellDataSet function (lowerDetectionLimit $=0.5$, expressionFamily $=$ negbinomial.size) was used to build the object based on the above highly variable genes identified by Seurat v2.3.4.

\section{Bulk RNA-seq analysis}

The quality of sequencing reads was evaluated using FastQC and MultiQC. Adaptor sequences and low-quality score bases were trimmed using trimmomatic/0.36. The resulting reads were then mapped to the mouse reference sequence (GRCm38/mm10, Ensemble release 81) and counted using STAR2.5.2b alignment software. Gene differential expression analysis was performed using the R package EdgeR.

\section{Scoring of biological processes}

Individual cells were scored for their expression of gene signatures representing certain biological functions. For all signatures except neutrophil aging, functional scores were defined as the average normalized expression of corresponding genes. Aging score was defined as the weighted average of Z-scores of age-related genes, where the Z-scores were calculated by scaling the normalized expression of a gene across all cells. Gene weights were set to either 1 or -1 to reflect positive or negative relationships. The neutrophil maturation signature was derived by identifying the top 50 DEGs with highest fold changes and adjusted p-values $<0.05$ between mature cluster (G4) with immature clusters (G0-G3). Granule signatures were from (Cowland and Borregaard, 2016b). Other functional signatures were derived from the GO database (Consortium, 2018), with the full gene list provided in Table S4. For instance, to access the phagocytosis function at the transcript level, we determined a "phagocytosis score" by calculating the average expression of genes in the GO term "phagocytosis, engulfment" (GO:0006911). Apoptosis score was measured by the upregulation of the integrated proapoptotic pathway and downregulation of pro-survival gene expression (Fig 3b). To further dissect apoptotic 
heterogeneity in G5 populations independently of transcriptome-based sub-clustering, we fit a two-component Gaussian mixture model to the apoptotic score of all G5 cells using the R package mixtools version 1.1.0 (Benaglia et al., 2009). We then chose the distribution with the higher mean as the apoptotic group and assigned each cell to one of the two groups based on its posterior (Fig 3c).

Age-related genes were summarized from the previous literature (Fig 2i). Related to function, aged neutrophils express less adhesion molecule L-selectin (Cd62L, Sell) and more CD11b ( $\alpha \mathrm{M}$, Itgam), lymphocyte functionassociated antigen-1 (CD11a/ß2), CD49d (integrin $\alpha 4$, Itga4), TLR4, ICAM-1, CD44, and CD11c (Itgax) (Casanova-Acebes et al., 2013; Uhl et al., 2016; Zhang et al., 2015). Additionally, aged neutrophils express more surface CXCR4 and less CXCR2, which regulates their release from and return to the BM (Eash et al., 2010; Eash et al., 2009; Martin et al., 2003; Nagase et al., 2002; Zhang et al., 2015). CXCR4 may also play a role in clearing aged, senescent neutrophils, particularly at BM sites. Anti-CXCR4 antibodies or CXCR4 antagonists impede neutrophil homing to the BM (Martin et al., 2003; Suratt et al., 2004). Finally, aged neutrophils exhibit increased expression of CD24, a GPI-linked glycoprotein which induces apoptosis when crosslinked (Parlato et al., 2014) and reduced expression of CD47, the "don't eat me" signal that inhibits efferocytosis, a process leading to clearance of dead neutrophils (Jaiswal et al., 2009; Zhang et al., 2015). ROS-mediated pathogen killing is a major host defense mechanism. In neutrophils, ROS are mainly produced by the phagocytic NADPH oxidase (aka the NOX2 complex). During cell activation, cytosolic components of the NADPH oxidase NCF2 (p67phox), Rac1 and/or Rac2, NCF4 (p40-phox), and NCF1 (p47phox) are recruited to the membrane to form a complex with membrane proteins CYBA (p22-phox) and CYBB (gp91 or cytochrome-b 558 subunit beta) (Babior et al., 2002; Dahlgren and Karlsson, 1999; Henderson and Chappel, 1996; Heyworth et al., 2003; Luo and Loison, 2008; Nauseef and Borregaard, 2014; Segal et al., 2000; Subramanian and Luo, 2009). We evaluated the "NADPH oxidase score" based on the expression of the seven NADPH oxidase-related genes (Fig S3d)

\section{Comparison of scRNA-seq-defined populations with morphology-defined neutrophil subpopulations}

To benchmark single-cell transcriptomic neutrophil classification against existing morphological classification schemes, we deconvoluted bulk RNA-seq profiles based on expression of scRNA-seq-identified group-specific signatures. This approach was similar to other existing deconvolution methods like CIBERSORT (Newman et al., 2015), but we used a linear regression model with the constraint of non-negative coefficients (i.e., nonnegative least-squares problem) instead of the linear support vector regression in CIBERSORT. Although we manually chose 20 genes with highest fold-changes as signatures for each single cell group, we noted that the deconvolution in our case was robust to the choice of signatures. The regression model was built using $\mathrm{R}$ package nnls version 1.4 (Katharine M. Mullen, 2012). Bulk profiles were quantile normalized. 
At different morphology-defined neutrophil differentiation stages, neutrophils produce different granules containing distinct enzymes and antimicrobial compounds. Thus, we also examined the expression of various granule genes in differentiating neutrophils. Genes related to primary (azurophilic) granules such as Mpo started to be expressed in some G0 cells, peaked in G1 cells, and then rapidly decreased in G2 cells (Fig.2a-b). MPOnegative granules can be divided into granules containing lactoferrin (LTF) but no gelatinase (MMP9), granules that contain both, and granules that contain gelatinase but no lactoferrin (Kjeldsen et al., 1993). We found sequential production of these granules in maturing neutrophils, with lactoferrin-containing granules emerging in G2 cells, lactoferrin and gelatinase-containing granules emerging in G3 cells, and gelatinase-containing granules (Ltf low) emerging in G4 cells (Fig.2a-c). Of the proteins that localize exclusively to secretory vesicles such as Frp1 and Vamp2, their cognate mRNA profiles peaked in G4 cells in the BM and continued to be expressed in PB neutrophils.

\section{SCENIC analysis}

SCENIC is a computational tool that infers regulatory modules or regulons by analyzing co-expression of transcription factors (TFs) and their putative target genes characterized by enrichment of corresponding TFbinding motifs in their regulatory regions (Aibar et al., 2017). Regulatory network analysis was performed on all control and E.coli-challenged samples using Python package pySCENIC version 0.9.11 (Aibar et al., 2017) with default parameters. We scaled the network inference step by first inferring regulons on a 6000-cell subset, then calculated AUCell scores for all 32,888 cells included in this analysis. Specifically, we randomly sampled 300 cells from each neutrophil population in each condition and 1200 non-neutrophil cells as the training set for network inference. Output co-expression modules were trimmed with cisTarget databases (mm10_refseqr80_500bp_up_and_100bp_down_tss, mm9-tss-centered-10kb-7species, and mm9-500bp-upstream-10species). The identified 413 regulons were then scored to determine their activities in each cell. K-means clustering was performed on the first 20 principle components (PCs) of regulon activity matrix with cluster number $\mathrm{k}=7$.

\section{Differential activity analysis of SCENIC regulons}

To assess the effect of biological conditions on regulon activity, we applied a generalized linear model (GLM) as reported in (Lambrechts et al., 2018). We compared the AUCell score of each regulon with different baseline clusters corresponding to different biological questions like neutrophil cluster transition and infection response. GLM results were further filtered by p-values and visualized using R package ComplexHeatmap (Gu et al., 2016). 


\section{RNA velocity analysis}

Cell RNA velocity analysis was performed using Velocyto program (La Manno et al., 2018a). This approach uses the relative proportion of unspliced and spliced mRNA abundance as an indicator of the future cell state (La Manno et al., 2018b). The calculated RNA velocity is a vector that predicts individual cell transition, with the direction and speed of each transition assessed based on the amplitude and direction of individual cell velocity arrows on the UMAP plot. Accordingly, the hierarchical relationship between two cell populations can be inferred by the directional flow in the RNA velocity vector field. Annotation of spliced and unspliced reads was first performed using velocyto.py command-line tools. Then, downstream analysis was performed using the velocyto.R pipeline. We retained the genes expressed in at least one cell population. In total, 4815 genes were used for the analysis. RNA velocities of each cell were estimated using the gene.relative.velocity.estimates function. Finally, the velocity field was projected onto the existing UMAP space.

\section{Cell label transfer}

Total cells were partitioned into distinct cell types annotated by the expression of known marker genes. Neutrophils in their steady state were partitioned into eight clusters based on gene expression profiles annotated according to their development order. E. coli-challenged neutrophils were annotated using a well-accepted method (Stuart et al., 2019). We first identified pairwise correspondences (aka anchors) between single cells across datasets (before and after E. coli challenge) to quantify the batch effect. Each cell in the E. colichallenged dataset was then annotated based on the transcriptomic similarity between this cell and cells in the reference dataset. Specifically, cells would receive corresponding labels with the highest similarity scores, whereas cells with the highest similarity score lower than 0.5 were defined as "unassigned" and were not represented in the reference. In this way, each neutrophil from the new stimulated dataset was assigned a cluster name, and neutrophils sharing similar transcriptomic profiles would be placed into the same cluster. Hence, each cell in the bacterial infection state was assigned to one of the nine cluster labels. This transfer procedure was implemented using the FindTransferAnchors $(\operatorname{dims}=1: 15)$ and TransferData $(\operatorname{dims}=1: 15)$ functions in Seurat v3.0.2 (Stuart et al., 2019) with the combination of top 100 DEGs of each cluster.

\section{DATA AND CODE AVAILABILITY}

All sequencing data have been deposited at NCBI GEO depository and are accessible with the accession number GSEXXX. 


\section{Supplementary materials}

Single-cell transcriptome profiling reveals neutrophil heterogeneity and orchestrated maturation during homeostasis and bacterial infection

Xie et al. 


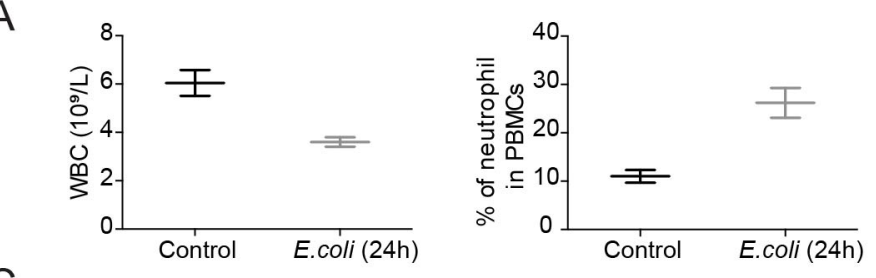

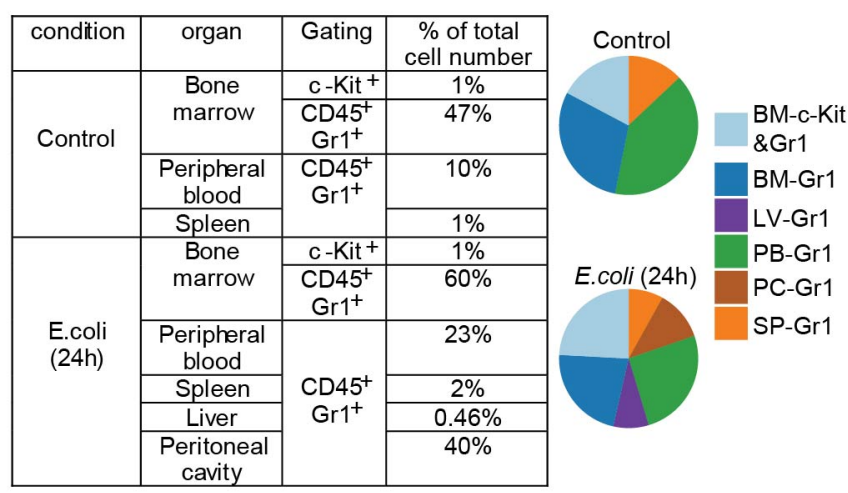
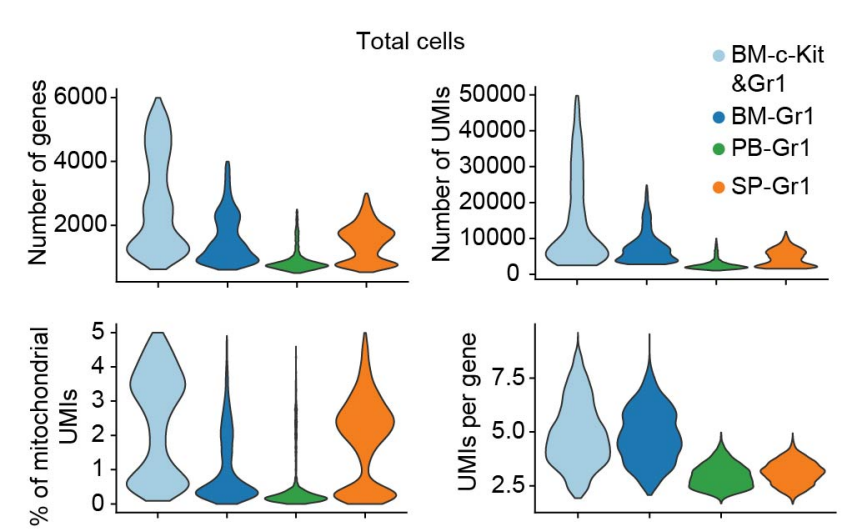

$\mathrm{H}$
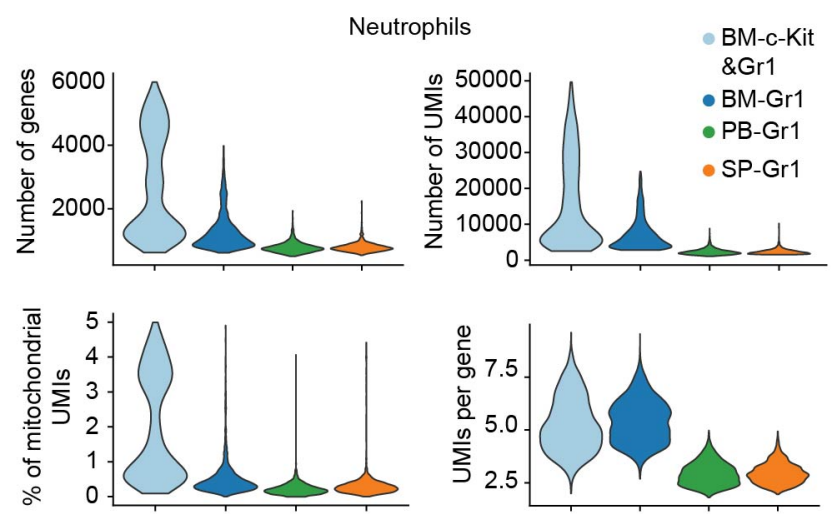

- Ctrl_BM_Ly6g (Amit Lab) - Ctrl_BM_Gr1
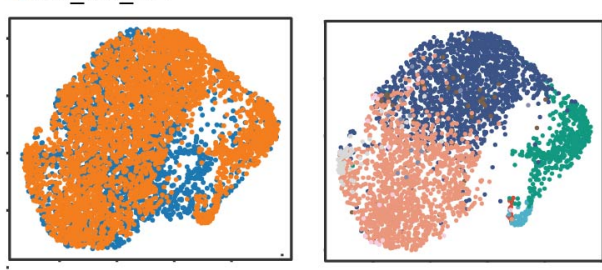

Proportion

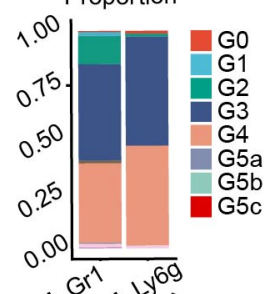

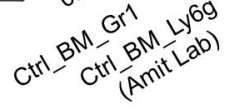

B
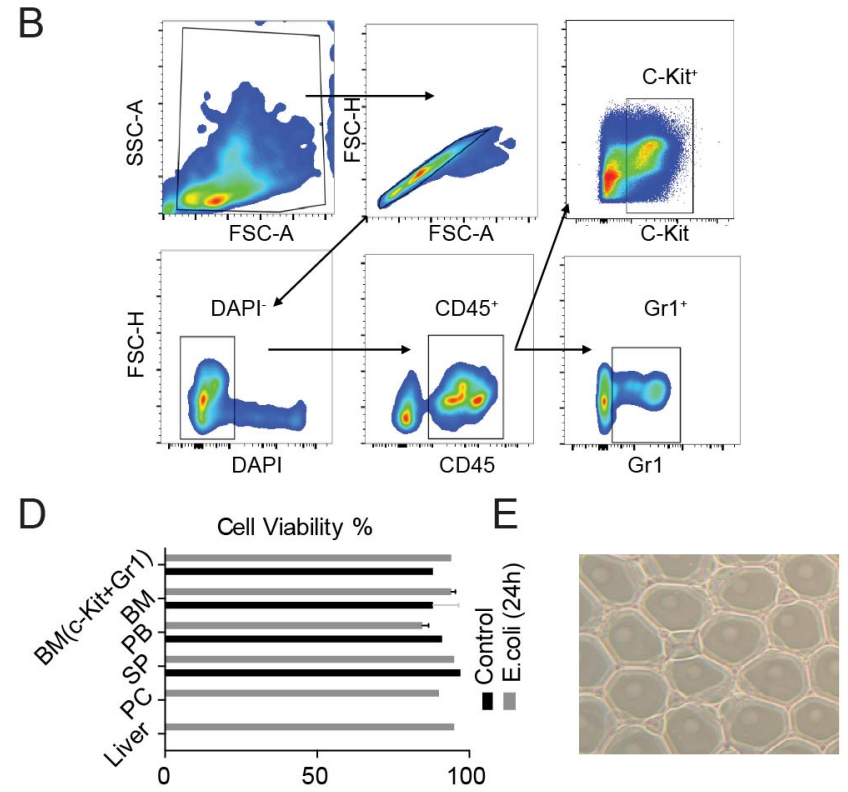

G
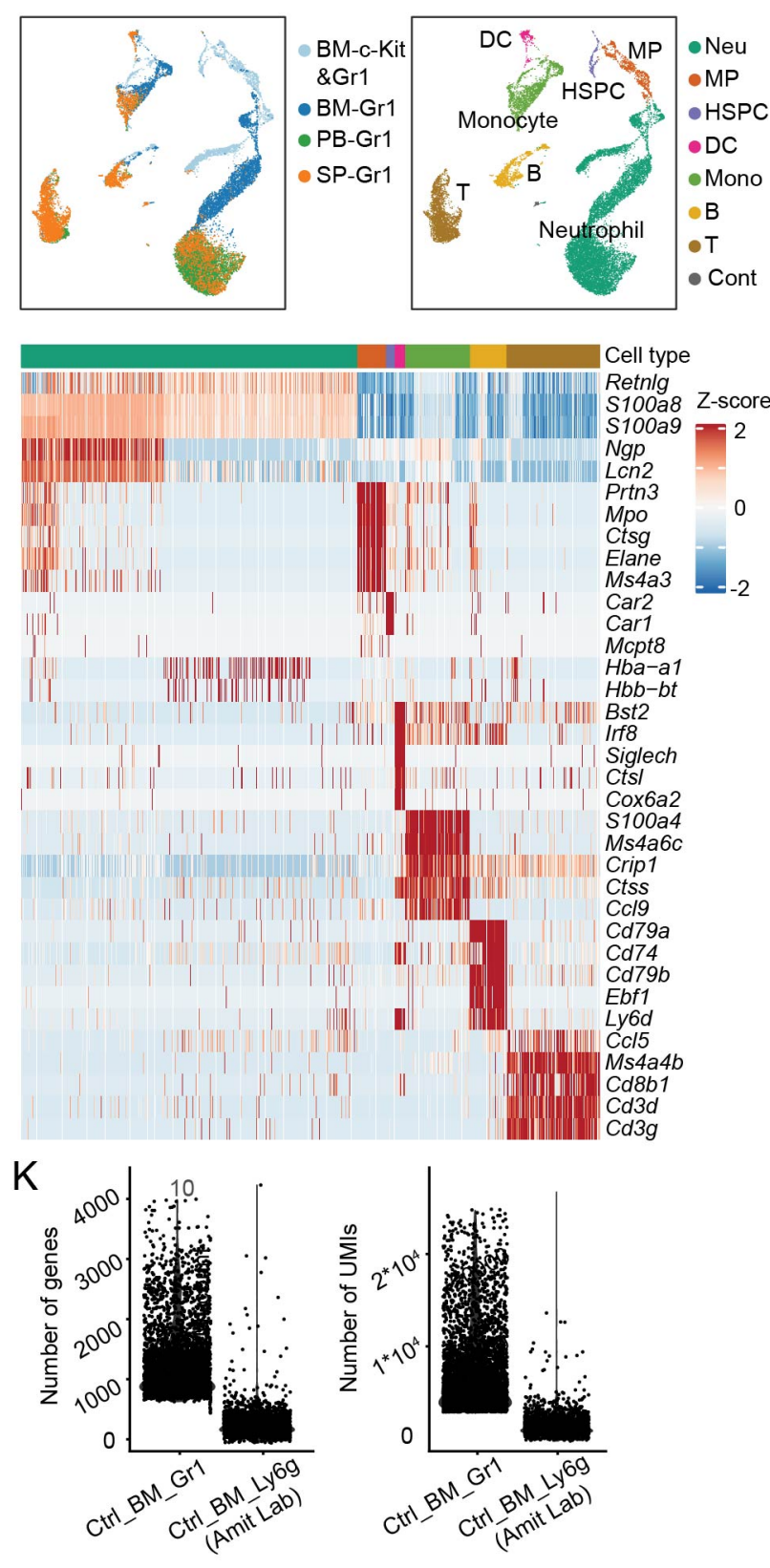
Figure S1. Sample preparation, quality controls, and related parameters and results related to scRNAseq analysis.

(A) Number of white blood cells and the proportion of neutrophils in mice before and after E. coli challenge evaluated by a hematology analyzer (Mindray BC-5000 Vet). Results are the mean \pm SD of three independent experiments.

(B) Fluorescence-activated cell sorting (FACS) strategy for scRNA-seq sample preparation.

(C) Summary of sample information. Organ distributions of neutrophils before and after E. coli challenge are shown on the right.

(D) Cell viability percentages immediately before cells were loaded into the 10X Chromium Controller.

(E) Representative GEM formation after the 10X Chromium Controller under the microscope.

(F) Violin plots of the number of genes, number of UMIs, mitochondria count percentage, and UMI per gene of all QC-passed cells in different organs.

(G) Uniform manifold approximation and projection (UMAP) of 19,582 cells from the bone marrow (BM), peripheral blood (PB), and spleen (SP) colored by sample origin and cell type, respectively. Expression of unique genes specifically distinguished each cluster and associated them with neutrophils (Neu) (S100a8 and S100a9), myeloid progenitors (MP) (Cd34, Kit, Mpo and Elane), hematopoietic stem progenitor cells (HSPC, not including MP) (Cd34, Kit, Mpo and Elane'), monocytes (Mono) (S100a4 and Ccl9/MIP-1 $\gamma$ ), B cells (Cd79a and Cd79b), T cells (CD3d and Ccl5), and dendritic cells (DC) (Siglech), respectively. Cont: contaminated cells.

(H) As in (F) but using only neutrophils in different organs.

(I) Heatmap showing the five highest differentially expressed genes (DEGs) per cell type for all QC-passed cells.

(J) Comparison of $\mathrm{Gr}^{+} \mathrm{BM}$ neutrophil populations in our data with $\mathrm{Ly}_{6} \mathrm{~g}^{+} \mathrm{BM}$ neutrophil populations in Dr. Ido Amit's data. Cluster labels are transferred from our data to Dr. Ido Amit's data (Giladi et al., 2018)(Methods). Left: UMAPs of $3591 \mathrm{Gr}^{+}$neutrophils and $2304 \mathrm{Ly} \mathrm{g}^{+}$neutrophils colored by data set or cluster identity. Right: Neutrophil compositions in our data and Dr. Ido Amit's data.

(K) Violin plots of the number of genes and number of UMIs of our $G r 1^{+}$neutrophils and Dr. Ido Amit's $\mathrm{Ly} 6 \mathrm{~g}^{+}$ neutrophils. 
A

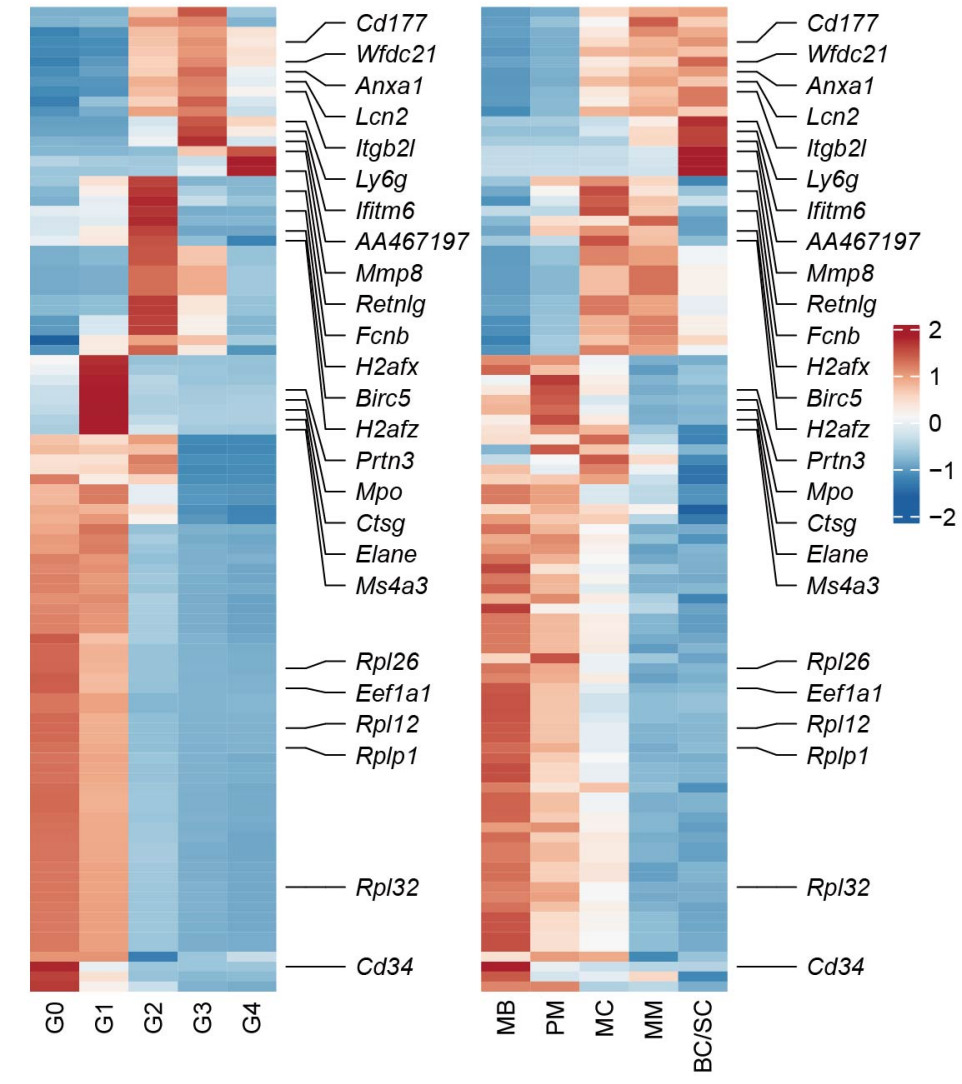

B

Granule-related genes

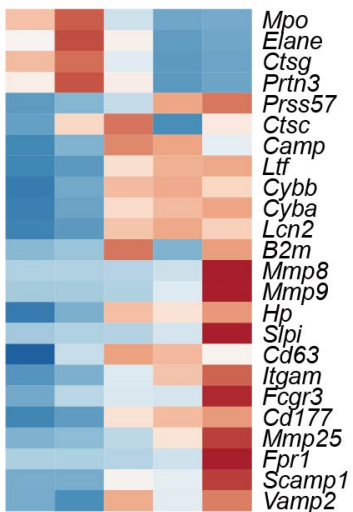

$\sum \sum \sum \sum \underset{\substack{0 \\ 0}}{m}$

F

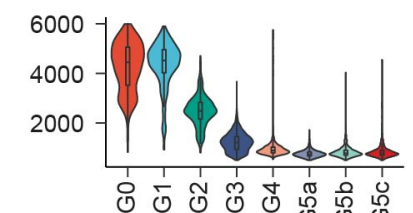

C

Cell-cycle related genes

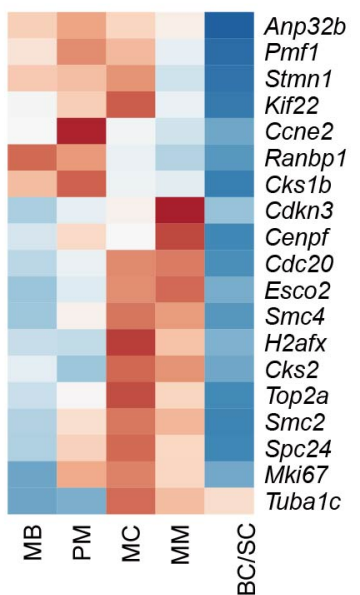

E

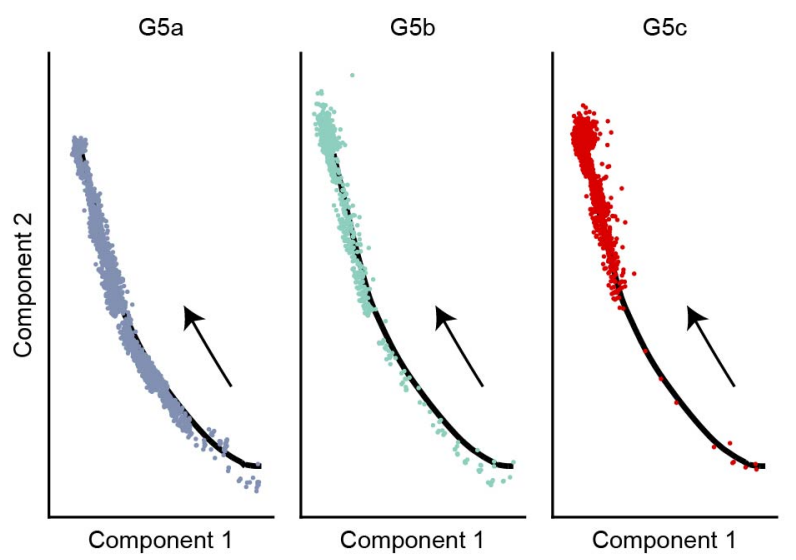

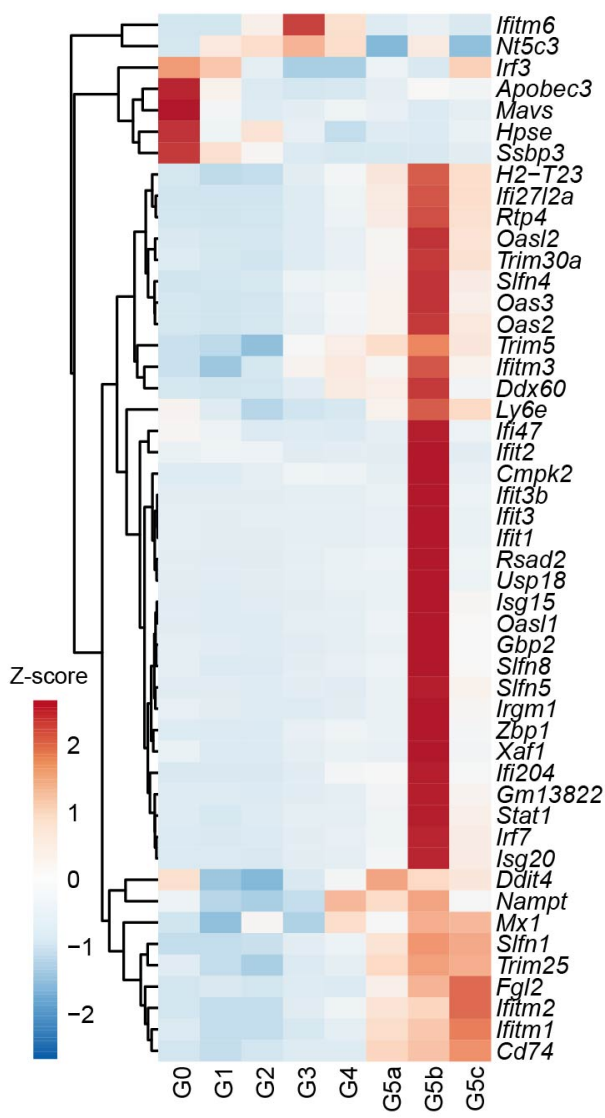

G5c 


\section{Figure S2.}

(A-C) scRNA-seq defined differentiating neutrophil populations correlated with classical morphologydefined neutrophil subpopulations.

(A) Heatmaps showing row-scaled expression of the 20 highest DEGs per cluster across averaged single-cell groups (left) and morphological groups (right). Only genes detected in both scRNA-seq data and bulk RNA-seq data are visualized. Representative genes are indicated.

(B-C) Heatmaps showing row-scaled expression of granule-related genes (B) and cell-cycle related genes (C) for morphological groups.

(D) Heatmap showing row-scaled expression of 47 interferon-stimulated genes for each averaged cluster.

(E) Monocle trajectories of neutrophil population G5a, G5b, and G5c. Each dot represents a single cell.

(F) Violin plots of the number of genes, number of UMIs, mitochondria count percentage, and UMI per gene of neutrophils in each cluster. 
A

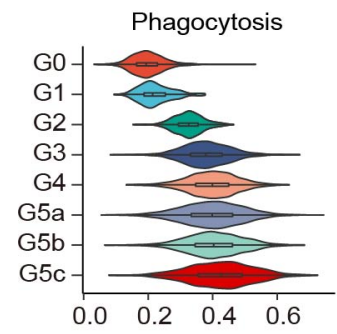

E

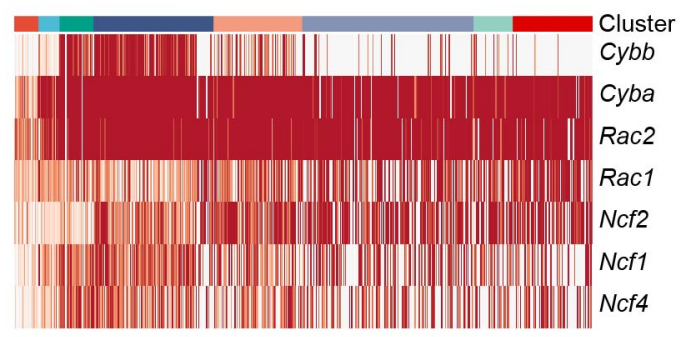

Glycolysis
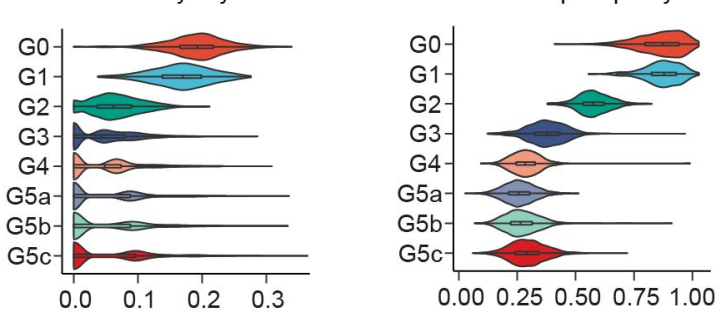

C

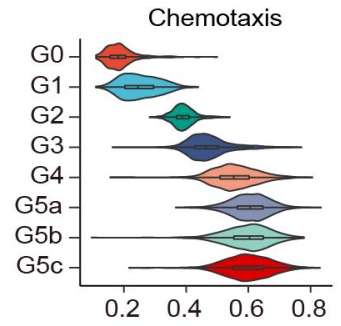

Neutrophil activation

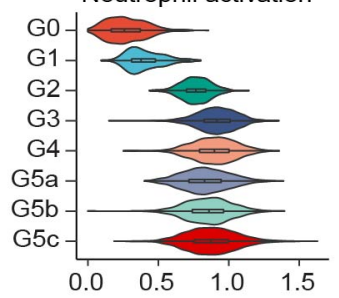

F Mitochondria-mediated

D

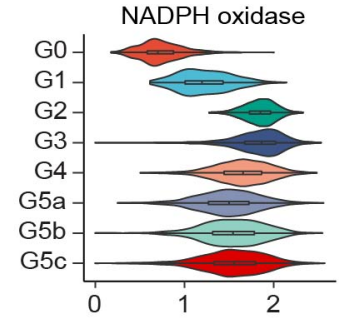
ROS production

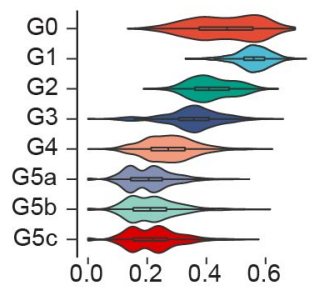

Tricarboxylic acid cycle
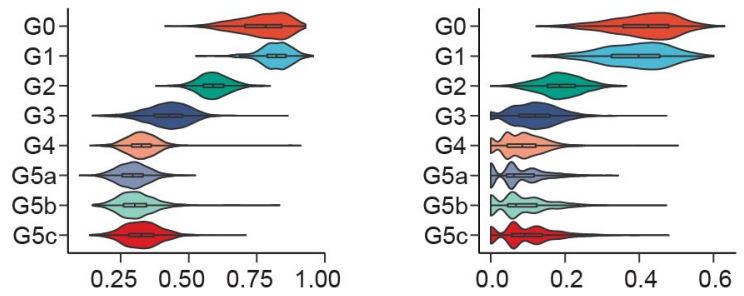

I

Glycolysis

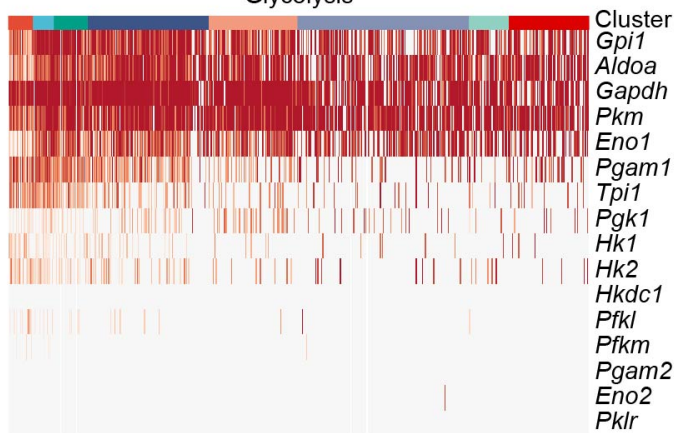

Glucose transport

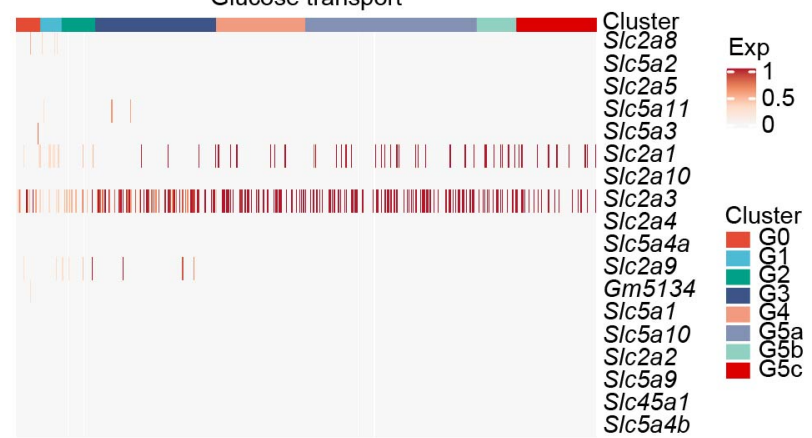

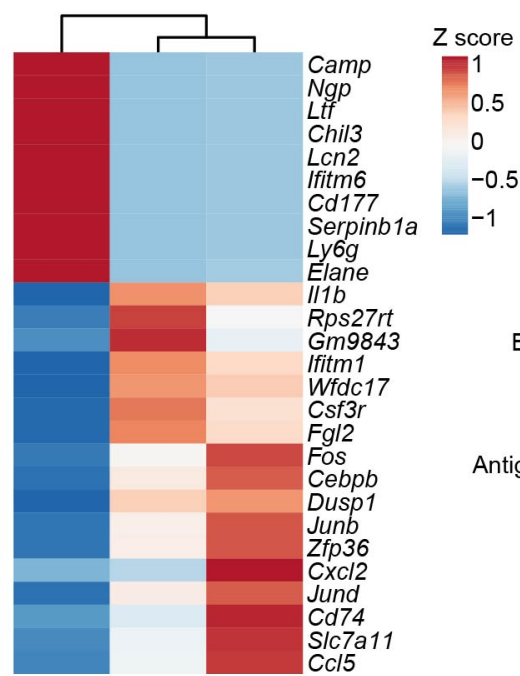

0.5

$-0.5$$$
-1
$$

African trypanosomiasis

Osteoclast differentiation $B$ cell receptor signaling pathway

Ribosome

Antigen processing and presentation IL-17 signaling pathway

TNF signaling pathway

Th17 cell differentiation Leishmaniasis

Th1 and Th2 cell differentiation Yersinia infection

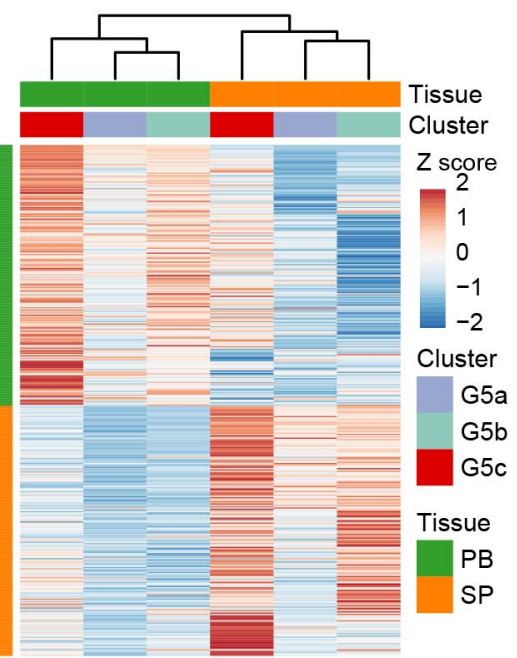




\section{Figure S3.}

\section{(A-I) Characterization of neutrophil subpopulations.}

(A-D) Violin plot of phagocytosis score (GO:0006911), chemotaxis score (GO:0030593), neutrophil activation score (GO:0042119), and NADPH oxidase score for each cluster.

(E) Heatmap showing relative expression of seven genes of the NADPH oxidase complex for all neutrophils.

(F) As in (A-D) but displaying mitochondria-mediated ROS production score (reactive oxygen species biosynthetic process, GO:1903409) for each cluster.

(G) Violin plots of metabolic scores for each cluster. Glycolysis (Reactome Pathway Database \#R-MMU70171); Oxidative phosphorylation (GO:000619); Electron transport chain (GO:0022900); Tricarboxylic acid cycle (GO:0006099).

(H-I) Heatmaps showing relative expression of glycolysis-related genes and glucose transport-related genes.

\section{(J-K) Organ-specific transcriptome features.}

(J) Heatmap showing row-scaled expression of the ten highest DEGs per organ for each averaged organ profile.

(K) As in (J) but for each G5 subpopulation between PB and SP. KEGG analysis of DEGs for each G5 in these two organs. Left: selected KEGG terms with Benjamini-Hochberg-corrected P-values $<0.05$ are shown. 
A

B

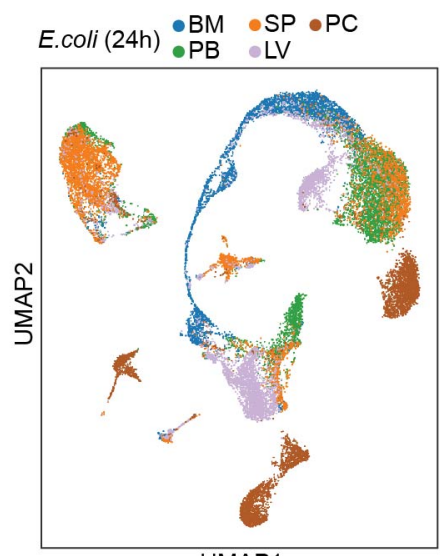

UMAP1
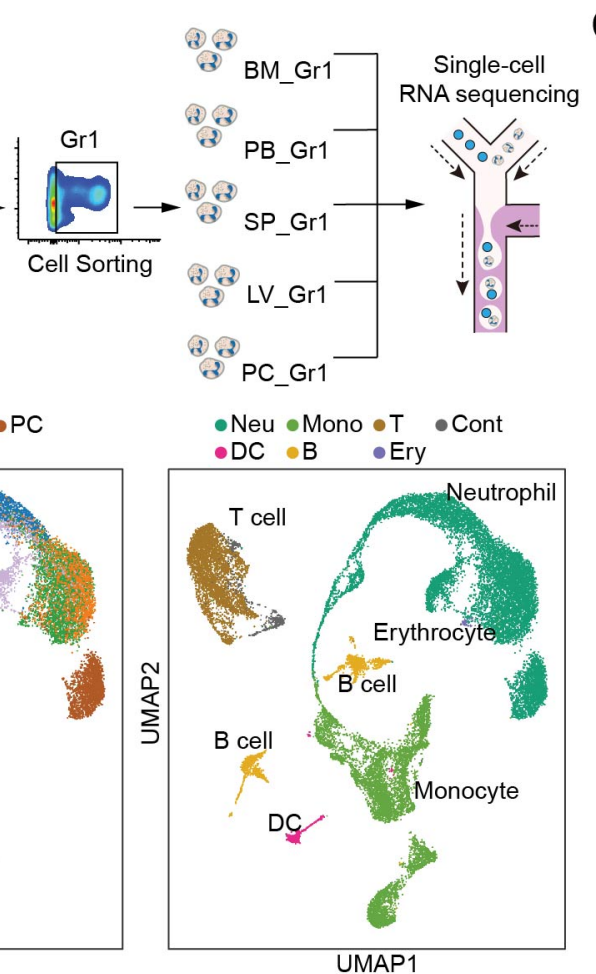

C

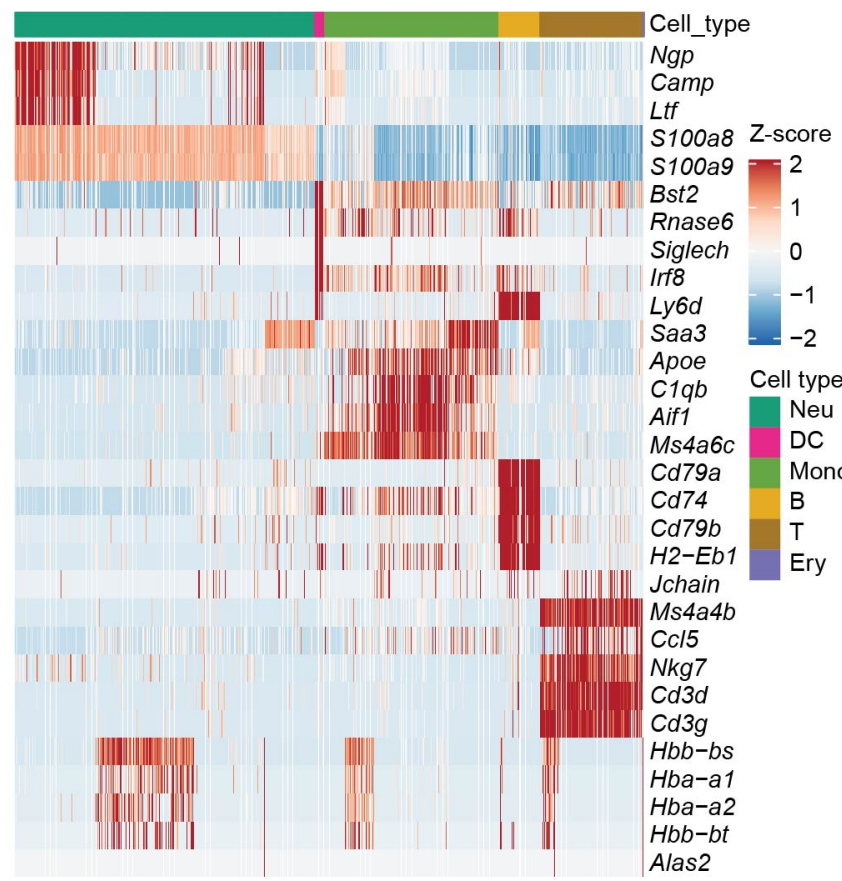

E

Total cells

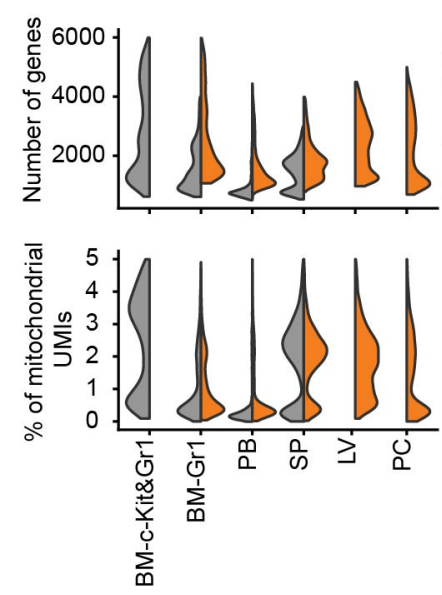

Ecoli
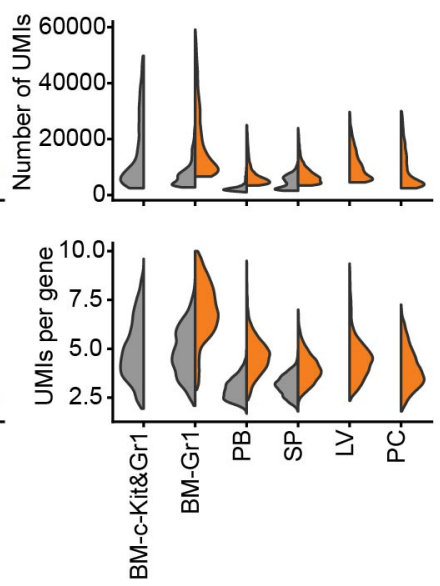

G
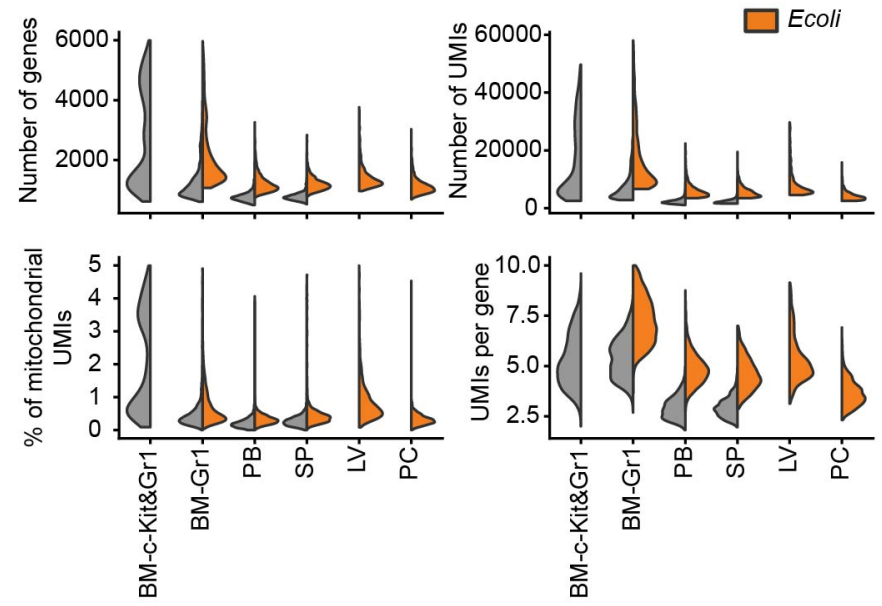

Ecoli

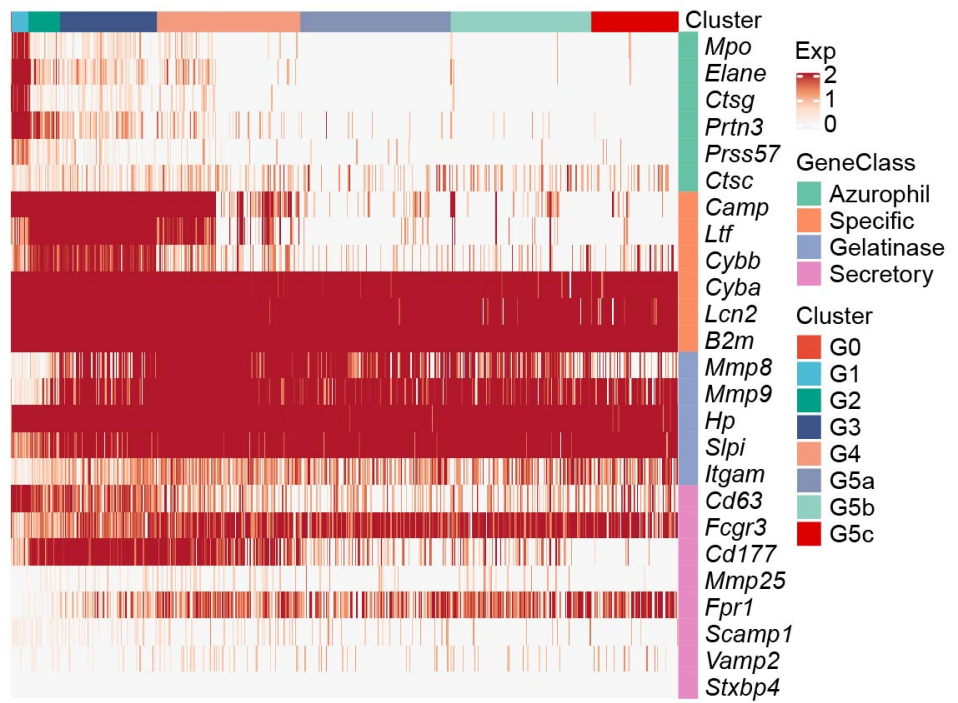


Figure S4. Single cell RNA-seq analysis of neutrophils in E. coli-challenged mice.

(A) Experimental scheme of the sample collection process after E. coli challenge.

(B) UMAPs of all 24,943 cells from BM, PB, and SP from E. coli-challenged mice colored by sample origin and cell type, respectively.

(C) Heatmap showing row-scaled expression of the five highest DEGs for all QC-passed cells colored by cell type.

(D) Comparisons of the number of genes, number of UMIs, mitochondria count percentage, and UMI per gene of all QC-passed cells in each organ before and after E. coli challenge.

(E) As in (D) but only of all neutrophils in each organ.

(F-G) Heatmaps showing expression of 7 genes of the NADPH oxidase complex (F) and neutrophil granulerelated genes $(\mathrm{G})$ for all neutrophils. 


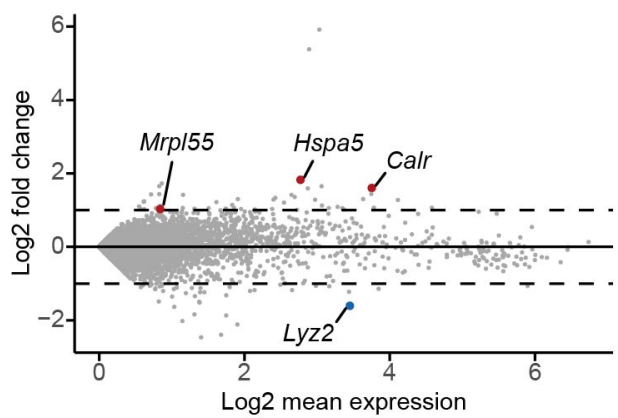

G3 -Up: 19 •Down: 25 •NS

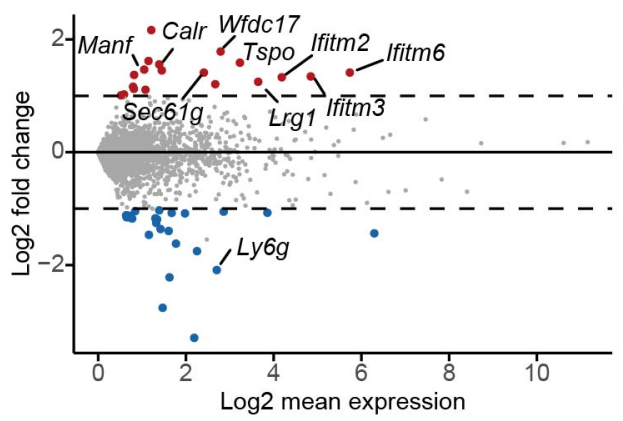

G5b

-Up: 24 •Down: 24 -NS

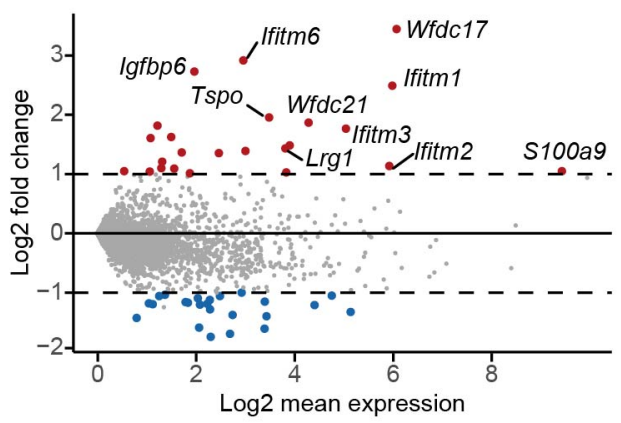

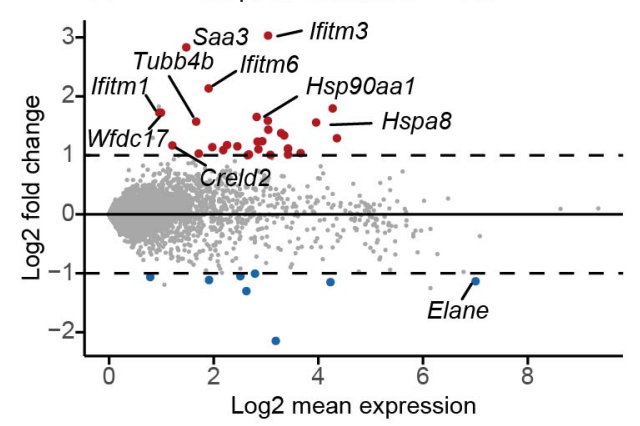

G4 •Up: 19 •Down: 11 •NS

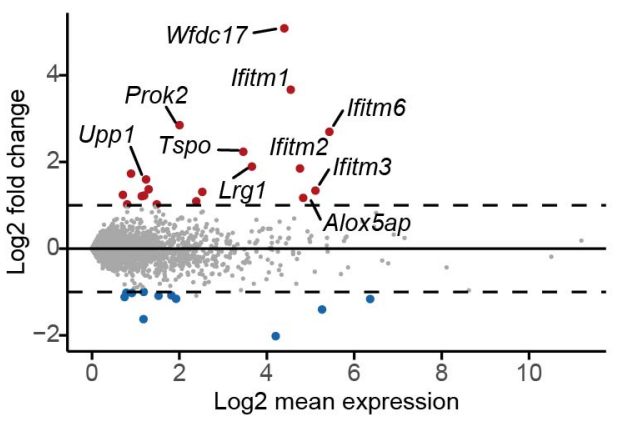

G5c -Up: 20 - Down: 32 -NS

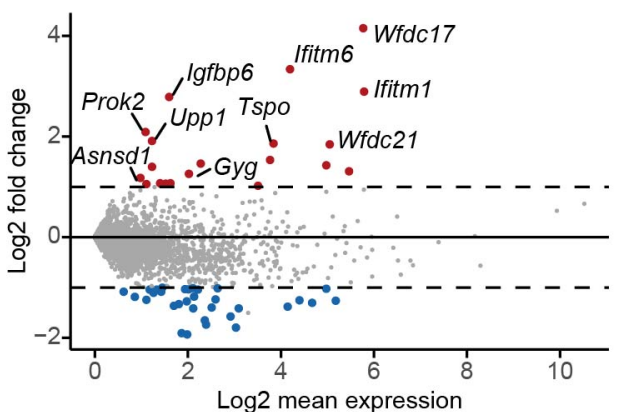

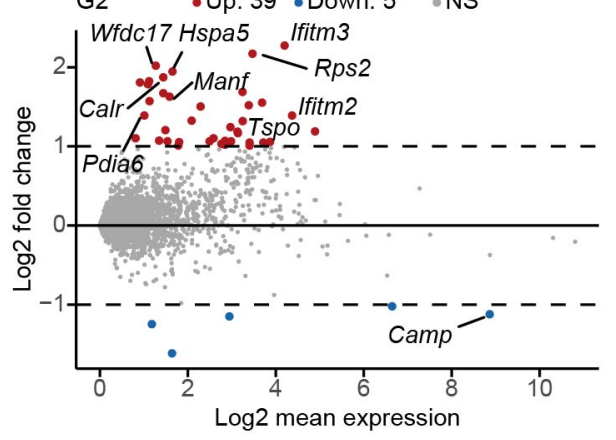

G5a •Up: 21 •Down: 24 •NS

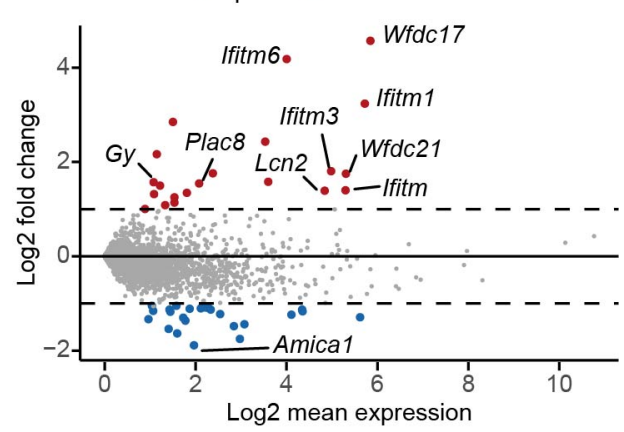

B

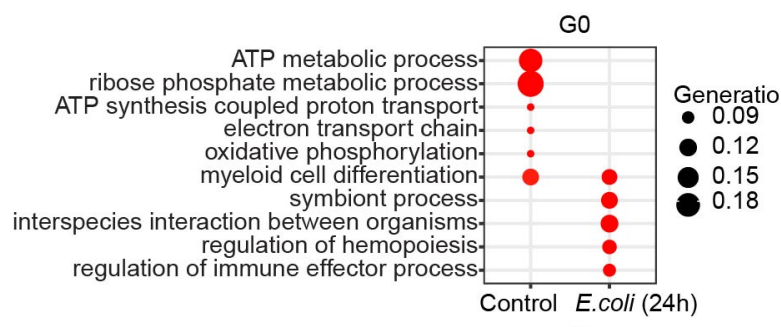

dosage compensation by inactivation of $X$ chromosomedosage compensation regulation of gene expression, epigenetic homeostasis of number of cells cytoplasmic translation-
cyte lipoprotin particle stimulus protein folding. myeloid cell homeostasis cell killing.

Control E.coli (24h)

G2

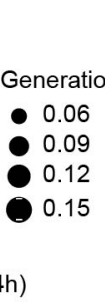

G4

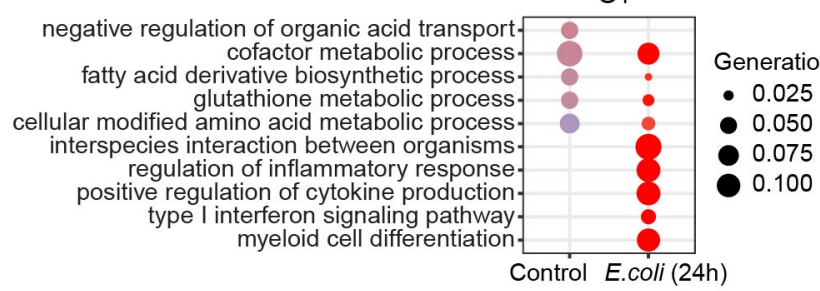

$\mathrm{G} 5 \mathrm{~b}$

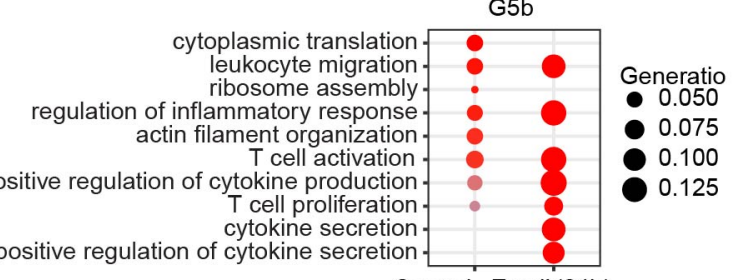

Control E.coli (24h)

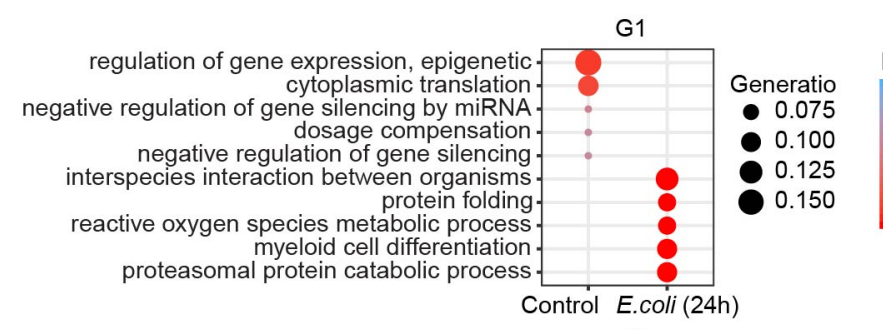

G3

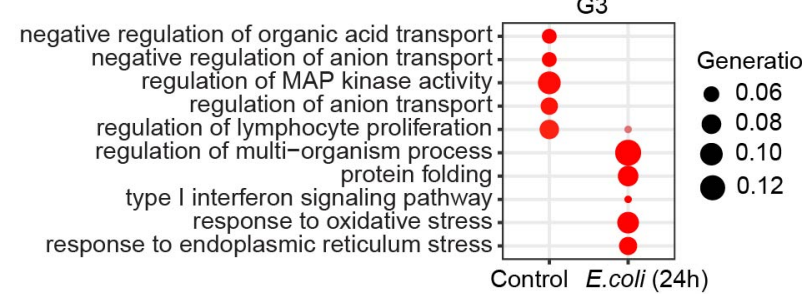

Control E.coli (24h)

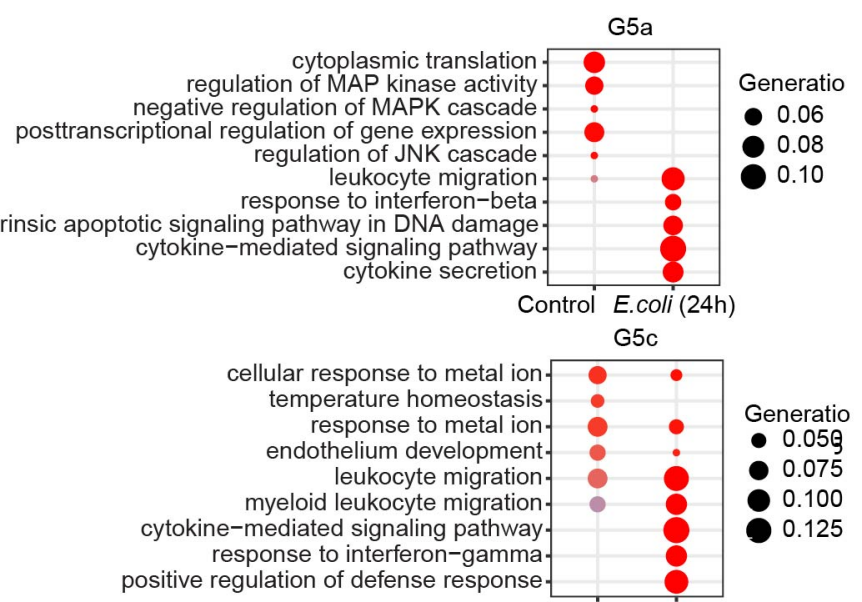

p.adjust 0.05

0.04

0.03

0.02

0.01 
Figure S5. Differentially expressed genes in each neutrophil subpopulation in $E$. coli-challenged mice.

(A) MA plots displaying genes that are up- (red) or downregulated (blue) after E. coli challenge for each cluster. Dashed lines denote fold change thresholds used when identifying DEGs.

(B) Gene ontology (GO) analysis of DEGs before and after E. coli challenge for each cluster. Selected GO terms with Benjamini-Hochberg-corrected P-values $<0.05$ (one-sided Fisher's exact test) are shown. 
A
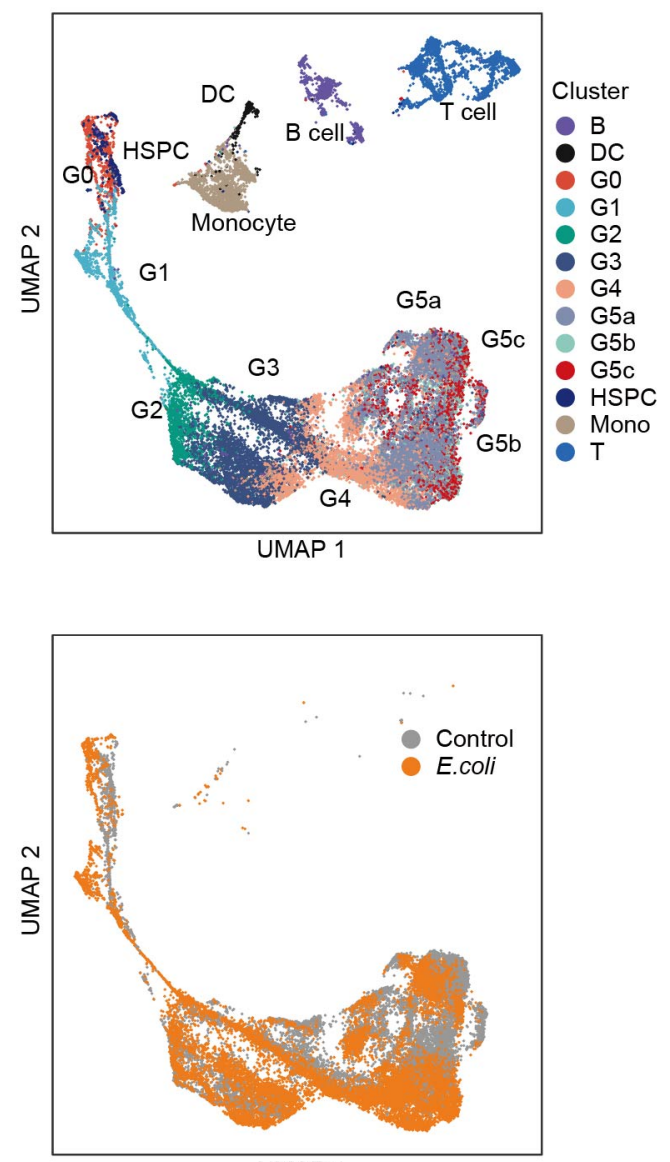

UMAP 1

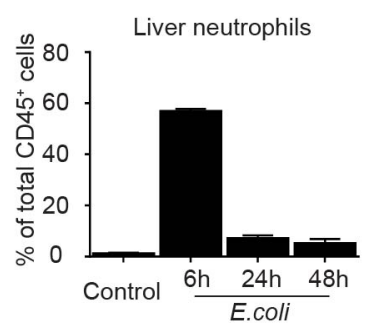

F
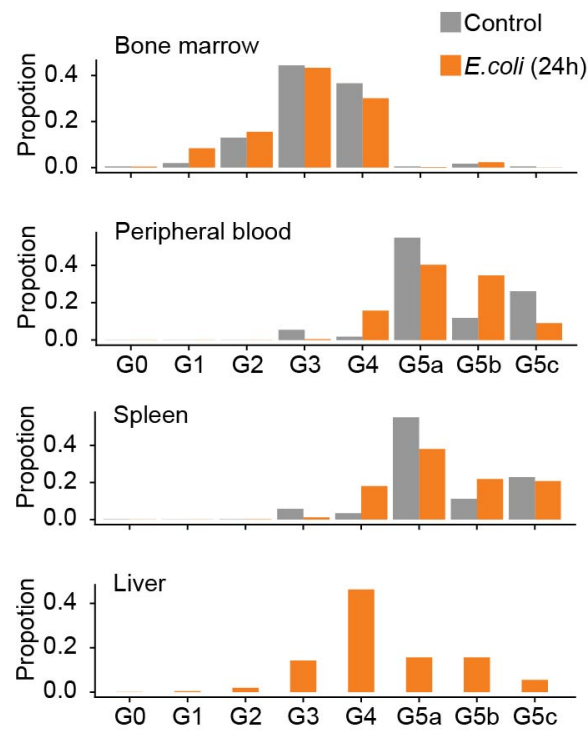

B

Regulon activity

(t-value E.coli challenged versus control in each group)

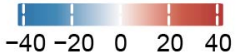

Cebpe (570 genes) Ets 1 (600 genes) $\mathrm{Nfe} 2$ (352 genes Ltf (123 genes) KIf5 (225 genes) Cebpb (1152 genes) fill 318 genes Irf9 (399 genes) Rara (188 genes) Irf5 (472 genes) Nr1h3 (32 genes) Hdac1 13 genes Snai1 (19 genes) Klf4 (119 genes) Mxd1 24 genes $\mathrm{Bcl} 3$ (307 genes) Bi1 (11 Irt7 822 genes Meis1 (166 genes) os (1010 genes Er1 121 genes Aun 838 genes Jund (838 genes Jun $(829$ genes Atf (207 genes Att3 (207 genes) Cebpa (110 genes Hoxa9 88 genes Ezh2 (2507 genes) Smarca4 (1252 genes) Ctcf (1478 genes) Sin3a 297 genes oxn3 (158 genes Etv6 315 genes Nrf1 106 genes Foxp1 (377 genes Trim28 (365 genes) Taf1 (496 genes) Kdm5a (640 genes) Elk4 (208 genes) Fli1 606 genes Mxi1 329 genes Gtf2f1 672 genes Usf2 (555 genes, Hcfc1 1659 genes Srebf1 $(80$ genes) Atf1 (349 genes) Brf1 (290 genes) Smarcb1 (168 genes) Sp1 (218 genes)

$E$

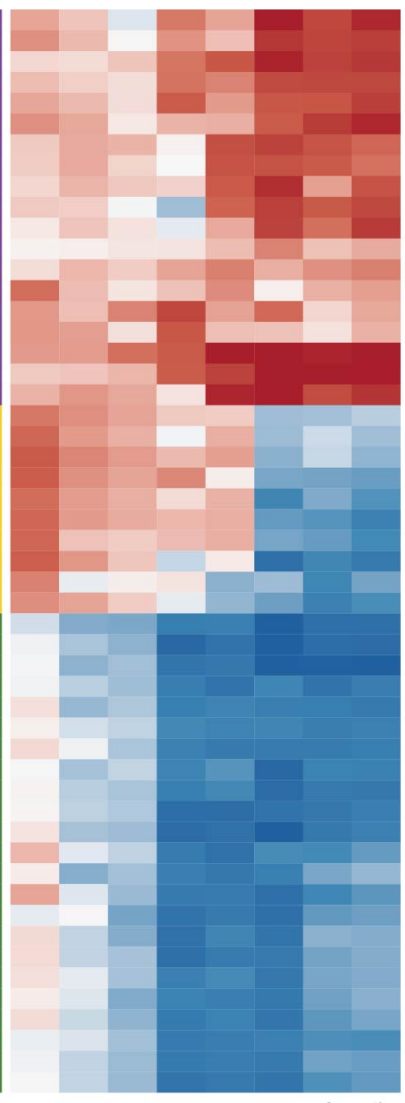

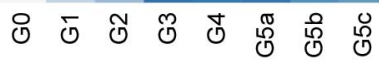

C Regulon activity (t-value versus previous stage under normal condition

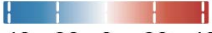
$-40-20 \quad 0 \quad 20 \quad 40$

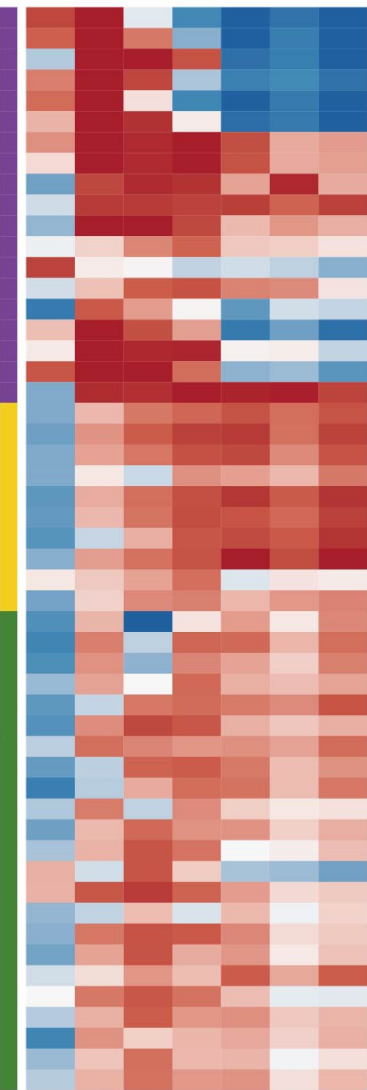

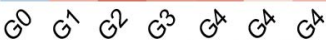

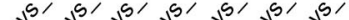
$\mathrm{O}^{\prime \prime} \mathrm{O}^{2} \mathrm{O}^{3} \mathrm{O}^{\alpha} \mathrm{G}^{\mathrm{S}^{2}} \mathrm{O}^{\circ} \mathrm{O}^{\circ}$
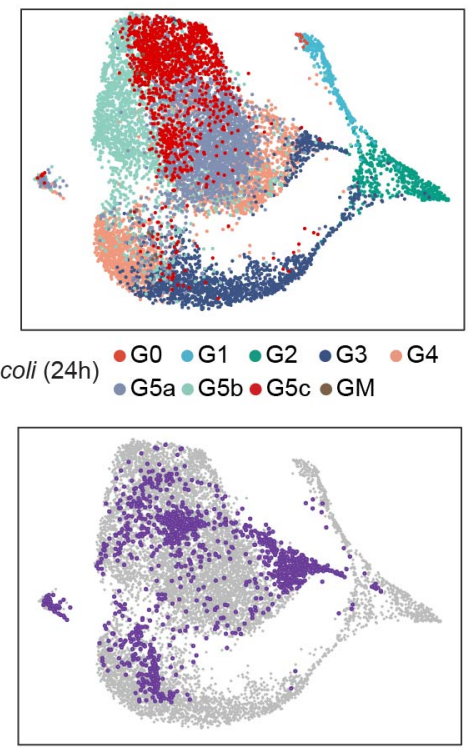

- Liver(E.coli 24h) Others

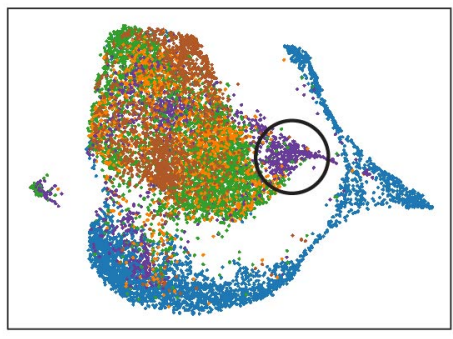

- $\mathrm{BM} \bullet \mathrm{PB} \bullet \mathrm{SP} \bullet \mathrm{LV} \bullet \mathrm{PC}$

Liver

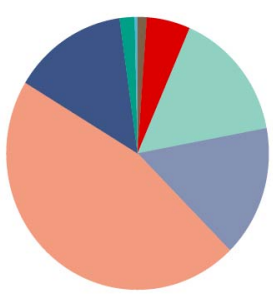




\section{Figure S6.}

\section{(A-C) Alteration of transcription networks in E. coli-challenged mice.}

(A) UMAP of the regulon activity matrix of 32,888 cells (11,992 normal neutrophils, 13,687 challenged neutrophils, and 7209 other cells under normal conditions) colored by Seurat cluster identity (left) or experimental condition (right, only neutrophils).

(B) Heatmap of the t-values of regulon activity derived from a generalized linear model for the difference between cells from one challenged neutrophil subpopulation and cells from the corresponding normal subpopulation. Only regulons with at least one absolute t-value greater than 18 are visualized. Regulons are hierarchically clustered based on challenge-response pattern (purple: upregulated, yellow: first up- then downregulated, green: downregulated)

(C) Heatmap showing activity change of regulons identified in (B) during normal group transitions.

(D-F) The liver displays a distinct extramedullary granulopoiesis program during bacterial infection.

(D) Neutrophil proportions in the liver measured at different time points after E. coli challenge. Results are the mean $( \pm \mathrm{SD})$ of three independent experiments.

(E) Composition of liver neutrophils after E. coli challenge. Top: UMAP of E. coli-challenged neutrophils from BM, PB, SP, liver (LV), and peritoneal cavity (PC) colored by cluster identity. Bottom: Liver cells are highlighted in the UMAP plot. Composition of the liver neutrophils after E. coli challenge is displayed on the right.

(F) Comparisons of organ distribution of each neutrophil cluster before and after E. coli challenge. 
A
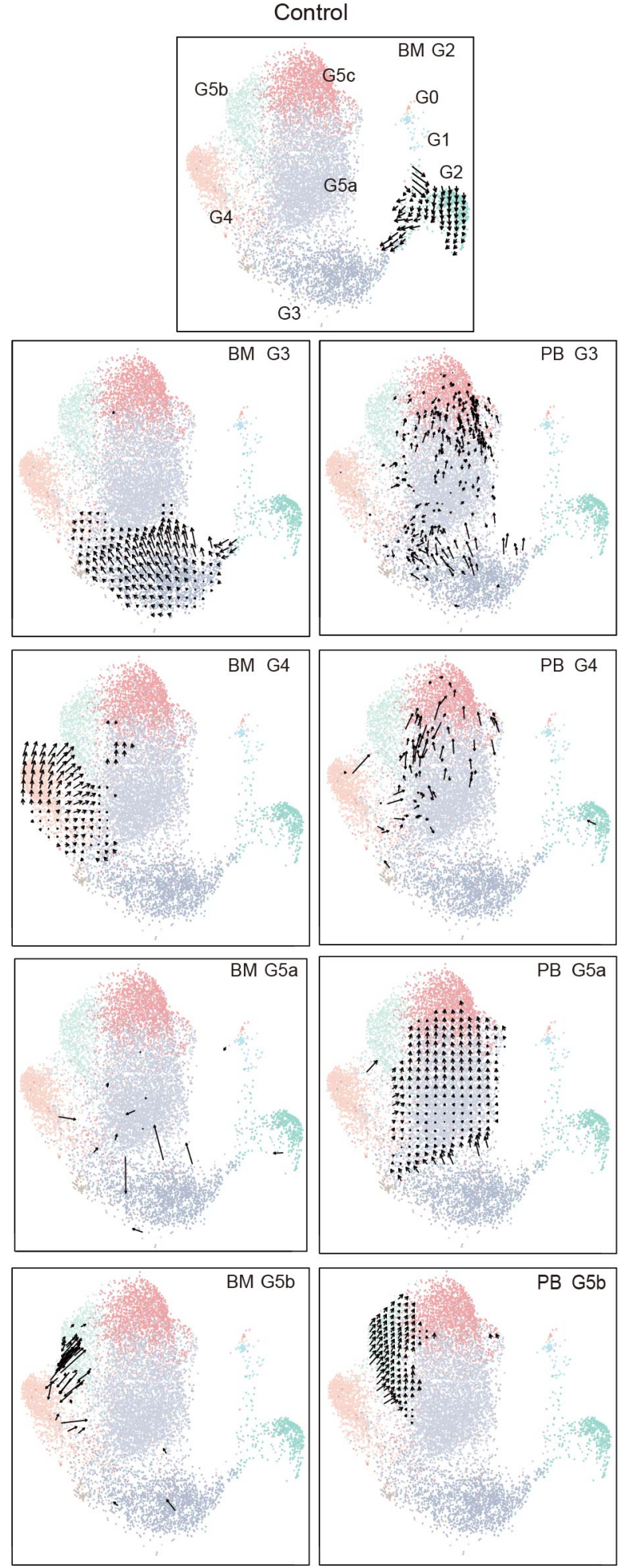

BM G5c is,
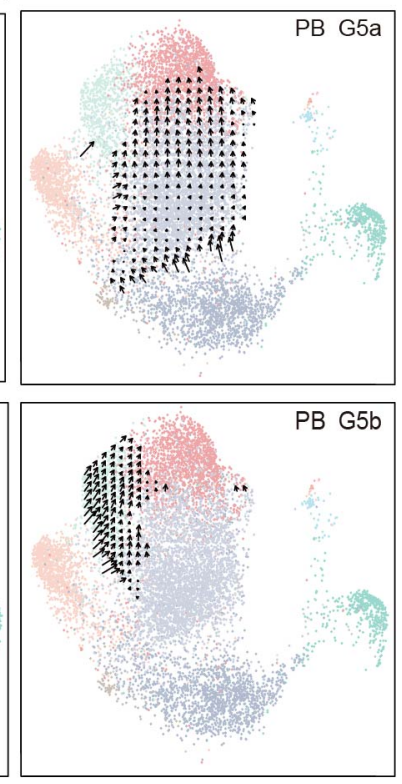

B
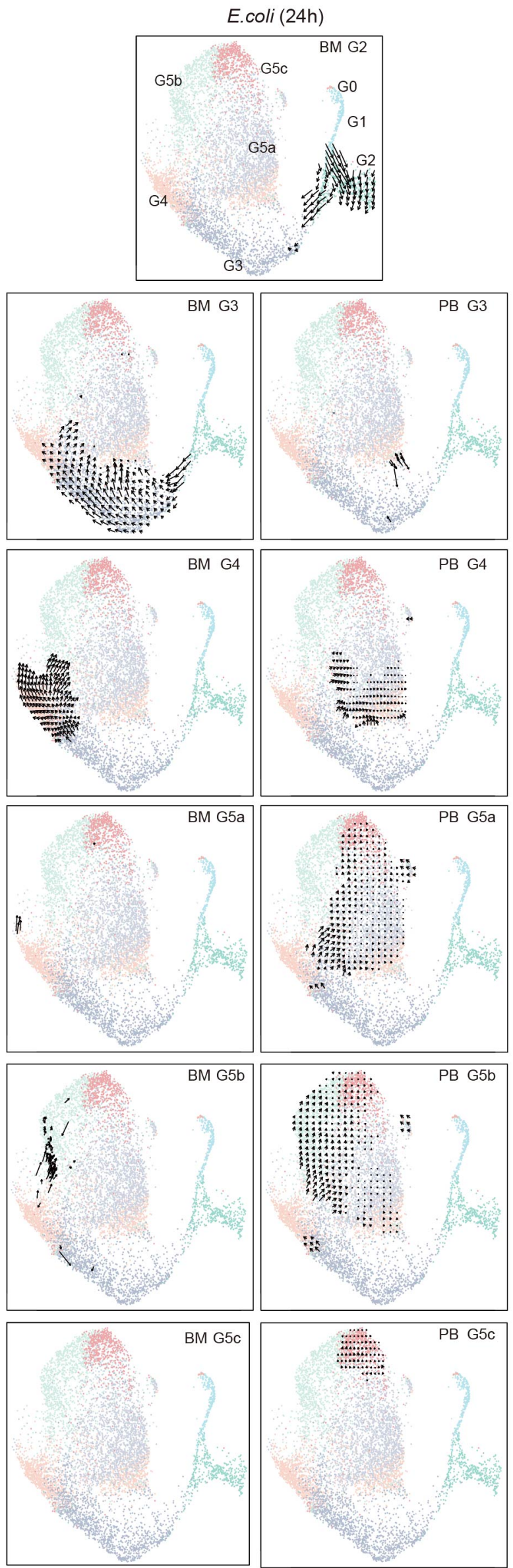
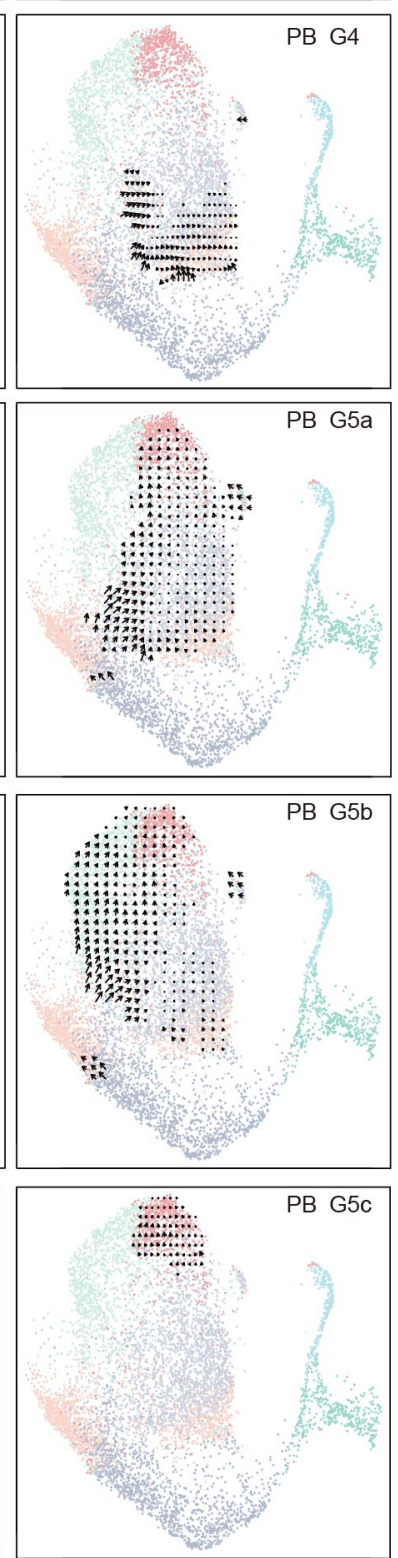
Figure S7. Neutrophil dynamics under steady-state and bacterial infection conditions assessed by velocity analysis.

(A) Dynamics (velocity field projected on the UMAP plot) of G2-G5 neutrophils under normal conditions. BM neutrophils (left) and PB neutrophils (right) are displayed separately. For small populations, velocity vectors of all cells are visualized directly. For large populations, a grid velocity summary is derived by calculating the Gaussian-weighted average of velocity vectors of all cells at each grid point. Only grid points adjacent to neutrophils in the target population are visualized.

(B) As in (A) but of challenged neutrophils. 